\title{
Dimension Vectors of Indecomposable Objects for Nilpotent Operators of Degree 6 with One Invariant Subspace
}

\author{
Piotr Dowbor ${ }^{1} \cdot$ Hagen Meltzer ${ }^{2}$
}

Received: 8 October 2016 / Accepted: 13 December 2017 / Published online: 10 February 2018

(C) Springer Science+Business Media B.V., part of Springer Nature 2018

\begin{abstract}
Formulas for the dimension vectors of all objects $M$ in the category $\mathcal{S}(\tilde{6})$ of nilpotent operators with nilpotency degree bounded by 6 , acting on finite dimensional vector spaces with invariant subspaces in a graded sense, are given (Theorem 2.3). For this purpose we realize a tubular algebra $\Lambda$, controlling the category $\mathcal{S}(\tilde{6})$, as an endomorphism algebra of a suitable tilting bundle over a weighted projective line of type $(2,3,6)$ (Theorem 3.6). Using this description and a concept of mono-epi type, the interval multiplicity vector of an object in $\mathcal{S}(\tilde{6})$ is introduced and determined (Theorem 2.8). This is a much finer invariant than the usual dimension vector.
\end{abstract}

Keywords Nilpotent operator · Invariant subspace · Submodule category · Tubular algebra $\cdot$ Dimension vector $\cdot$ Interval multiplicity vector $\cdot$ Exceptional object $\cdot$ Weighted projective line $\cdot$ Coherent sheaf $\cdot$ Tilting bundle $\cdot$ Telescoping functor $\cdot$ Mono-epi representation $\cdot$ Mono-epi-type

Mathematics Subject Classification (2010) $16 \mathrm{G} 30 \cdot 16 \mathrm{G} 20 \cdot 14 \mathrm{~F} 05 \cdot 47 \mathrm{~A} 15 \cdot 15 \mathrm{~A} 04 \cdot$ $16 \mathrm{D} 70 \cdot 16 \mathrm{G} 70$

Dedicated to Professor Daniel Simson on the occasion of his 75th birthday

Presented by Henning Krause.

Piotr Dowbor

dowbor@mat.umk.pl

Hagen Meltzer

hagen.meltzer@usz.edu.pl

1 Faculty of Mathematics and Computer Science, Nicolaus Copernicus University, Chopina 12/18, 87-100 Toruń, Poland

2 Institute of Mathematics, Szczecin University, Wielkopolska 15, 70-451 Szczecin, Poland 


\section{Introduction}

In 1934 G. Birkhoff investigated the combinatorial structure of $p$-groups and their subgroups. More precisely he studied the category $\mathcal{S}(A)$ which objects are pairs $\left(Z, Z^{\prime}\right)$, where $Z$ is a finitely generated module over $\mathbb{Z}_{p^{n}}$ and $Z^{\prime}$ is a submodule of $Z$. For $n \leq 5$ he was able to solve the problem completely, in contrast to the case $n=6$, which is still waiting for a proper treatment.

The problem becomes even more interesting if we replace $\mathbb{Z}_{p^{n}}$ by other uniserial rings, in particular by the algebras $A=k[x] /\left(x^{n}\right)$, where $k$ is a field. Then the submodule category $\mathcal{S}(A)$, which will be further called $\mathcal{S}(n)$, is just the category of finite-dimensional vector spaces over $k$ endowed with nilpotent operators of nilpotency degree bounded by $n$ and invariant subspaces.

The categories $\mathcal{S}(n)$ were studied quite intensively recently. D. Simson determined the representation type of $\mathcal{S}(n)$, dependently on $n$ [25]. In fact, he solved the problem of the representation type not only for submodule categories, but more generally, for the categories of chains of submodules of arbitrary lengths. His solution, to some extend, is similar to that in the original situation. More precisely, if $n \leq 5$ (respectively, $n \geq 7$ ) the submodule category $\mathcal{S}(n)$ is representation finite (respectively, wild) and for $n=6$ it is tame (see also [22]). The further essential progress concerning the categories $\mathcal{S}(n)$ was done by C. M. Ringel and M. Schmidmeier in [23] (see also [21, 22, 24]). They described in detail the structure of $\mathcal{S}(n)$ in the most interesting case $n=6$, showing that the Auslander-Reiten quiver of $\mathcal{S}(6)$ consists only of $\mathbb{P}^{1}(k)$-families of tubes.

A basic role in understanding the structure of the $\mathcal{S}(n)$ 's, in [23] is played by the categories $\mathcal{S}(\tilde{n})$, which are the $\mathbb{Z}$-graded variants of the $\mathcal{S}(n)$ 's. It occurs that the natural covering functors $F_{n}: \mathcal{S}(\tilde{n}) \rightarrow \mathcal{S}(n)$, defined by forgetting the grading, have nice properties, in particular, they are dense provided $n \leq 6$. An elegant description of the structure of $\mathcal{S}(6)$ is based just on this fact and refers to the shape of a fundamental domain $\mathcal{D}$ in $\mathcal{S}(\tilde{6})$ (more precisely in its Auslander-Reiten quiver) with respect to the action of the group $\mathbb{Z}$ given by shift of the grading. $\mathcal{D}$ is a disjoint union of $\mathbb{P}^{1}(k)$-families of tubes each of them of tubular type $(2,3,6)$. It is proved that the functor $F_{6}$ yields a bijection between the sets of isoclasses of all objects from $\mathcal{D}$ and of isoclasses of all indecomposable objects from $\mathcal{S}(6)$.

Using completely different methods in [10] it was shown that the stable category of $\mathcal{S}(\tilde{n})$ is equivalent to the stable category of vector bundles over a weighted projective line of weight type $(2,3, n)$ in the sense of [7]. Here the notion of a stable category of vector bundles means that in the category of vector bundles we factor out the ideal of all morphisms which factor through finite direct sums of line bundles. The investigation of such categories and their connections to categories of nilpotent operators with chains of invariant submodules was continued in [11] and [12].

The main aim of this paper is to determine the dimension vectors of the indecomposable objects in $\mathcal{S}(\tilde{6})$, with a particular focus on the exceptional ones. We consider these objects as representations over a certain algebra $R$ given by a quiver with relations (for the precise definition of $R$ see Section 2.1). In fact, $R$ is the universal cover of the $2 \times 2$ triangular matrix algebra $T_{2}\left(k[x] /\left(x^{6}\right)\right)$ and it admits a natural action of the group $\mathbb{Z}$ such that the induced action on the category $\bmod (R)$ of finite dimensional right $R$-modules by restriction to $\mathcal{S}(\tilde{6})$ coincides with the $\mathbb{Z}$-action mentioned before. Notice that in contrast to objects belonging to homogeneous tubes (all of them clearly admit non-trivial selfextensions), 
the general problem of determining the dimension vectors was not solved in [23]. Recall that an object $X$ in an abelian $k$-category is called exceptional if $\operatorname{End}(X)$ is a skew field (in case $k$ is algebraically closed this means that $\operatorname{End}(X)=k$, if $\operatorname{End}(X)$ is finite dimensional) and $\operatorname{Ext}^{n}(X, X)=0$, for all $n \geq 1$. By general facts the exceptional objects are uniquely determined by their dimension vectors, so their knowledge is useful. The description of these dimension vectors, given in Theorem 2.3, is expressed in terms of certain matrices which are basically obtained from the tubular mutations in the sense of $[14,16,17]$. These functors were introduced there in order to classify the indecomposable sheaves over a tubular weighted projective line.

A crucial point in the proof of our result is played by an explicit realization of a certain tubular algebra $\Lambda$ which was introduced in [23]. This algebra is a subcategory of $R$, if both, $\Lambda$ and $R$, are regarded as $k$-categories; hence, all modules over $\Lambda$ can be treated as modules over $R$. Moreover, $\Lambda$ has the property that all indecomposable objects of the fundamental domain $\mathcal{D}$ for $\mathcal{S}(\tilde{6})$, with the exception of those in a certain rank-6 tube, under the canonical restriction belong to the category $\bmod (\Lambda)$ of finite dimensional right $\Lambda$-modules. We find a concrete tilting object $T$ in the category $\operatorname{coh}(\mathbb{X})$ of coherent sheaves over a weighted projective line $\mathbb{X}$ of type $(2,3,6)$ such that its endomorphism algebra $\operatorname{End}(T)$ is isomorphic to $\Lambda$. The tilting sheaf $T$ is given as a direct sum of some vector bundles of rank smaller than or equal to 6 and the proof of the realization above is mainly K-theoretical. Then using the equivalence of the bounded derived categories $\mathcal{D}^{b} \operatorname{coh}(\mathbb{X}) \simeq \mathcal{D}^{b} \bmod (\Lambda)$ we can adopt the techniques of tubular mutations and telescopic functors in a similar way as in the case of canonical algebras of tubular type $[3,4,17,18]$. We stress the fact that the proposed method of finding a tilting object in the category $\operatorname{coh}(\mathbb{X})$ is quite general and can be used for tilting realizations for other concealed-canonical algebras. We intend to study further tubular cases related to problems of chains of submodules in a forthcoming paper.

As a byproduct of our tilting theory approach we obtain that for all indecomposable objects in $\mathcal{S}(\tilde{6})$, except of the members of some very special tubes, the linear structure maps are monomorphisms or epimorphisms. This is an important result which is used in further considerations.

In this paper we will also study the interval multiplicities for the indecomposable $\Lambda$ modules and objects of $\mathcal{S}(\tilde{6})$. Recall that an indecomposable module over a linear quiver is given by an interval of the vertex set where the chosen vector spaces for all points of this interval are $k$ and the chosen linear maps for the arrows are identities. We use this for a convenient description of the finite dimensional modules over the algebra $\Lambda$ and the related algebra $R$. Note that $R$ treated as a $k$-category contains two disjoint copies of a category $\tilde{A}$, whose ordinary quiver is the infinite equioriented linear quiver with vertex set $\mathbb{Z}$, such that each object of $R$ belongs to some of them. Applying these ideas we will in 2.7 define for a module $M$ in $\bmod (R)$ the interval multiplicity vector $m(M)$, which is a much finer invariant than the dimension vector of $M$ and it is useful in order to describe the possibly simplest matrix representation for $M$.

The second main result of this paper is the determination of the interval multiplicities for indecomposable objects in $\mathcal{S}(\tilde{6})$ (Theorem 2.8). In order to do so we introduce the concept of abstract mono-epi types and representations belonging to them. We study these notions for uniserial path algebras and their quotients, and finally for the algebra $\Lambda$. Moreover, we give also a description of so called strict types in terms of the sections in the AuslanderReiten quivers of the considered algebras and recognize the interval decompositions in case of the two distinguished types arising by means of our tilting procedure. 
The results above will be used in a forthcoming joint paper with M. Schmidmeier [5] in order to show that all exceptional objects in $\mathcal{S}(\tilde{6})$ can be exhibited by matrices having as coefficients only 0 and 1, a result which is related to work of P. Gabriel on Dynkin quivers [6], C. M. Ringel on path algebras of finite quivers without oriented cycles [20] and also our work on canonical algebras [13, 18] and [4].

The paper is organized as follows. In Section 2 we introduce the notions and notations, which are necessary to present our main results, Theorem 2.3 and Theorem 2.8 . There also some facts following from these theorems are formulated (Corollaries 2.5 and 2.9). Section 3 is devoted to the proof of Theorem 3.6 on the realization of the tubular algebra $\Lambda$ as an endomorphism algebra of a concrete tilting sheaf over a weighted projective line $\mathbb{X}$. In particular, we show that $\Lambda$ is isomorphic to the endomorphism algebra of a tilting bundle with the prescribed rank vector, if these two algebras have the same Cartan matrices (Proposition 3.9). In Section 4, the comparison result (Theorem 4.1) and its consequence (Proposition 4.6), which yields a description of the quasi-simple exceptional objects from $\mathcal{D}$ in terms of sheaves, are formulated. We also prove Theorem 2.3, applying the previous result, and perform a detail discussion of the mono-epi property for indecomposable $\Lambda$-modules with suitable slopes (Propositions 4.10 and 4.12, Corollary 4.14). Section 5 contains a systematic survey of basic facts concerning the notion of mono-epi types for the case of uniserial path algebras and their factors (Lemma 5.3, Proposition 5.4), which ends up with the classification result (Theorem 5.7). They are adopted to the context of $\tilde{A}$ (Proposition 5.9) and next applied in the situation of some special types (Proposition 5.11). The considerations of this part of the paper are completed by the proof of Theorem 2.8. Section 6 is devoted to some examples, illustrating how to apply our two results in practice for selected values of the index.

We would like to thank very much Markus Schmidmeier for pointing out, during joint discussions on the "0,1-property" for objects of $\mathcal{S}(\tilde{6})$ in May 2015 in Torun, that some elements of our project he has developed independently earlier with Claus M. Ringel (e.g. some elements of Corollary 4.14), and for convincing us about the importance of the interval decompositions in our considerations. Unfortunately, up to our knowledge, the precise formulations of the results by Ringel and Schmidmeier (and their proofs) are not available in any form, which make them possible to quote.

In the paper we use standard definitions and notation, which are well known and commonly used. For example, we denote by $\mathbb{N}$ (respectively, by $\mathbb{N}_{m}$ ) the set of all natural numbers with 0 (respectively, greater than or equal to $m$ ). We use the similar notation in case of the sets $\mathbb{Z}$ and $\mathbb{Q}$ of all integers and rational numbers, respectively. For any $n \in \mathbb{N}_{1}$ (respectively, $m, n \in \mathbb{N}_{1}$ such that $m \leq n$ ) we set $[n]:=\{1, \ldots, n\}$ (respectively, $[m, n]:=\{m, \ldots, n\})$. If $A$ is a commutative ring then by $A^{m}$ we always mean the free $A$-module of rank $m$, consisting of the column vectors of the respective size. For a matrix $P \in \mathbb{M}_{m \times n}(A)$, by $P^{\langle j\rangle}$ we denote the vector in $A^{m}$ being the $j$ th column of $P$, where $j \in[m]$; moreover, we set $P^{\langle i, j\rangle}:=P^{\langle i\rangle}+\ldots+P^{\langle j\rangle}$, if $i \leq j$. (Clearly, $\left.P^{\langle i, i\rangle}=P^{\langle i\rangle}\right)$.

For basic information concerning modules and representation theory of algebras (respectively, derived categories of module categories) we refer to [1] (respectively [8]). The most important specialized notions, for the benefit of the reader will be briefly recalled in the next consecutive sections. All fields used in the paper for simplicity are assumed to be algebraically closed. 
The authors would like to thank the referee for the comments and suggestions, which considerably contributed to improvements in the presentation of the results.

\section{The main results}

We start by fixing the notations which are necessary to formulate our results.

\section{1}

Let $(\tilde{Q}, \tilde{\Delta})$ be an infinite bounded quiver, where $\tilde{Q}$ is given below

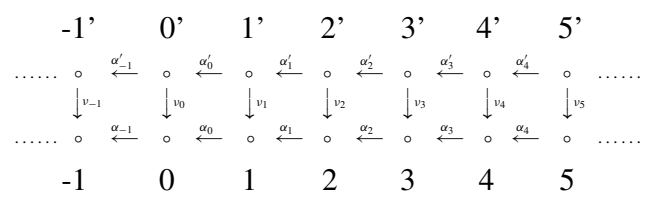

and $\tilde{\Delta}=\left\langle\alpha_{i+5} \ldots \alpha_{i}, \alpha_{i+5}^{\prime} \ldots \alpha_{i}^{\prime}, \alpha_{i}^{\prime} v_{i}-v_{i+1} \alpha_{i}: i \in \mathbb{Z}\right\rangle$. It is clear that the category $\tilde{\mathcal{S}}:=\mathcal{S}(\tilde{6})$ in a natural way can be regarded as a full subcategory of the category $\tilde{\mathcal{H}}:=\bmod (R)=\operatorname{rep}_{k}(\tilde{Q}, \tilde{\Delta})$ of finite dimensional right modules over the locally bounded category $R$ associated to $(\tilde{Q}, \tilde{\Delta})$ (equivalently, all finite dimensional representations of $(\tilde{Q}, \tilde{\Delta}))$, consisting of all these $M=\left(M_{v}, M_{\alpha}\right)_{v \in \tilde{Q}_{0}, \alpha \in \tilde{Q}_{1}}$ for which $M_{v_{j}}$ is a monomorphism for every $j \in \mathbb{Z}$. The category $R$ is equipped with a natural action of the group $\mathbb{Z}$ such that the induced action $(z, M) \mapsto{ }^{z} M$ on $\tilde{\mathcal{H}}$ (and $\tilde{\mathcal{S}}$ ) is given by "shifting of $M$ by $z$ to the right", which means that $\left({ }^{z} M\right)_{n}=M_{n-z}$ and $\left({ }^{z} M\right)_{n^{\prime}}=M_{(n-z)^{\prime}}$, for any $n \in \mathbb{Z}$. (By some technical reasons, in the definition of $\tilde{Q}$ we use here another notation as in [23]; e.g. the indexing of vertices of $\tilde{Q}$ is such that $s\left(\alpha_{n}\right)=n+1$ and $\left.h\left(\alpha_{n}\right)=n\right)$.

Recall that the description of the structure of $\tilde{\mathcal{S}}$ from [23], in particular, of the indecomposable objects and the Auslander-Reiten components, is fully given (up to the $\mathbb{Z}$-shift) by a fundamental domain $\mathcal{D}$ being a disjoint union of $\mathbb{P}^{1}(k)$ - families $\mathcal{T}_{\gamma}$ of tubes in $\tilde{\mathcal{S}}$, for $\gamma \in \mathbb{Q}_{0}=\{q \in \mathbb{Q}: q \geq 0\}$, each of tubular type $(2,3,6)$. (Note that this fundamental domains differs slightly from the original one). All the families but $\mathcal{T}_{0}$ consist of regular tubes, in $\mathcal{T}_{0}$ only the rank-6 tube is not regular. The shifts ${ }^{m} \mathcal{D}$, for various $m \in \mathbb{Z}$, are pairwise disjoint and the Auslander-Reiten quiver of the category $\tilde{\mathcal{S}}$ is equal to the union $\bigcup_{m \in \mathbb{Z}}{ }^{m} \mathcal{D}$. If necessary, we treat further $\mathcal{D}$ also as a corresponding class of indecomposable objects in $\tilde{\mathcal{S}}$ which is closed under isomorphisms.

We fix the following notation. For any $\gamma \in \mathbb{Q}_{0}$ and $i \in[3]$ such that $(\gamma, i) \neq(0,3)$, we denote by $M_{i, 0}(\gamma), \ldots, M_{i, p_{i}-1}(\gamma)$ fixed representatives of the consecutive (in the sense of Auslander-Reiten translation $\tau^{-}$) isoclasses of quasi-simple objects from the mouth in the exceptional tube of rank $p_{i}$ in the family $\mathcal{T}_{\gamma}$, where $p_{1}=2, p_{2}=3$ and $p_{3}=6$. (In fact, the indexing of quasi-simples in the tubes is provided in some canonical coherent way, which is independent on $\gamma$, see 4.7). Moreover, for any $l \in \mathbb{N}_{1}$, we denote by $M_{i, s, l}(\gamma)$ a fixed object from the tube of rank $p_{i}$ in $\mathcal{T}_{\gamma}$, determined uniquely up to isomorphism, which is of quasi-length $l$ and has quasi-socle isomorphic to $M_{i, s}(\gamma)$, where $s \in \mathbb{Z}_{p_{i}}$ (clearly, we can assume $M_{i, s, 1}(\gamma)=M_{i, s}(\gamma)$ ). We give explicit formulas determining directly the dimension vectors $\underline{\operatorname{dim}_{k}} M_{i, s}(\gamma)$ (respectively, $\underline{\operatorname{dim}_{k}} M_{i, s, l}(\gamma)$ ). 


\section{2}

Let $\dot{S}, \dot{R}, U \in \mathbb{M}_{10}(\mathbb{Z})$ be the following triple of invertible (over $\mathbb{Z}$ ) matrices given below

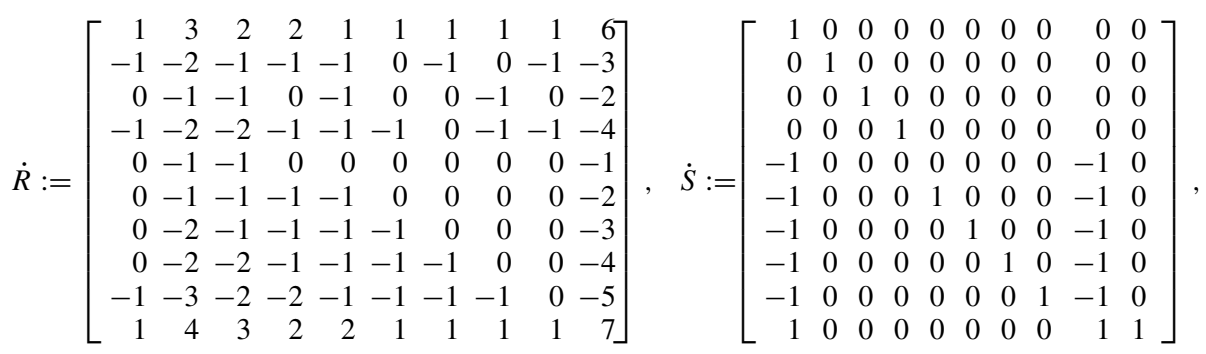

$$
U:=\left[\begin{array}{rrrrrrrrrr}
0 & 0 & 1 & 0 & 0 & 0 & 0 & 1 & 0 & 1 \\
0 & 1 & 1 & 1 & 1 & 0 & 0 & 1 & 1 & 3 \\
-1 & 2 & 2 & 1 & 1 & 1 & 0 & 1 & 1 & 5 \\
-1 & 2 & 2 & 2 & 1 & 1 & 1 & 1 & 1 & 6 \\
-1 & 2 & 2 & 1 & 1 & 0 & 1 & 1 & 1 & 5 \\
-1 & 1 & 1 & 1 & 1 & 0 & 0 & 1 & 0 & 3 \\
-1 & 0 & 0 & 0 & 1 & 0 & 0 & 0 & 0 & 1 \\
0 & 2 & 2 & 1 & 1 & 1 & 0 & 1 & 1 & 4 \\
0 & 1 & 1 & 1 & 0 & 1 & 0 & 1 & 1 & 3 \\
0 & 0 & 1 & 0 & 0 & 0 & 0 & 0 & 1 & 1
\end{array}\right]
$$

In fact, these matrices correspond to some important operations on the Grothendieck group level, their actual meaning will be explained in Lemma 3.11 and Corollary 4.2. To any $\gamma=$ $\frac{\gamma_{\infty}}{\gamma_{0}} \in \mathbb{Q}_{0}$, with $\gamma_{\infty} \in \mathbb{N}, \gamma_{0} \in \mathbb{N}_{1}$ being coprime, we associate the matrix $\Xi_{\gamma} \in \mathbb{M}_{10}(\mathbb{Z})$, defined as follows:

$$
\Xi_{\gamma}:=\left\{\begin{array}{cl}
U & \text { if } \gamma=1, \\
\epsilon_{\gamma} U \cdot \dot{S}^{c_{1}} \cdot \dot{R}^{c_{2}} \cdot \dot{S}^{c_{3}} \cdot \ldots \cdot \dot{S}^{c_{m-1}} \cdot \dot{R}^{c_{m}} & \text { if } \gamma \neq 1 \text { and } m \in 2 \mathbb{N}, \\
\epsilon_{\gamma} U \cdot \dot{S}^{c_{1}} \cdot \dot{R}^{c_{2}} \cdot \ldots \cdot \dot{R}^{c_{m-1}} \cdot \dot{S}^{c_{m}-1} \cdot \dot{R} & \text { if } \gamma \neq 1 \text { and } m \notin 2 \mathbb{N},
\end{array}\right.
$$

where $c(q)=\left[c_{1} ; c_{2}, \ldots, c_{m}\right]$ is the continued fraction presentation of the rational number $q \in \mathbb{Q}$, for $q=q(\gamma):=\frac{\gamma_{0}}{\gamma_{0}-\gamma_{\infty}}$, and $\epsilon_{\gamma}:=1$, if $\gamma \leq 1$ (respectively, $\epsilon_{\gamma}:=-1$, if $\gamma>1$ ). Notice that the mapping $\gamma \mapsto q(\gamma)$ yields an injection $\mathbb{Q}_{0} \longrightarrow \overline{\mathbb{Q}}:=\mathbb{Q} \cup\{\infty\}$ such that $q(1)=\infty$.

For any vector $d=\left[d_{1}, \ldots, d_{10}\right]^{t r} \in \mathbb{Z}^{10}$ we denote by $\xi(d)=\left[\begin{array}{l}\left(\hat{d}_{z^{\prime}}\right)_{z \in \mathbb{Z}} \\ \left(\hat{d}_{z}\right)_{z \in \mathbb{Z}}\end{array}\right]$ the associated dimension vector over the category $R$, of the shape

$$
\xi(d):=\left[\begin{array}{llllllllllll}
\cdots & 0 & 0 & d_{1} & d_{2} & d_{8} & d_{9} & d_{10} & 0 & 0 & 0 & \ldots \\
\cdots & 0 & 0 & d_{1} & d_{2} & d_{3} & d_{4} & d_{5} & d_{6} & d_{7} & 0 & \ldots
\end{array}\right]
$$

where $\hat{d}_{1}=d_{1}=\hat{d}_{1^{\prime}}$.

Now we can present our main result. 
Theorem 2.3 Let $(\gamma, i, s)$ be an arbitrary triple such that $\gamma \in \mathbb{Q}_{0}, i \in[3]$ and $s \in \mathbb{Z}_{p_{i}}$, with $(\gamma, i) \neq(0,3)$. Then the dimension vector of the quasi-simple object $M_{i, s}(\gamma)$ in $\tilde{\mathcal{S}} \subseteq \tilde{\mathcal{H}}$ is given as follows:

$$
(* *) \quad \operatorname{dim}_{k} M_{i, s}(\gamma)=\left\{\begin{array}{lll}
\xi\left(\Xi^{\langle 10\rangle}-\Xi^{\langle 2\rangle}\right) & \text { if } i=1, s=0, \\
\xi\left(\Xi^{\langle 2\rangle}\right) & \text { if } i=1, s=1, \\
\xi\left(\Xi^{\langle 10\rangle}-\Xi^{\langle 3,4\rangle}\right) & \text { if } i=2, s=0, \\
\xi\left(\Xi^{\langle s+2\rangle}\right) & \text { if } i=2, s=1,2, \\
\xi\left(\Xi^{\langle 10\rangle}-\Xi^{\langle 5,9\rangle}\right) & \text { if } i=3, s=0, \\
\xi\left(\Xi^{\langle s+4\rangle}\right) & \text { if } i=3, s=1, \ldots, 5 .
\end{array}\right.
$$

where $\Xi=\Xi_{\gamma} \in \mathbb{M}_{10}(\mathbb{Z})$.

The proof of the theorem needs longer preparations and will be given in Section 4.7.

\section{4}

To formulate the precise description of the dimension vectors for all indecomposable objects in $\tilde{\mathcal{S}}$ we denote by $\kappa_{i, s, 1}(\gamma)$ the expressions on the right hand side of the formula $(* *)$, for the respective triples $(\gamma, i, s)$. Moreover, for any $\gamma \in \mathbb{Q}_{0}$ we set

$$
\kappa_{2,0,2}(\gamma):=\xi\left(\Xi^{\langle 10\rangle}-\Xi^{\langle 4\rangle}\right), \kappa_{2,1,2}(\gamma):=\xi\left(\Xi^{\langle 3,4\rangle}\right), \kappa_{2,2,2}(\gamma):=\xi\left(\Xi^{\langle 10\rangle}-\Xi^{\langle 3\rangle}\right),
$$

additionally, for any $s \in \mathbb{Z}_{6}$ and $2 \leq r \leq 5$, with $(\gamma, s) \neq(0,3)$, we denote by $\kappa_{3, s, r}(\gamma)$ the expression in the $r$ th row and $s$ th column of the table below (by technical reasons it is splitted into two parts)

\begin{tabular}{llll}
\hline & $s=0$ & $s=1$ & $s=2$ \\
\hline$r=2$ & $\xi\left(\Xi^{\langle 10\rangle}-\Xi^{\langle 6,9\rangle}\right)$ & $\xi\left(\Xi^{\langle 5,6\rangle}\right)$ & $\xi\left(\Xi^{\langle 6,7\rangle}\right)$ \\
$r=3$ & $\xi\left(\Xi^{\langle 10\rangle}-\Xi^{\langle 7,9\rangle}\right)$ & $\xi\left(\Xi^{\langle 5,7\rangle}\right)$ & $\xi\left(\Xi^{\langle 6,8\rangle}\right)$ \\
$r=4$ & $\xi\left(\Xi^{\langle 10\rangle}-\Xi^{\langle 8,9\rangle}\right)$ & $\xi\left(\Xi^{\langle 5,8\rangle}\right)$ & $\xi\left(\Xi^{\langle 6,9\rangle}\right)$ \\
$r=5$ & $\xi\left(\Xi^{\langle 10\rangle}-\Xi^{\langle 9\rangle}\right)$ & $\xi\left(\Xi^{\langle 5,9\rangle}\right)$ & $\xi\left(\Xi^{\langle 10\rangle}-\Xi^{\langle 5\rangle}\right)$ \\
\hline & & $s=4$ & $s=5$ \\
\hline$r=2$ & $\xi=3$ & $\xi\left(\Xi^{\langle 8,9\rangle}\right)$ & $\xi\left(\Xi^{\langle 10\rangle}-\Xi^{\langle 5,8\rangle}\right)$ \\
$r=3$ & $\xi\left(\Xi^{\langle 7,8\rangle}\right)$ & $\xi\left(\Xi^{\langle 10\rangle}-\Xi^{\langle 5,7\rangle}\right)$ & $\xi\left(\Xi^{\langle 10\rangle}-\Xi^{\langle 6,8\rangle}\right)$ \\
$r=4$ & $\xi\left(\Xi^{\langle 10\rangle}-\Xi^{\langle 5,6\rangle}\right)$ & $\xi\left(\Xi^{\langle 10\rangle}-\Xi^{\langle 6,7\rangle}\right)$ & $\xi\left(\Xi^{\langle 10\rangle}-\Xi^{\langle 7,8\rangle}\right)$ \\
$r=5$ & $\xi\left(\Xi^{\langle 10\rangle}-\Xi^{\langle 6\rangle}\right)$ & $\xi\left(\Xi^{\langle 10\rangle}-\Xi^{\langle 7\rangle}\right)$ & $\xi\left(\Xi^{\langle 10}-\Xi^{\langle 8\rangle}\right)$ \\
\hline
\end{tabular}

where $\Xi=\Xi_{\gamma} \in \mathbb{M}_{10}(\mathbb{Z})$.

The following formula can be easily obtained from Theorem 2.3.

Corollary 2.5 For any $(\gamma, i, s)$ as above and $l \in \mathbb{N}_{1}$, the dimension vector of the indecomposable object $M_{i, s, l}(\gamma)$ in $\tilde{\mathcal{S}} \subseteq \tilde{\mathcal{H}}$ is given by the expression

$$
\underline{\operatorname{dim}_{k}} M_{i, s, l}(\gamma)=\kappa_{i, s, l}(\gamma)
$$

where

$$
\kappa_{i, s, l}(\gamma):=\mathrm{quo}_{p_{i}}(l) \cdot \xi\left(\Xi_{\gamma}^{\langle 10\rangle}\right)+\kappa_{i, s, \operatorname{rem}_{\mathrm{p}_{\mathrm{i}}}(\mathrm{l})}(\gamma) .
$$


In particular, we have $\operatorname{dim}_{k} M=l \cdot \xi\left(\Xi_{\gamma}^{\langle 10\rangle}\right)$, for any indecomposable object $M$ of quasilength l from homogeneous tubes in the family $\mathcal{T}_{\gamma}$.

(In the formulation above quo $_{p_{i}}(l)$ and $\operatorname{rem}_{p_{i}}(l)$ denote the integer quotient and the remainder of $l$ modulo $p_{i}$, respectively).

Remark 2.6 (a) The dimension vectors of the indecomposables from the tube encoded by the pair $(\gamma, i)=(0,3)$ are known (see [23, (2.3)]. In fact, they can be easily recovered from those for the mouth objects, which are exhibited in [23, (1.3)]. Due to non-stability of the tube the formulas have a slightly irregular shape, so we do not present them here.

(b) There is also an alternative simple description of the dimension vectors of the indecomposables from homogeneous tubes given in terms of the vectors $h_{\gamma}$ (cf. Section 4.4).

\section{7}

As a byproduct of the results above we are able to determine the interval multiplicity of indecomposable objects from $\tilde{\mathcal{S}}$.

More precisely, denote by $\tilde{A}:=\tilde{A}(6)$ the locally bounded category of the bounded quiver $\left(Q^{(\infty)}, \Delta^{(\infty)}(6)\right)$, where $Q^{(\infty)}$ is the infinite linear quiver below

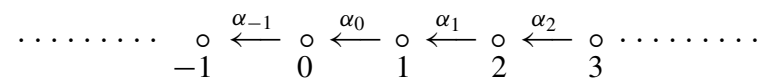

and $\Delta^{(\infty)}(6)$ is the two-sided admissible ideal $\left\langle\alpha_{i+6} \ldots \alpha_{i+1}: i \in \mathbb{Z}\right\rangle$ in the path category $k Q^{(\infty)}$. Recall that the full list $\tilde{\mathbb{S}}=\mathbb{S}_{\tilde{A}}$ of all, up to isomorphism, indecomposable objects in $\bmod (\tilde{A})$ consists of the so-called intervals $I=I_{[i, j]}$, for all $i \leq j$ with $j-i<6$, where the $\tilde{A}$-module $I_{[i, j]}$ is given by setting $I_{l}:=k$, if $i \leq l \leq j, I_{\alpha_{l}}:=\mathrm{id}_{k}$, if $i \leq l<j$, and $I_{l}:=0$ for all $l \in \mathbb{Z} \backslash[i, j]$. Consequently, for any $M$ in $\bmod (\tilde{A})$ we have the interval decomposition $M \cong \bigoplus_{I \in \tilde{\mathbb{S}}} I^{m_{I}}$, where $m_{I}=0$, for almost all $I$ (cf. Section 5.8). The sequence $m(M):=\left(m_{I}\right)_{I \in \tilde{\mathbb{S}}} \in \bigoplus_{\tilde{S}} \mathbb{N}$ depends only on $M$ and it is called the interval multiplicity vector of $M$. Clearly it determines uniquely the isomorphism class of $M$, and conversely.

To formulate our next result observe first that identifying $\tilde{A}$ with the $\mathbb{Z}$-invariant full subcategories $\tilde{A}^{a}$ and $\tilde{A}^{s}$ of $R$, formed by the subsets $\mathbb{Z}$ and $\mathbb{Z}^{\prime}:=\left\{z^{\prime}: z \in \mathbb{Z}\right\}$ of $\tilde{Q}_{0}$, respectively, the modules $M$ in $\bmod (R)$, in particular objects of $\tilde{\mathcal{S}}$, can be presented in the form $M=\left(M^{s}, M^{a}, \varphi_{M}\right)$, where $M^{s}, M^{a}$ belong to $\bmod (\tilde{A})$ and $\varphi_{M}: M^{s} \rightarrow M^{a}$ is an $\tilde{A}$-homomorphism. Due to the formulas of Theorem 2.3 and Corollary 2.5 we are able to determine effectively the interval multiplicity vectors $m(M):=\left(m\left(M^{s}\right), m\left(M^{a}\right)\right)$, for all indecomposable objects $M=\left(M^{s}, M^{a}, \varphi_{M}\right)$ in $\mathcal{D}$ (so in $\tilde{\mathcal{S}}$ ), with the exception of some members of three tubes.

Theorem 2.8 Let $M=\left(M^{s}, M^{a}, \varphi\right)$ be an indecomposable object in $\tilde{\mathcal{S}}$ of the shape $M=$ $M_{i, s, l}(\gamma)$, where $(\gamma, i, s)$ be as in Theorem 2.3 and $l \in \mathbb{N}_{1} ;$ moreover, let $\kappa_{i, s, l}(\gamma):=\left[\begin{array}{l}\left(\hat{d}_{2}\right)_{z \in \mathbb{Z}} \\ \left(\hat{z}_{z}\right)_{z \in \mathbb{Z}}\end{array}\right]$.

(a) If $0<\gamma<1$, then

$$
M^{s} \cong I_{[1,5]} \hat{d}_{5^{\prime}} \oplus I_{[1,4]} \hat{d}_{1^{\prime}}-\hat{d}_{5^{\prime}} \oplus I_{[2,4]} \hat{d}_{4^{\prime}}-\hat{d}_{1^{\prime}} \oplus I_{[2,3]} \hat{d}_{2^{\prime}}-\hat{d}_{4^{\prime}} \oplus I_{[3,3]} \hat{d}_{3^{\prime}}-\hat{d}_{2^{\prime}}
$$


and

$$
\begin{aligned}
M^{a} \cong & I_{[1,6]} \hat{d}_{1} \oplus I_{[2,7]} \hat{d}_{7} \oplus I_{[2,6]} \hat{d}_{6}-\hat{d}_{1}-\hat{d}_{7} \oplus I_{[2,5]} \hat{d}_{2}-\hat{d}_{6} \oplus I_{[3,5]} \hat{d}_{5}-\hat{d}_{2} \\
& \oplus I_{[3,4]^{3}-\hat{d}_{5}} \oplus I_{[4,4]} \hat{d}_{4}-\hat{d}_{3} .
\end{aligned}
$$

(b) If $1<\gamma<\infty$, then

$$
M^{s} \cong I_{[1,5]} \hat{d}_{1^{\prime}} \oplus I_{[2,5]} \hat{d}_{5^{\prime}}-\hat{d}_{1^{\prime}} \oplus I_{[2,4]} \hat{d}_{2^{\prime}}-\hat{d}_{5^{\prime}} \oplus I_{[3,4]} \hat{d}_{4^{\prime}}-\hat{d}_{2^{\prime}} \oplus I_{[3,3]^{\prime}} \hat{d}_{3^{\prime}}-\hat{d}_{4^{\prime}}
$$

and

$$
\begin{aligned}
M^{a} \cong & I_{[1,6]} \hat{d}_{1} \oplus I_{[2,7]} \hat{d}_{7} \oplus I_{[2,6]} \hat{d}_{2}-\hat{d}_{1}-\hat{d}_{7} \oplus I_{[3,6]} \hat{d}_{6}-\hat{d}_{2} \oplus I_{[3,5]} \hat{d}_{3}-\hat{d}_{6} \\
& \oplus I_{[4,5]} \hat{d}_{5}-\hat{d}_{3} \oplus I_{[4,4]} \hat{d}_{4}-\hat{d}_{5} .
\end{aligned}
$$

(c) If $\gamma=1$ and $i=1,2$ then

$$
M^{s} \cong I_{[1,5]} \hat{d}_{5^{\prime}} \oplus I_{[2,4]} \hat{d}_{4^{\prime}}-\hat{d}_{1^{\prime}} \oplus I_{[3,3]} \hat{d}_{3^{\prime}}-\hat{d}_{2^{\prime}}
$$

and

$$
M^{a} \cong I_{[1,6]} \hat{d}_{1} \oplus I_{[2,7]} \hat{d}_{7} \oplus I_{[2,6]} \hat{d}_{6}-\hat{d}_{1}-\hat{d}_{7} \oplus I_{[3,5]} \hat{d}_{5}-\hat{d}_{2} \oplus I_{[4,4]} \hat{d}_{4}-\hat{d}_{3} .
$$

(d) If $\gamma=0$ and $i=1$ then

$$
M^{s} \cong I_{[1,5]} \hat{d}_{5^{\prime}} \oplus I_{[1,4]} \hat{d}_{1^{\prime}}-\hat{d}_{5^{\prime}} \oplus I_{[2,3]} \hat{d}_{2^{\prime}}-\hat{d}_{4^{\prime}}
$$

and

$$
M^{a} \cong I_{[1,6]} \hat{d}_{1} \oplus I_{[2,5]} \hat{d}_{2}-\hat{d}_{6} \oplus I_{[3,4]} \hat{d}_{3}-\hat{d}_{5} .
$$

The proof of the theorem will be given at the end of Section 5. We will also outline there briefly the arguments proving the following.

Corollary 2.9 For any pair $\gamma \in \mathbb{Q}_{0}$ and $l \in \mathbb{N}_{1}$, all the objects $M_{i, s, l p_{i}}(\gamma)$, where $i \in[3]$ and $s \in \mathbb{Z}_{p_{i}}$, provided $\gamma \in \mathbb{Q}_{0} \backslash\{0,1\}, i \in[2]$ and $s \in \mathbb{Z}_{p_{i}}$, provided $\gamma=1$, and respectively, $i=1$ and $s \in \mathbb{Z}_{2}$, provided $\gamma=0$, have the same interval multiplicity vectors. Moreover, these vectors are equal to the vector $m(M)$, for any indecomposable object $M$ of quasi-length l from a homogeneous tube in the family $\mathcal{T}_{\gamma}$.

Remark 2.10 The interval multiplicity vectors for the indecomposables from the exceptional regular tubes, encoded by the pairs $(\gamma, i)=(1,3),(0,2)$, do not have such a homogeneous and nice shape. Nevertheless, they can be reconstructed from those for quasisimple objects in these tubes, with some extra effort (the details will be given in [5]). The quasi-lenght 1 case, however not "completely regular", is easy to handle by the shape of the dimension vectors (see Section 6 , the lists $L^{1}$ and $L^{\infty}$ ). The tube encoded by $(0,3)$ needs a separate treatment, which uses the description of its members given in [23]. 


\section{An Algebra $\Lambda$ and its Realization}

\section{1}

A fundamental role in the understanding of the structure and the description of indecomposable objects of the category $\tilde{\mathcal{S}}=\mathcal{S}(\tilde{6})$ in [23] is played by the algebra $\Lambda:=k Q / \Delta$ of the bounded quiver $(Q, \Delta)$, where $Q$ is the quiver

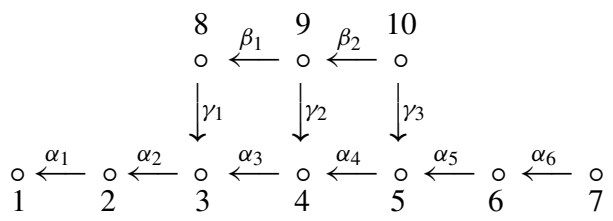

and $\Delta$ is the two-sided admissible ideal $\left\langle\alpha_{6} \ldots \alpha_{1}, \beta_{1} \gamma_{1}-\gamma_{2} \alpha_{3}, \beta_{2} \gamma_{2}-\gamma_{3} \alpha_{4}\right\rangle$ in the path algebra $k Q$ in $Q$ (we follow the definition of $k Q$ from [1, II.1], in [23] $\Lambda$ is denoted by $\Theta$ ). We will always identify the category $\bmod (\Lambda)$ of right finite dimensional $\Lambda$-modules with the category $\operatorname{rep}_{k}(Q, \Delta)$ of finite dimensional representations of $(Q, \Delta)$.

Denote by $\left(Q^{\prime}, \Delta^{\prime}\right)$ the full subquiver of $(\tilde{Q}, \tilde{\Delta})$ formed by the vertices $\left\{1, \ldots, 7,1^{\prime}, \ldots\right.$, $5^{\prime}$ \}. Clearly, $\operatorname{rep}_{k}\left(Q^{\prime}, \Delta^{\prime}\right)$ can be identified (by extension by zeros) with the full subcategory of $\operatorname{rep}_{k}(\tilde{Q}, \tilde{\Delta})$ consisting of all representations $M$ with support contained in $Q^{\prime}$. To any $M=$ $\left(M_{v}, M_{\alpha}\right)_{v \in Q_{0}, \alpha \in Q_{1}}$ in $\bmod (\Lambda)$ we associate the representation $\hat{M}=\left(\hat{M}_{w}, \hat{M}_{\beta}\right)_{w \in Q_{0}^{\prime}, \beta \in Q_{1}^{\prime}}$ in $\operatorname{rep}_{k}\left(Q^{\prime}, \Delta^{\prime}\right)$ defined as follows: $\hat{M}_{v}:=M_{v}$ and $\hat{M}_{\alpha}:=M_{\alpha}$ whenever this makes sense, also under the additional identifications $3^{\prime}=8,4^{\prime}=9,5^{\prime}=10$ of vertices and $\alpha_{3}^{\prime}=\beta_{1}$, $\alpha_{4}^{\prime}=\beta_{2}, v_{3}=\gamma_{1}, v_{4}=\gamma_{2}, v_{5}=\gamma_{3}$ of arrows; moreover, we set $\hat{M}_{1^{\prime}}:=M_{1}, \hat{M}_{2^{\prime}}:=M_{2}$, $\hat{M}_{\nu_{1}}:=\operatorname{id}_{M_{1}}, \hat{M}_{\nu_{2}}:=\operatorname{id}_{M_{2}}, \hat{M}_{\alpha_{2}^{\prime}}:=M_{\alpha_{2}} \circ M_{\gamma_{1}}$ and $\hat{M}_{\alpha_{1}^{\prime}}:=M_{\alpha_{1}} \circ M_{\alpha_{2}} \circ M_{\gamma_{1}}$.

The following fact $[23,1]$ shows the role of $\Lambda$ in studying of the category $\tilde{\mathcal{S}}$.

Lemma 3.2 The functorial mapping $M \mapsto \hat{M}$ yields equivalences $\bmod (\Lambda) \simeq \mathcal{A}$ and $\operatorname{rep}_{k}^{\bullet}(Q, \Delta) \simeq \mathcal{A}^{\bullet}$ of categories, where $\mathcal{A}$ denotes the full subcategory of $\operatorname{rep}_{k}\left(Q^{\prime}, \Delta^{\prime}\right)$ formed by all $M^{\prime}$ such that $M_{\nu_{1}}^{\prime}$ and $M_{\nu_{2}}^{\prime}$ are isomorphisms, $\operatorname{rep}_{k}^{\bullet}(Q, \Delta)$ the full subcategory of $\bmod (\Lambda)$ formed by all $M$ such that $M_{\gamma_{1}}, M_{\gamma_{2}}, M_{\gamma_{3}}$ are monomorphisms, and $\mathcal{A}^{\bullet}:=$ $\mathcal{A} \cap \tilde{\mathcal{S}}$. Both subcategories, $\mathcal{A}$ and $\mathcal{A}^{\bullet}$, are closed under extensions in $\tilde{\mathcal{H}}=\bmod (R)$.

\section{3}

Recall that $\Lambda$ is a tubular algebra, in the sense of [19], of type $(2,3,6)$ (see [23]). The structure of the Auslander-Reiten quiver $\Gamma_{\Lambda}$ of $\bmod (\Lambda)$ looks as follows:

$$
\Gamma_{\Lambda}=\mathcal{P} \sqcup\left(\coprod_{\gamma \in \overline{\mathbb{Q}}_{0}} \mathcal{T}_{(\gamma)}\right) \sqcup \mathcal{Q}
$$

where

- $\mathcal{P}$ is a preprojective component which coincides with the preprojective component of $\bmod \left(\Lambda_{0}\right)$, 
- $\quad \mathcal{T}=\left(\mathcal{T}_{(\gamma)}\right)_{\gamma \in \overline{\mathbb{Q}}_{0}}$ consists of separating $\mathbb{P}^{1}(k)$-families of tubes of type $(2,3,6)$, all but $\mathcal{T}_{(0)}$ and $\mathcal{T}_{(\infty)}$ consisting only of stable ones (i.e. not containing a projective or injective module),

- $\mathcal{Q}$ is a preinjective component which coincides with the preinjective component of $\bmod \left(\Lambda_{\infty}\right)$.

(Here $\overline{\mathbb{Q}}_{0}:=\{q \in \overline{\mathbb{Q}}: q \geq 0\} ; \Lambda_{0}$ and $\Lambda_{\infty}$ denote the tame concealed algebras of the bounded quivers obtained from $(Q, \Delta)$ by deleting the vertex sets $\{7,10\}$ and $\{1\}$, respectively).

The tubular families in $\Gamma_{\Lambda}$ play a basic role in the description of $\mathcal{D}$. More precisely, for every $\gamma \in \mathbb{Q}_{+}:=\mathbb{Q}_{0} \backslash\{0\}$, the $\mathbb{P}^{1}(k)$-family $\mathcal{T}_{\gamma}$ in $\tilde{\mathcal{S}}$ is just equal to the image $\hat{\mathcal{T}}_{(\gamma)}$ of the family $\mathcal{T}_{(\gamma)}$ via the functor $(\hat{-})$. Similarly, the same holds for $\mathcal{T}_{0}^{\prime}$ and $\mathcal{T}_{(0)}^{\prime}$, which consist of all regular tubes in $\mathcal{T}_{0}$ and $\mathcal{T}_{(0)}$, respectively. (In both cases we delete the unique non-regular tube, i.e. this of rank 6). Notice also that the rank-6 tubes in $\mathcal{T}_{0}$ and $\mathcal{T}_{(0)}$ (and in $\mathcal{T}_{(\infty)}$ ) are also strongly related (see $[23,1]$ for the details).

\section{4}

In the proof of our main result we need not only the fact that $\Lambda$ is a tubular algebra of type $(2,3,6)$, but also a realization of $\Lambda$ as the endomorphism algebra of a concrete tilting bundle $T=\bigoplus_{i=1}^{10} T_{i}$ in $\operatorname{coh}(\mathbb{X})$, where $\mathbb{X}$ is a weighted projective line of tubular weighed type $(2,3,6)$ (see Theorem 3.6, cf. [15, Proposition 3.6]). This allows us to treat $\Lambda$-modules (in consequence some objects of $\tilde{\mathcal{S}})$ as objects of $\mathcal{D}^{b} \operatorname{coh}(\mathbb{X})$, and to apply techniques developed for studying coherent sheaves to our problem.

In the construction of this realization we use rather detailed information on the structure of the category of graded sheaves over weighted projectives lines, introduced by Geigle and Lenzing [7] for a better understanding of the module theory over canonical algebras of Ringel [19]. Their idea is based on associating to a canonical algebra $C=C(\mathbf{p}, \lambda)$ defined by the sequences $\mathbf{p}=\left(p_{1}, \ldots, p_{t}\right) \in \mathbb{N}^{t}$ and $\lambda=\left(\lambda_{1}, \ldots, \lambda_{t}\right) \in\left(\mathbb{P}^{1}(k)\right)^{t}$ consisting of pairwise different points $\lambda_{i}$, the so-called weighted projective line $\mathbb{X}=\mathbb{X}(\mathbf{p}, \lambda)$. $\mathbb{X}$ is defined as the graded projective spectrum $\mathbb{X}:=\operatorname{Proj}^{\mathbb{L}}(S)$, where $S$ is the commutative $k$-algebra

$$
S=S(\mathbf{p}, \lambda):=k\left[X_{1}, \ldots, X_{t}\right] /\left(\left\{X_{i}^{p_{i}}-X_{1}^{p_{1}}-\lambda_{i} X_{2}^{p_{2}}\right\}_{i=3, \ldots, t}\right)
$$

admitting a natural grading $S=\bigoplus_{\vec{x} \in \mathbb{L}} S_{\vec{x}}$ by the rank-one abelian ordered group $\mathbb{L}=\mathbb{L}(\mathbf{p})$ on generators $\vec{x}_{1}, \vec{x}_{2}, \ldots, \vec{x}_{t}$ with relations $p_{1} \vec{x}_{1}=p_{2} \vec{x}_{2}=\cdots=p_{t} \vec{x}_{t}=: \vec{c}$, such that the degree of each $X_{i}$ is just $\vec{x}_{i}$. Roughly speaking $\mathbb{X}(\mathbf{p}, \lambda)$ consists of exceptional points $x_{1}, \ldots, x_{t}$, corresponding to $\lambda_{1}, \ldots, \lambda_{t}$, of multiplicities $p\left(x_{1}\right)=p_{1}, \ldots, p\left(x_{t}\right)=p_{t}$, and the remaining points corresponding to $x=x_{1}^{p_{1}}+\lambda x_{2}^{p_{2}}$, for $\lambda \in \mathbb{P}^{1}(k) \backslash\left\{\lambda_{1}, \ldots, \lambda_{t}\right\}$, which are ordinary and have multiplicity $p(x)=1$. (One can identify points $x \in \mathbb{X}$ with the corresponding $\lambda \in \mathbb{P}^{1}(k)$, as above). Then one considers the abelian hereditary category $\operatorname{coh}(\mathbb{X})$ consisting of all $\mathbb{L}$-graded coherent sheaves over $\mathbb{X}$. The sheaf $T_{\mathcal{O}}:=\bigoplus_{0 \leq \vec{x} \leq \vec{c}} \mathcal{O}(\vec{x})$, formed by summing up all shifted structure sheaves $\mathcal{O}(\vec{x}):=\mathcal{O}_{\mathbb{X}}(\vec{x})$ such that $0 \leq \vec{x} \leq \vec{c}$, is a tilting object in $\operatorname{coh}(\mathbb{X})$ with $\operatorname{End}_{\mathbb{X}}\left(T_{\mathcal{O}}\right) \cong C$, where the partial order $\leq$ on $\mathbb{L}$ is determined by $\mathbb{L}_{+}:=\sum_{i=1}^{t} \mathbb{N} \vec{x}_{i}$. Consequently, $T_{\mathcal{O}}$ allows to define in a standard way a triangle equivalence $\mathcal{D}^{b} \operatorname{coh}(\mathbb{X}) \simeq \mathcal{D}^{b} \bmod (C)$ (see [7] for all details).

The category $\operatorname{coh}(\mathbb{X})$ admits Serre duality [7]. As a consequence coh $(\mathbb{X})$ has almost split sequences, the Auslander-Reiten translation $\tau_{\mathbb{X}}$ in $\operatorname{coh}(\mathbb{X})$ is given by shift with the dualizing element $\vec{\omega}=(t-2) \vec{c}-\sum_{i=1}^{t} \vec{x}_{i}$. Similarly as in the case of a smooth projective curve, for coherent sheaves $\mathcal{F}$ over $\mathbb{X}$ we have concepts of rank and degree, defined as integers 
$\operatorname{rk}(\mathcal{F}) \in \mathbb{N}$ and $\operatorname{deg}(\mathcal{F}) \in \mathbb{Z}$ (see [7, 1.8 and 2.8]). The rank and degree functions induce $\mathbb{Z}$ linear forms rk, deg : $\mathrm{K}_{0}(\mathbb{X}) \rightarrow \mathbb{Z}$, where $\mathrm{K}_{0}(\mathbb{X})$ is the Grothendieck group of the category $\operatorname{coh}(\mathbb{X})$, which is a free abelian group with a standard natural $\mathbb{Z}$-basis $\mathcal{B}_{\mathcal{O}}:=\{[\mathcal{O}(\vec{x})]$ : $\overrightarrow{0} \leq \vec{x} \leq \vec{c}$ \} (see Section 3.5 for their matrices in case $(2,3,6)$ ). Moreover, it is equipped with the $\mathbb{Z}$-bilinear form eulf : $\mathrm{K}_{0}(\mathbb{X})^{2} \rightarrow \mathbb{Z}$, called the Euler form, given by the formula $\operatorname{eulf}([\mathcal{F}],[\mathcal{G}])=\operatorname{dim}_{k} \operatorname{Hom}_{\mathbb{X}}(\mathcal{F}, \mathcal{G})-\operatorname{dim}_{k} \operatorname{Ext}_{\mathbb{X}}^{1}(\mathcal{F}, \mathcal{G})$, for $\mathcal{F}, \mathcal{G}$ in $\operatorname{coh}(\mathbb{X})$. For a nonzero indecomposable coherent sheaf $\mathcal{F}$ we define the slope $\mu(\mathcal{F})=\frac{\operatorname{deg}(\mathcal{F})}{\operatorname{rk}(\mathcal{F})}$ as an element of $\overline{\mathbb{Q}}$.

Recall from [7] that each indecomposable element in $\operatorname{coh}(\mathbb{X})$ is a locally free sheaf, called a vector bundle, or a sheaf of finite length. Denote by vect $(\mathbb{X})\left(\right.$ respectively $\left.\operatorname{coh}_{0}(\mathbb{X})\right)$ the category of vector bundles (respectively finite length sheaves) on $\mathbb{X}$ and, for any $q \in \overline{\mathbb{Q}}=$ $\mathbb{Q} \cup\{\infty\}$, by $\mathcal{C}_{q}$ the full subcategory of $\operatorname{coh}(\mathbb{X})$ formed by all sheaves whose indecomposable summands $\mathcal{F}$ satisfy the equality $\mu(\mathcal{F})=q$. Then $\operatorname{coh}(\mathbb{X})=\operatorname{vect}(\mathbb{X}) \vee \operatorname{coh}_{0}(\mathbb{X}), \operatorname{coh}_{0}(\mathbb{X})=$ $\mathcal{C}_{\infty}$ and $\operatorname{vect}(\mathbb{X})=\bigvee_{q \in \mathbb{Q}} \mathcal{C}_{q}$. It is also known that

$$
\operatorname{coh}_{0}(\mathbb{X}) \cong \coprod_{x \in \mathbb{X}} \bmod _{0}^{\mathbb{L}} \mathcal{O}_{\mathbb{X}, x}
$$

and that for each $x \in \mathbb{X}$ the category of $\mathbb{L}$-graded finite length modules $\bmod _{0}^{\mathbb{L}} \mathcal{O}_{\mathbb{X}, x}$ over the stalk $\mathcal{O}_{\mathbb{X}, x}$ is a uniserial category having $p(x)$ simples. Moreover, all $\mathcal{C}_{q}$, for $q \in \overline{\mathbb{Q}}$, are abelian categories, additionally uniserial (with a length function $\ell=\ell_{q}$ called quasilength), enjoying the same tubular structure of the Auslander-Reiten quiver as $\operatorname{coh}_{0}(\mathbb{X})$, if $\mathbf{p}$ is of tubular type (e.g. for $\mathbf{p}=(2,3,6)$ ). Recall also that then, due to semistability arguments, for any indecomposable $\mathcal{F}, \mathcal{G}$ in $\operatorname{coh}(\mathbb{X})$ we have always

$$
\mu(\mathcal{F})>\mu(\mathcal{G}) \Rightarrow \operatorname{Hom}_{\mathbb{X}}(\mathcal{F}, \mathcal{G})=0 .
$$

Moreover, we have the formulas

$$
\operatorname{rk}(\mathcal{F})=r \frac{p_{t}}{\rho(\mathcal{F})} \ell(\mathcal{F}), \quad \operatorname{deg}(\mathcal{F})=d \frac{p_{t}}{\rho(\mathcal{F})} \ell(\mathcal{F})
$$

where $\mu(\mathcal{F})=\frac{d}{r}$ is an irreducible fraction presentation of the slope of $\mathcal{F}$ and $\rho(\mathcal{F})$ denotes

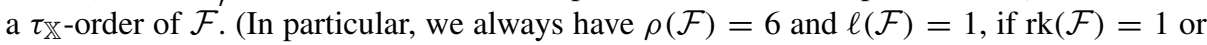
$\operatorname{deg}(\mathcal{F})=1$, for $\mathbf{p}=(2,3,6)$, see e.g. [14]).

\section{5}

From now on, if not restated, we assume that $\mathbf{p}=(2,3,6)$. Then the Auslander-Reiten quiver of each category $\mathcal{C}_{q}$ consists of $\mathbb{P}^{1}(k)$-families $\mathcal{T}^{q}(\mathbb{X})=\left\{\mathcal{T}_{\lambda}^{q}(\mathbb{X})\right\}_{\lambda \in \mathbb{X}}$ of pairwise orthogonal standard stable tubes, which are almost all homogeneous except of three of them $\mathcal{T}_{\lambda_{1}}^{q}(\mathbb{X}), \mathcal{T}_{\lambda_{2}}^{q}(\mathbb{X}), \mathcal{T}_{\lambda_{3}}^{q}(\mathbb{X})$, being of the ranks 2,3 and 6 , respectively. We will consider $\mathcal{B}_{\mathcal{O}}$ as the ordered basis of the shape

$$
\mathcal{B}_{\mathcal{O}}=\left([\mathcal{O}],\left[\mathcal{O}\left(\vec{x}_{1}\right)\right],\left[\mathcal{O}\left(\vec{x}_{2}\right)\right],\left[\mathcal{O}\left(2 \vec{x}_{2}\right)\right],\left[\mathcal{O}\left(\vec{x}_{3}\right)\right], \ldots,\left[\mathcal{O}\left(5 \vec{x}_{3}\right)\right],[\mathcal{O}(\vec{c})]\right) .
$$

Moreover, the matrices $\underline{\mathrm{rk}}_{\mathcal{B}_{\mathcal{O}}}$ and $\underline{\operatorname{deg}}_{\mathcal{B}_{\mathcal{O}}}$ of the rank and degree homomorphisms with respect to the basis $\mathcal{B}_{\mathcal{O}}$ (and the standard basis $\{1\}$ of $\mathbb{Z}$ ) are given as follows:

$$
\underline{\mathrm{rk}}_{\mathcal{B}_{\mathcal{O}}}=[1, \ldots, 1], \quad \underline{\operatorname{deg}}_{\mathcal{B}_{\mathcal{O}}}=[0,3,2,4,1,2,3,4,5,6] .
$$

Finally, the matrix $G_{\mathcal{B}_{\mathcal{O}}} \in \mathbb{M}_{10}(\mathbb{Z})$ of the Euler form on $\mathrm{K}_{0}(\mathbb{X})$ with respect to $\mathcal{B}_{\mathcal{O}}$ coincides with the Cartan matrix of the canonical algebra of type $(2,3,6)$, where the ordering of the projective indecomposable modules corresponds to that in the sequence $\mathcal{B}_{\mathcal{O}}$. 
For any $i=1,2,3$ and $j \in \mathbb{Z}_{p_{i}}$ we set

$$
\mathcal{S}_{i, j}:=\operatorname{Coker}\left(\mathcal{O}\left(j \vec{x}_{i}\right) \stackrel{x_{i}}{\longrightarrow} \mathcal{O}\left((j+1) \vec{x}_{i}\right)\right) .
$$

All $\mathcal{S}_{i, j}$ 's are simple torsion sheaves. For each $i=1,2,3$, the set $\left\{\mathcal{S}_{i, j}\right\}_{j \in \mathbb{Z}_{p_{i}}}$ forms a full list of consecutive with respect to $\tau_{\mathbb{X}}^{-1}$ quasi-simple objects in the exceptional tubes in $\mathcal{C}_{\infty}$.

The families $\left\{\mathcal{S}_{i, j}\right\}$ yield another ordered basis

$$
\mathcal{B}_{\mathcal{S}}:=\left([\mathcal{O}],\left[\mathcal{S}_{1,1}\right],\left[\mathcal{S}_{2,1}\right],\left[\mathcal{S}_{2,2}\right],\left[\mathcal{S}_{3,1}\right], \ldots,\left[\mathcal{S}_{3,5}\right],[\mathcal{S}]\right)
$$

of $\mathrm{K}_{0}(\mathbb{X})$, which is suitable from the computational point of view, where $[\mathcal{S}]:=$ $\sum_{j \in \mathbb{Z}_{p_{i}}}\left[\mathcal{S}_{i, j}\right]$ (note that the definition does not depend on the choice of $i$ ).

Theorem 3.6 The algebra $\Lambda$ admits a realization $\Lambda \cong \operatorname{End}_{\mathbb{X}}(T)$, where $T=\bigoplus_{i=1}^{10} T_{i}$ is the unique, up to isomorphism, tilting sheaf over a weighted projective line $\mathbb{X}$ of the tubular type $(2,3,6)$, having the following properties:

(a) the sequences $\underline{\operatorname{rk}}(T)=\left(\operatorname{rk}\left(T_{i}\right)\right)_{i \in[10]}, \operatorname{deg}(T)=\left(\operatorname{deg}\left(T_{i}\right)\right)_{i \in[10]}$ and $\mu(T)=$ $\left(\mu\left(T_{i}\right)\right)_{i \in[10]}$ of ranks, degrees and slopes of indecomposable direct summands $T_{i}$ of $T, i \in[10]$, have the form $\underline{\operatorname{rk}}(T)=(1,3,5,6,5,3,1,4,3,1), \underline{\operatorname{deg}}(T)=$ $(0,1,2,3,3,2,1,2,2,1)$ and $\underline{\mu}(T)=\left(0, \frac{1}{3}, \frac{2}{5}, \frac{1}{2}, \frac{3}{5}, \frac{2}{3}, 1, \frac{1}{2}, \frac{2}{3}, 1\right)$, respectively.

(b) the coordinate vectors $\left[T_{i}\right]_{\mathcal{B}_{\mathcal{S}}}$ of the Grothendieck classes $\left[T_{i}\right] \in \mathrm{K}_{0}(\mathbb{X})$ of $T_{i}$ with respect of the basis $\mathcal{B}_{\mathcal{S}}$, for $i \in[10]$, are equal to the consecutive columns of the matrix

$$
\Theta=\left[\begin{array}{rrrrrrrrrr}
1 & 3 & 5 & 6 & 5 & 3 & 1 & 4 & 3 & 1 \\
-1 & -2 & -3 & -4 & -3 & -2 & -1 & -2 & -2 & -1 \\
0 & -1 & -2 & -2 & -2 & -1 & -1 & -1 & -1 & 0 \\
-1 & -2 & -4 & -4 & -4 & -2 & -1 & -3 & -2 & -1 \\
0 & 0 & -1 & -1 & -1 & -1 & 0 & 0 & 0 & 0 \\
0 & -1 & -2 & -2 & -2 & -2 & -1 & -1 & 0 & 0 \\
0 & -1 & -3 & -3 & -2 & -2 & -1 & -2 & -1 & 0 \\
0 & -1 & -3 & -4 & -3 & -2 & -1 & -2 & -1 & 0 \\
-1 & -2 & -4 & -5 & -4 & -3 & -1 & -3 & -2 & 0 \\
1 & 3 & 6 & 7 & 6 & 4 & 2 & 4 & 3 & 1
\end{array}\right] .
$$

Remark 3.7 The shape of the sequences $\underline{\operatorname{rk}}(T)$ and $\operatorname{deg}(T)$ (consequently of $\underline{\mu}(T)$ ), for the tilting sheaf $T$ realizing $\Lambda$ as $E:=\operatorname{End}_{\mathbb{X}}(T)$, under some natural "normalizing conditions" for $T$ is canonically determined. One can show that if $T$ is a tilting bundle, then the equality $\left[\operatorname{rk}\left(T_{l}\right)\right]_{l \in[10]}=h_{1}(:=[1,3,5,6,5,3,1 ; 4,3,1])$ always holds, once we assume that $\operatorname{coh}_{0}(\mathbb{X})=<\mathcal{T}^{\infty}(\mathbb{X})>$ corresponds via the triangle equivalence $\Psi: \mathcal{D}^{b} \operatorname{coh}(\mathbb{X}) \stackrel{\simeq}{\longrightarrow}$ $\mathcal{D}^{b} \bmod (E)$ induced by $T$, to the full subcategory $<\mathcal{T}_{(1)}>$ of $\bmod (\Lambda)$ formed by all objects from the central family $\mathcal{T}_{(1)}$ (see Sections 4.4 and 4.1 for definitions of $h_{1}$ and $\Psi$, respectively). Moreover, if additionally $\mu\left(T_{1}\right)=0$ then we have $\left[\operatorname{deg}\left(T_{l}\right)\right]_{l \in[10]}=h_{\infty}$. In fact, these two equalities follow from some formula which holds for tilting sheaves over all weighted projective lines of tubular type, and can be treated as a tool for finding $\underline{\operatorname{rk}}(T)$ and $\operatorname{deg}(T)$ of potential "tilted realization" of a given tubular algebra in general situations (this

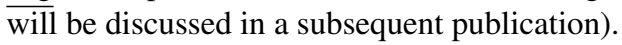

The proof of Theorem 3.6, we present below (see Section 3.12), has a $K$-theoretical character and uses the technique of the so-called telescoping functors [14]. We start by a 
rather general fact which says how to determine, in some nice situations, the Cartan matrix of the algebra $\operatorname{End}_{\mathbb{X}}\left(\bigoplus_{i \in[10]} T_{i}\right)$ via the matrix of the respective values of the Euler form.

Lemma 3.8 Let $\tilde{T}:=\left\{T_{i}\right\}_{i=1, \ldots, n}$ be a family of pairwise nonisomorphic quasi-simple sheaves in $\operatorname{coh}(\mathbb{X})$ lying in exceptional tubes, where $\mathbb{X}$ is a weighted projective line of weight type $\boldsymbol{p}=\left(p_{1}, \ldots, p_{t}\right)$ and $n=2+\sum_{l=1}^{t}\left(p_{l}-1\right)$. Assume that $\tilde{T}$ satisfies the following two conditions:

(a) if $\mu\left(T_{i}\right)<\mu\left(T_{j}\right)$ then eulf $\left(\left[T_{j}\right],\left[T_{i}\right]\right) \geq 0$,

(b) if $\mu\left(T_{i}\right)=\mu\left(T_{j}\right)$, for $i \neq j$, then $T_{i} \neq \tau_{\mathbb{X}}\left(T_{j}\right), \tau_{\mathbb{X}}^{-1}\left(T_{j}\right)$.

Then $T:=\bigoplus_{i=1}^{n} T_{i}$ is a tilting sheaf. In particular, the Cartan matrix $C_{E}=\left[c_{i, j}^{E}\right] \in$ $\mathbb{M}_{n}(\mathbb{Z})$ of the endomorphism algebra $E:=\operatorname{End}_{\mathbb{X}}(T)$ is given by the formula $c_{i, j}^{E}=$ eulf([ $\left.\left.T_{i}\right],\left[T_{j}\right]\right)$, for all $i, j=1, \ldots, n$.

Proof If $\mu\left(T_{i}\right)<\mu\left(T_{j}\right)$ then due to formula 3.4(*) we have $\operatorname{Hom}_{\mathbb{X}}\left(T_{j}, T_{i}\right)=0$ and by Serre duality also $\operatorname{Ext}_{\mathbb{X}}^{1}\left(T_{i}, T_{j}\right) \cong D\left(\operatorname{Hom}_{\mathbb{X}}\left(T_{j}, \tau_{\mathbb{X}} T_{i}\right)\right)=0$. Hence, due to (a), it follows that $0 \leq \operatorname{eulf}\left(\left[T_{j}\right],\left[T_{i}\right]\right)=-\operatorname{dim}_{k} \operatorname{Ext}_{\mathbb{X}}^{1}\left(T_{j}, T_{i}\right)$, so $\operatorname{Ext}_{\mathbb{X}}^{1}\left(T_{j}, T_{i}\right)=0$. Consequently, $\operatorname{Ext}_{\mathbb{X}}^{1}\left(T_{i}, T_{j}\right)=0$, provided $\mu\left(T_{i}\right) \neq \mu\left(T_{j}\right)$. Similarly, if $\mu\left(T_{i}\right)=\mu\left(T_{j}\right)$, for $i \neq j$, then due to (b) and Serre duality we have $\operatorname{Ext}_{\mathbb{X}}^{1}\left(T_{i}, T_{j}\right)=0=\operatorname{Ext}_{\mathbb{X}}^{1}\left(T_{j}, T_{i}\right)$, since $T_{i}, T_{j}$ are quasi-simple. Finally, $\operatorname{Ext}_{\mathbb{X}}^{1}\left(T_{i}, T_{i}\right)=0$ for every $i$, since each $T_{i}$ is exceptional as a quasisimple object in an exceptional tube. Consequently, $T$ is a partial tilting sheaf and $c_{i, j}^{E}=$ eulf([ $\left.\left.T_{i}\right],\left[T_{j}\right]\right)$, for all $i, j$, since $c_{i, j}^{E}=\operatorname{dim}_{k} \operatorname{Hom}_{E}\left(\operatorname{Hom}_{\mathbb{X}}\left(T, T_{i}\right), \operatorname{Hom}_{\mathbb{X}}\left(T, T_{j}\right)\right)=$ $\operatorname{dim}_{k} \operatorname{Hom}_{\mathbb{X}}\left(T_{i}, T_{j}\right)$. The Grothendieck group $\mathrm{K}_{0}(\mathbb{X})$ of the category $\operatorname{coh}(\mathbb{X})$ is a free abelian group of rank $n$ so $T$ is a tilting sheaf due to the analogon of Bongartz's lemma for $\operatorname{coh}(\mathbb{X})$ (see e.g. [1, Lemma VI.2.4], or [15, Lemma 2.1]).

Our next result deals with the problem of a realization of $\Lambda$ as an endomorphism algebra of a tilting bundle. Before we formulate it observe that the Cartan matrix $C_{\Lambda}:=\left[c_{i, j}^{\Lambda}\right] \in$ $\mathbb{M}_{10}(\mathbb{Z})$ of $\Lambda$ has the form

$$
C_{\Lambda}=\left[\begin{array}{llllllllll}
1 & 1 & 1 & 1 & 1 & 1 & 0 & 1 & 1 & 1 \\
0 & 1 & 1 & 1 & 1 & 1 & 1 & 1 & 1 & 1 \\
0 & 0 & 1 & 1 & 1 & 1 & 1 & 1 & 1 & 1 \\
0 & 0 & 0 & 1 & 1 & 1 & 1 & 0 & 1 & 1 \\
0 & 0 & 0 & 0 & 1 & 1 & 1 & 0 & 0 & 1 \\
0 & 0 & 0 & 0 & 0 & 1 & 1 & 0 & 0 & 0 \\
0 & 0 & 0 & 0 & 0 & 0 & 1 & 0 & 0 & 0 \\
0 & 0 & 0 & 0 & 0 & 0 & 0 & 1 & 1 & 1 \\
0 & 0 & 0 & 0 & 0 & 0 & 0 & 0 & 1 & 1 \\
0 & 0 & 0 & 0 & 0 & 0 & 0 & 0 & 0 & 1
\end{array}\right] .
$$

Proposition 3.9 Let $T=\bigoplus_{i=1}^{n} T_{i}$ be a tilting sheaf in $\operatorname{coh}(\mathbb{X})$ with endomorphism algebra $E:=\operatorname{End}_{\mathbb{X}}(T)$, where $\mathbb{X}$ is a weighted projective line of tubular weight type $\boldsymbol{p}=(2,3,6)$. Assume that $T$ satisfies the following two conditions:

- $\underline{\operatorname{rk}}(T)=(1,3,5,6,5,3,1,4,3,1)$, where $\underline{\operatorname{rk}}(T):=\left(\operatorname{rk}\left(T_{1}\right), \ldots, \operatorname{rk}\left(T_{10}\right)\right)$,

- $C_{E}=C_{\Lambda}$; more precisely, $\operatorname{dim}_{k} \operatorname{Hom}_{\mathbb{X}}\left(T_{i}, T_{j}\right)=c_{i, j}^{\Lambda}$ for all $i, j$. 
Then we have $E \cong \Lambda$, provided additionally $\operatorname{deg}\left(T_{2}\right)=\operatorname{deg}\left(T_{10}\right)$.

Proof To prove the assertion it suffices to construct a surjective algebra homomorphism $\psi: k Q \rightarrow E$ such that $\psi(\Delta)=0$. The equality $C_{\Lambda}=C_{E}$ implies in particular that $\operatorname{dim}_{k} \Lambda=\operatorname{dim}_{k} E$; hence, the homomorphism $\bar{\psi}: k Q / \Delta \rightarrow E$ induced by $\psi$ is then an isomorphism.

Denote by $\Omega$ the set consisting of all 35 pairs $(i, j) \in[10]^{2}$ such that $i \neq j$ and $c_{i, j}^{E}=$ $c_{i, j}^{\Lambda} \neq 0$ (hence equal to 1 ). For any $(i, j) \in \Omega$ we fix a nonzero map $\theta_{j, i} \in \operatorname{Hom}_{\mathbb{X}}\left(T_{i}, T_{j}\right)$ (clearly, $\operatorname{Hom}_{\mathbb{X}}\left(T_{i}, T_{j}\right)=k \theta_{j, i}$ ). From the shape of $C_{E}$ it follows that we have the following configuration of morphisms in $\operatorname{coh}(\mathbb{X})$

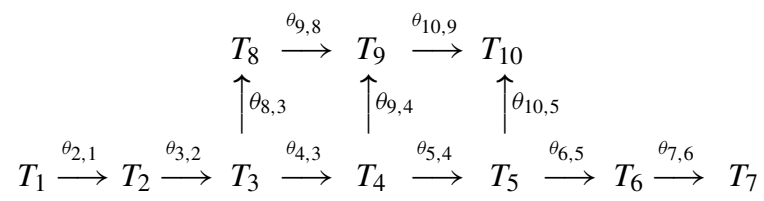

which are irreducible in the full subcategory of $\operatorname{coh}(\mathbb{X})$ formed by $\tilde{T}=\left\{T_{i}\right\}_{i=1, \ldots, n}$. It occurs that the remaining maps $\theta_{j, i}$ are equal, up to nonzero scalars, to compositions of these from the diagram above.

Let $\Omega^{\prime}$ be the following 11 element set:

$$
\Omega^{\prime}:=\{(1,2),(2,3),(3,4),(4,5),(5,6),(6,7),(3,8),(4,9),(5,10),(8,9),(9,10)\} .
$$

We show that for each pair $(i, j) \in \Omega$ and each sequence $i_{0}=i, i_{1}, \ldots, i_{m}=j$, with $m \geq 1$, such that $\left(i_{l-1}, i_{l}\right) \in \Omega^{\prime}$ for all $l=1, \ldots, m$, the composed map $\tilde{\theta}_{\left(i_{m}, \ldots, i_{0}\right)}:=$ $\theta_{i_{m}, i_{m-1}} \circ \ldots \circ \theta_{i_{1}, i_{0}}$ is nonzero. Note that

(i): for each pair $(i, j) \in \Omega$ such a sequence always exists.

We start by formulating an analogon of [9, Lemma 4.1] for $\operatorname{coh}(\mathbb{X})$, which in the sheaf case can be made more precise. Namely, if $T=\bigoplus_{i=1}^{n} T_{i}$ is a tilting sheaf with $T_{i}$ indecomposable then each nonzero morphism $\theta: T_{i} \rightarrow T_{j}$, for $i \neq j$, is either a monomorphism or an epimorphism. Moreover, in case $T$ is a bundle, due to the properties of the rank function we have the following:

(ii): $0 \neq \theta \in \operatorname{Hom}_{\mathbb{X}}\left(T_{i}, T_{j}\right)$, for $i \neq j$, is a monomorphism (respectively, an epimorphism) if and only if $\operatorname{rk}\left(T_{i}\right) \leq \operatorname{rk}\left(T_{j}\right)$ (respectively, $\left.\operatorname{rk}\left(T_{i}\right)>\operatorname{rk}\left(T_{j}\right)\right)$.

Next we notice that for any nonzero monomorphism $f: A \rightarrow B$ and any epimorphism $g: B \rightarrow C$ in $\operatorname{coh}(\mathbb{X})$ the following implication holds true

(iii) :

$$
\operatorname{rk}(A)+\operatorname{rk}(C)>\operatorname{rk}(B) \Longrightarrow g \circ f \neq 0 .
$$

Indeed, if $g \circ f=0$ then the sequence $0 \rightarrow A \stackrel{f}{\rightarrow} B \stackrel{\pi}{\rightarrow}$ Coker $f \rightarrow 0$ is exact and $g$ admits a factorization $g=\pi g^{\prime}$ through the epimorphism $g^{\prime}$ : Coker $f \rightarrow C$; hence $\operatorname{rk}(B)=\operatorname{rk}(A)+\operatorname{rk}($ Coker $f) \geq \operatorname{rk}(A)+\operatorname{rk}(C)$, a contradiction.

Now, applying these two general remarks we show the major part of our claim. First we observe that the maps $\tilde{\theta}_{(3, \ldots, 1)}, \tilde{\theta}_{(4, \ldots, 2)}, \tilde{\theta}_{(4, \ldots, 1)}$ are nonzero monomorphisms as compositions of the nonzero maps $\theta_{2,1}, \theta_{3,2}, \theta_{4,3}$, which by (ii) are monomorphisms; respectively, $\tilde{\theta}_{(6, \ldots, 4)}, \tilde{\theta}_{(7, \ldots, 5)}, \tilde{\theta}_{(7, \ldots, 4)}, \tilde{\theta}_{(10, \ldots, 8)}, \tilde{\theta}_{(9,8,3)}, \tilde{\theta}_{(10,9,4)}, \tilde{\theta}_{(10,5,4)}$ and $\tilde{\theta}_{(10,9,8,3)}$ are nonzero epimorphisms as composition of nonzero maps $\theta_{5,4}, \theta_{6,5}, \theta_{7,6}, \theta_{8,3}, \theta_{9,4}, \theta_{10,5}, \theta_{9,8}, \theta_{10,9}$, which by (ii) are epimorphisms. Next, due to (iii), we have that $\tilde{\theta}_{(5, \ldots, 3)}=\theta_{5,4} \circ \theta_{4,3}$ is nonzero, since $\theta_{5,4}$ is an epimorphism, $\theta_{4,3}$ is a monomorphism and $5+5>6$, so by (ii) $\tilde{\theta}_{(5, \ldots, 3)}$ 
is a monomorphism. Hence, $\tilde{\theta}_{(5, \ldots, 2)}=\tilde{\theta}_{(5, \ldots, 3)} \circ \theta_{3,2}$ and $\tilde{\theta}_{(5, \ldots, 1)}=\tilde{\theta}_{(5, \ldots, 3)} \circ \tilde{\theta}_{(3, \ldots, 1)}$ are nonzero monomorphisms. Similarly, by (ii) and (iii), we have also that $\tilde{\theta}_{(6, \ldots, 3)}=\theta_{6,5} \circ$ $\tilde{\theta}_{(5, \ldots, 3)}$ and $\tilde{\theta}_{(7, \ldots, 3)}=\tilde{\theta}_{(7, \ldots, 5)} \circ \tilde{\theta}_{(5, \ldots, 3)}$ are nonzero, so they are epimorphisms $(5+3>5$ and $5+1>5)$. Analogously, $\tilde{\theta}_{(6, \ldots, 2)}=\theta_{6,5} \circ \tilde{\theta}_{(5, \ldots, 2)}$ is a nonzero monomorphism, since $3+3>5$. Consequently, $\tilde{\theta}_{(6, \ldots, 1)}=\tilde{\theta}_{(6, \ldots, 2)} \circ \theta_{2,1}$ is a nonzero monomorphism and $\tilde{\theta}_{(7, \ldots, 2)}=$ $\theta_{7,6} \circ \tilde{\theta}_{(6, \ldots, 2)}$ a nonzero epimorphisms (in the second case apply $3+1>3$ ). Finally, $\tilde{\theta}_{(9,4,3)}=$ $\theta_{9,4} \circ \theta_{4,3}$ is a nonzero map, since $5+3>6$. In consequence, there exist nonzero $u, v \in k$ such that $\tilde{\theta}_{(9,4,3)}=u \tilde{\theta}_{(9,8,3)}$ and $\tilde{\theta}_{(10,5,4)}=v \tilde{\theta}_{(10,9,4)}$, since $\operatorname{dim}_{k} \operatorname{Hom}_{\mathbb{X}}\left(T_{3}, T_{9}\right)$, $\operatorname{dim}_{k} \operatorname{Hom}_{\mathbb{X}}\left(T_{5}, T_{10}\right)=1$. Thus, due to $\tilde{\theta}_{(10,9,8,3)} \neq 0$, we have also $\tilde{\theta}_{(10,9,4,3)}, \tilde{\theta}_{(10,5,4,3)} \neq 0$.

Using the same type of arguments we show the assertion of the claim for the next 5 of the remaining $6(=35-11-18)$ pairs $(i, j)$. In particular, $\tilde{\theta}_{(8, \ldots, 2)}$ and $\tilde{\theta}_{(9,8,3,2)}$ are nonzero monomorphisms $(3+4>5$ and $3+3>4), \tilde{\theta}_{(10,9,8,3,2)}$ is a nonzero epimorphism $(3+1>$ $3), \tilde{\theta}_{(8, \ldots, 1)}=\tilde{\theta}_{(8, \ldots, 2)} \circ \theta_{2,1}$ and $\tilde{\theta}_{(9,8,3,2,1)}=\tilde{\theta}_{(9,8,3,2)} \circ \theta_{2,1}$ are nonzero monomorphisms. Due to the two equalities above involving scalars $u$ and $v$ respectively, it is clear that to complete the proof of the claim it remains only to show that $\tilde{\theta}_{(10,9,8,3,2,1)}=\tilde{\theta}_{(10,9,8,3,2)}$ 。 $\theta_{2,1} \neq 0$. Because $\theta_{2,1} \neq 0$, so

(iv): it suffices only to show that $\operatorname{Hom}_{\mathbb{X}}\left(T_{1}, K\right)=0$

where $K:=\operatorname{Ker} \tilde{\theta}_{(10,9,8,3,2)}$ is the kernel of the epimorphism $\tilde{\theta}_{(10,9,8,3,2)}: T_{2} \rightarrow T_{10}$.

$T$ is a tilting sheaf, hence $\left(T_{2}, T_{10}\right)$ is an exceptional pair and $K$ is an exceptional object as a result of a mutation of the exceptional pair $\left(T_{2}, T_{10}\right)$ (see [17, 4.2]), Moreover, we have $\operatorname{rk}(K)=3-1=2, \operatorname{deg}(K)=\operatorname{deg}\left(T_{2}\right)-\operatorname{deg}\left(T_{10}\right)=0$ and $\mu(K)=0$. Furthermore,

$$
\begin{aligned}
& \operatorname{eulf}\left(\left[T_{1}\right],[K]\right)=\operatorname{eulf}\left(\left[T_{1}\right],\left[T_{2}\right]\right)-\operatorname{eulf}\left(\left[T_{1}\right],\left[T_{10}\right]\right) \\
& =\operatorname{dim}_{k} \operatorname{Hom}_{\mathbb{X}}\left(T_{1}, T_{2}\right)-\operatorname{dim}_{k} \operatorname{Hom}_{\mathbb{X}}\left(T_{1}, T_{10}\right)=1-1=0 .
\end{aligned}
$$

Consequently, we have $\operatorname{dim}_{k} \operatorname{Hom}_{\mathbb{X}}\left(T_{1}, K\right)=\operatorname{dim}_{k} \operatorname{Ext}_{\mathbb{X}}^{1}\left(T_{1}, K\right)$, and by Serre duality we conclude

$$
\text { (v): } \quad \operatorname{dim}_{k} \operatorname{Hom}_{\mathbb{X}}\left(T_{1}, K\right)=\operatorname{dim}_{k} \operatorname{Ext}_{\mathbb{X}}^{1}\left(T_{1}, K\right)=\operatorname{dim}_{k} \operatorname{Hom}_{\mathbb{X}}\left(K, \tau_{\mathbb{X}}\left(T_{1}\right)\right) .
$$

Suppose that $\operatorname{Hom}_{\mathbb{X}}\left(T_{1}, K\right) \neq 0$. Then due to $3.4(*)$ we have $\mu\left(T_{1}\right)=\mu(K)=\frac{0}{1}$. By the formula 3.4(**) for the rank function we infer that either $\rho(K)=3$, or $\rho(K)=6$ and $\ell(K)=2$; additionally, $\rho\left(T_{1}\right)=6$ and $\ell\left(T_{1}\right)=1$. Notice that the first case is impossible, since then $T_{1}$ and $K$ belong to different tubes in the same slope, which are always $\mathrm{Hom}_{\mathbb{X}}$ orthogonal. Let now $\rho(K)=6$ and $\ell(K)=2 . K$ and $T_{1}$ belong to the same tube of the rank $6, T_{1}$ is a quasi-simple object, so $K$ lies on the ray starting at $T_{1}$, hence $\operatorname{dim}_{k} \operatorname{Hom}_{\mathbb{X}}\left(T_{1}, K\right)=$ 1. Then by (v), we have $\operatorname{dim}_{k} \operatorname{Hom}_{\mathbb{X}}\left(K, \tau_{\mathbb{X}}\left(T_{1}\right)\right)=1$, so $K$ lies on the coray ending with $\tau_{X}\left(T_{1}\right)$. But it easy to see that the minimum of the quasi-lengths $\ell(X)$, for the members $X$ of the intersection of this ray and this coray is equal to 6 , a contradiction. Therefore $\operatorname{Hom}_{\mathbb{X}}\left(T_{1}, K\right)=0$, so $\tilde{\theta}_{(10,9,8,3,2,1)} \neq 0$ due to (iv), and the claim is proved.

To finish the proof we define an algebra homomorphism $\psi: k Q \rightarrow E$. For each $(i, j) \in \Omega$, denote by $\theta_{j, i}^{\prime}$ the endomorphism $\theta_{j, i}^{\prime}:=w_{j} \circ \theta_{j, i} \circ p_{i}: T \rightarrow T$, where $p_{i}: T \rightarrow T_{i}$ is the canonical $i$ th projection and $w_{j}: T_{j} \rightarrow T$ the canonical $j$ th embedding. Then we set $\psi\left(\alpha_{1}\right)$ $:=\theta_{2,1}^{\prime}, \psi\left(\alpha_{2}\right):=\theta_{3,2}^{\prime}, \ldots, \psi\left(\alpha_{6}\right):=\theta_{7,6}^{\prime}, \psi\left(\gamma_{1}\right):=\theta_{8,3}^{\prime}, \psi\left(\gamma_{2}\right):=\theta_{9,4}^{\prime}, \psi\left(\gamma_{3}\right):=\theta_{10,5}^{\prime}$, $\psi\left(\beta_{1}\right):=u \theta_{9,8}^{\prime}, \psi\left(\beta_{2}\right):=v \theta_{10,9}^{\prime}$, where $u, v$ are as above. By the claim, the observation (i) and the fact that all the nonzero spaces $\operatorname{Hom}_{\mathbb{X}}\left(T_{i}, T_{j}\right)$ are one-dimensional, we infer that $\theta_{j, i}^{\prime} \in \operatorname{Im} \psi$ for every $(i, j) \in \Omega$, so $\operatorname{Im} \psi=E$. Moreover, $\psi(\Delta)=0$, since by assumption 
$\operatorname{Hom}_{\mathbb{X}}\left(T_{1}, T_{7}\right)=0$ and the equalities defining $u, v$ hold. In this way the proof is complete.

\subsection{0}

Before we pass to the proof Theorem 3.6 we briefly collect some necessary notions and facts. We notice first that by the very definition of $\mathcal{B}_{\mathcal{S}}$, the matrix $V$ (of the respective base change) with columns being the coordinate vectors of the consecutive members of $\mathcal{B}_{\mathcal{S}}$ with respect to $\mathcal{B}_{\mathcal{O}}$, and the matrix $G_{\mathcal{B}_{\mathcal{S}}}\left(=V^{t r} \cdot G_{\mathcal{B}_{\mathcal{O}}} \cdot V\right)$ of the Euler form with respect to $\mathcal{B}_{\mathcal{S}}$, have respectively the following forms:

$$
V:=\left[\begin{array}{rrrrrrrrrr}
1 & 0 & 0 & 0 & 0 & 0 & 0 & 0 & 0 & -1 \\
0 & -1 & 0 & 0 & 0 & 0 & 0 & 0 & 0 & 0 \\
0 & 0 & -1 & 0 & 0 & 0 & 0 & 0 & 0 & 0 \\
0 & 0 & 1 & -1 & 0 & 0 & 0 & 0 & 0 & 0 \\
0 & 0 & 0 & 0 & -1 & 0 & 0 & 0 & 0 & 0 \\
0 & 0 & 0 & 0 & 1 & -1 & 0 & 0 & 0 & 0 \\
0 & 0 & 0 & 0 & 0 & 1 & -1 & 0 & 0 & 0 \\
0 & 0 & 0 & 0 & 0 & 0 & 1 & -1 & 0 & 0 \\
0 & 0 & 0 & 0 & 0 & 0 & 0 & 1 & -1 & 0 \\
0 & 1 & 0 & 1 & 0 & 0 & 0 & 0 & 1 & 1
\end{array}\right], \quad G_{\mathcal{B} S}:=\left[\begin{array}{rrrrrrrrrr}
1 & 1 & 0 & 1 & 0 & 0 & 0 & 0 & 1 & 1 \\
0 & 1 & 0 & 0 & 0 & 0 & 0 & 0 & 0 & 0 \\
0 & 0 & 1 & 0 & 0 & 0 & 0 & 0 & 0 & 0 \\
0 & 0 & -1 & 1 & 0 & 0 & 0 & 0 & 0 & 0 \\
0 & 0 & 0 & 0 & 1 & 0 & 0 & 0 & 0 & 0 \\
0 & 0 & 0 & 0 & -1 & 1 & 0 & 0 & 0 & 0 \\
0 & 0 & 0 & 0 & 0 & -1 & 1 & 0 & 0 & 0 \\
0 & 0 & 0 & 0 & 0 & 0 & -1 & 1 & 0 & 0 \\
0 & 0 & 0 & 0 & 0 & 0 & 0 & -1 & 1 & 0 \\
-1 & 0 & 0 & 0 & 0 & 0 & 0 & 0 & 0 & 0
\end{array}\right] .
$$

Moreover, the matrices $\underline{\mathrm{rk}}_{\mathcal{B}_{\mathcal{S}}}$ and $\underline{\mathrm{rk}}_{\mathcal{B}_{\mathcal{S}}}$ of the rank and degree homomorphisms rk, deg : $\mathrm{K}_{0}(\mathbb{X}) \rightarrow \mathbb{Z}$ with respect to the basis $\mathcal{B}_{\mathcal{S}}$ (and the standard basis $\{1\}$ of $\mathbb{Z}$ ) are given as follows:

$$
\underline{\mathrm{rk}}_{\mathcal{B}_{\mathcal{S}}}=[1,0, \ldots, 0], \quad \underline{\operatorname{deg}}_{\mathcal{B}_{\mathcal{S}}}=[0,3,2,2,1,1,1,1,1,6] .
$$

In our considerations a crucial role is played by a pair of autoequivalences $R$ and $S$ of the derived category $\mathcal{D}^{b} \operatorname{coh}(\mathbb{X})$, defined as tubular mutations (see [16]). They are nicely controlled on the level of the Grothendieck group. We have the following commutative diagram:

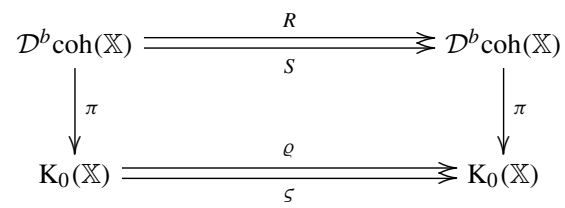

where $\pi: \mathcal{D}^{b} \operatorname{coh}(\mathbb{X}) \longrightarrow \mathrm{K}_{0}\left(\mathcal{D}^{b} \operatorname{coh}(\mathbb{X})\right) \cong \mathrm{K}_{0}(\mathbb{X})$ denotes the canonical passage to the Grothendieck class and $\varrho, \varsigma$ are the $\mathbb{Z}$-linear automorphisms of $\mathrm{K}_{0}(\mathbb{X})$ induced by $R, S$, respectively $\left(\varrho\right.$ and $\varsigma$ preserve the Euler form eulf $: \mathrm{K}_{0}(\mathbb{X})^{2} \rightarrow \mathbb{Z}$ ). The matrices of the maps $\varrho, \varsigma$ can be easily computed from the very definition of the functors (see $[3,7,14,16]$ for the details). In fact, we have the following identification showing the actual meaning of the two first members of the triple $(\dot{R}, \dot{S}, U)$ of matrices, defined in Section 2.2.

Lemma 3.11 The matrices of the $\mathbb{Z}$-linear transformations $\varrho, \varsigma: \mathrm{K}_{0}(\mathbb{X}) \rightarrow \mathrm{K}_{0}(\mathbb{X})$ in the basis $\mathcal{B}_{\mathcal{S}}$ coincide with $\dot{R}$ and $\dot{S}$, respectively.

\subsection{2}

Restricted to $\operatorname{coh}(\mathbb{X}), R$ in contrast to $S$, is no longer an autoequivalence. Nevertheless, some special restrictions of $R$ and $S$ yield isomorphisms

$$
\begin{aligned}
& S: \mathcal{C}_{q} \stackrel{\sim}{\longrightarrow} \mathcal{C}_{q+1} \quad \text { for all } \quad q \in \mathbb{Q} \\
& R: \mathcal{C}_{q} \stackrel{\sim}{\longrightarrow} \mathcal{C}_{\frac{q}{1+q}} \quad \text { for all } \quad 0 \leq q \leq \infty
\end{aligned}
$$


of categories. As a result, for any $q \in \mathbb{Q}$ there exists a unique canonical sequence of powers of $R$ and $S$ such that their composition called telescoping functor and given by the formula $(* *)$ $\Phi_{q, \infty}:= \begin{cases}S^{c_{1}} \circ R^{c_{2}} \circ S^{c_{3}} \circ \cdots \circ S^{c_{m-1}} \circ R^{c_{m}} & \text { if } m \in 2 \mathbb{N}, \\ S^{c_{1}} \circ R^{c_{2}} \circ \cdots \circ R^{c_{m-1}} \circ S^{c_{m}-1} \circ R & \text { if } m \notin 2 \mathbb{N},\end{cases}$

where $q=\left[c_{1} ; \ldots, c_{m}\right]$ is the continued fraction presentation of $q \in \mathbb{Q}$, yields an isomorphism $\mathcal{C}_{\infty} \cong \mathcal{C}_{q}$. (We set additionally $\Phi_{\infty, \infty}:=\mathrm{Id}$ ).

For any $q \in \overline{\mathbb{Q}}$, we denote by $\phi_{q, \infty}$ the automorphism of $\mathrm{K}_{0}(\mathbb{X})$ controlling $\Phi_{q, \infty}$, which is defined by the formula (**) with the $R$ and $S$ replaced by $\varrho$ and $\varsigma$, respectively. It is clear that we have

$(* * *)$

$$
\phi_{q, \infty} \circ \pi=\pi \circ \Phi_{q, \infty}
$$

and that the matrix of $\phi_{q, \infty}$ in the basis $\mathcal{B}_{\mathcal{S}}$ is equal to the matrix $\dot{\Phi}_{q, \infty} \in \mathbb{M}_{10}(\mathbb{Z})$, where $\dot{\Phi}_{q, \infty}$ is defined by the formula (**) now with the functors $R$ and $S$ replaced by the matrices $\dot{R}$ and $\dot{S}$, respectively. (We set additionally $\phi_{\infty, \infty}:=\operatorname{id}_{\mathrm{K}_{0}(\mathbb{X})}$ and $\dot{\Phi}_{\infty, \infty}:=I_{10}$ ).

Proof of Theorem 3.6. We construct a family $\tilde{T}:=\left\{T_{i}\right\}_{i \in[10]}$ of quasi-simple sheaves which will serve for the indecomposable direct summands of a tilting sheaf $T$ with the slopes prescribed by $\mu(T)=\left(0, \frac{1}{3}, \frac{2}{5}, \frac{1}{2}, \frac{3}{5}, \frac{2}{3}, 1, \frac{1}{2}, \frac{2}{3}, 1\right)$, and we show first that $\tilde{T}$ has all the properties from (a) and (b). We use for this aim the telescoping functors $\Phi_{q, \infty}$, for $q \in$ $\left\{0, \frac{1}{3}, \frac{2}{5}, \frac{1}{2}, \frac{3}{5}, \frac{2}{3}, 1\right\}$. The respective sequences of exponents of powers of the functors $R$ and $S$ constituting $\Phi_{q, \infty}$ are completely determined by the continued fraction presentation of the rational numbers $\frac{0}{1}, \frac{1}{3}, \frac{2}{5}, \frac{1}{2}, \frac{3}{5}, \frac{2}{3}, \frac{1}{1}$, which are equal [0], [0;3], [0;2, 2], [0;2], [0;1, 1, 2], $[0 ; 1,2]$ and [1], respectively (see $(* *))$.

We set: $T_{1}:=\left(S^{-1} \circ R\right)\left(\mathcal{S}_{3,5}\right), T_{2}:=R^{3}\left(\mathcal{S}_{3,1}\right), T_{3}:=\left(R^{2} \circ S \circ R\right)\left(\mathcal{S}_{3,1}\right)$,

$$
\begin{aligned}
& T_{4}:=R^{2}\left(\mathcal{S}_{1,0}\right), T_{5}:=\left(R \circ S \circ R^{2}\right)\left(\mathcal{S}_{3,0}\right), T_{6}:=(R \circ S \circ R)\left(\mathcal{S}_{3,0}\right), \\
& T_{7}:=R\left(\mathcal{S}_{3,1}\right), T_{8}:=R^{2}\left(\mathcal{S}_{2,0}\right), T_{9}:=(R \circ S \circ R)\left(\mathcal{S}_{3,4}\right), T_{10}:=R\left(\mathcal{S}_{3,5}\right) .
\end{aligned}
$$

Note that by the very construction $\underline{\mu}(T)$, where $T=\bigoplus_{i \in[10]} T_{i}$, has the required form.

Now we determine the coordinate vectors $\left[T_{i}\right]_{\mathcal{B}_{\mathcal{S}}} \in \mathbb{Z}^{10}$ of $\left[T_{i}\right] \in \mathrm{K}_{0}(\mathbb{X})$, for $i \in[10]$, with respect to the basis $\mathcal{B}_{\mathcal{S}}$. We start by computing (if necessary) the following products of the matrices: $\Xi^{\prime}(1):=\dot{S}^{-1} \dot{R}, \Xi^{\prime}(2):=\dot{R}^{3}, \Xi^{\prime}(3):=\dot{R}^{2} \dot{S} \dot{R}, \Xi^{\prime}(4):=\dot{R}^{2}, \Xi^{\prime}(5):=$ $\dot{R} \dot{S} \dot{R}^{2}, \Xi^{\prime}(6):=\dot{R} \dot{S} \dot{R}, \Xi^{\prime}(7):=\dot{R}$ in $\mathbb{M}_{10}(\mathbb{Z})$, being in fact the matrices of the automorphisms $\phi_{q, \infty}$ with respect to $\mathcal{B}_{\mathcal{S}}$, for $q=0, \frac{1}{3}, \frac{2}{5}, \frac{1}{2}, \frac{3}{5}, \frac{2}{3}, 1$ (see Section 2.2 for the definition of $\dot{R}$ and $\dot{S}$ ). Now due to (*), applying the definition of $\mathcal{B}_{\mathcal{S}}$, we have:

$$
\begin{aligned}
& {\left[T_{1}\right]_{\mathcal{B}_{\mathcal{S}}}=\Xi^{\prime}(1)^{\langle 9\rangle},\left[T_{2}\right]_{\mathcal{B}_{\mathcal{S}}}=\Xi^{\prime}(2)^{\langle 5\rangle},\left[T_{3}\right]_{\mathcal{B}_{\mathcal{S}}}=\Xi^{\prime}(3)^{\langle 5\rangle},\left[T_{4}\right]_{\mathcal{B}_{\mathcal{S}}}=\Xi^{\prime}(4)^{\langle 10\rangle}-\Xi^{\prime}(4)^{\langle 2\rangle},} \\
& {\left[T_{5}\right]_{\mathcal{B}_{\mathcal{S}}}=\Xi^{\prime}(5)^{\langle 10\rangle}-\left(\Xi^{\prime}(5)^{\langle 5\rangle}+\ldots+\Xi^{\prime}(5)^{\langle 9\rangle}\right),\left[T_{6}\right]_{\mathcal{B}_{\mathcal{S}}}=\Xi^{\prime}(6)^{\langle 10\rangle}-\left(\Xi^{\prime}(6)^{\langle 5\rangle}+\ldots+\Xi^{\prime}(6)^{\langle 9\rangle}\right),} \\
& {\left[T_{7}\right]_{\mathcal{B}_{\mathcal{S}}}=\Xi^{\prime}(7)^{\langle 5\rangle},\left[T_{8}\right]_{\mathcal{B}_{\mathcal{S}}}=\Xi^{\prime}(4)^{\langle 10\rangle}-\Xi^{\prime}(4)^{\langle 3\rangle}-\Xi^{\prime}(4)^{\langle 4\rangle},\left[T_{9}\right]_{\mathcal{B}_{\mathcal{S}}}=\Xi^{\prime}(6)^{\langle 8\rangle},\left[T_{10}\right]_{\mathcal{B}_{\mathcal{S}}}=\Xi^{\prime}(7)^{\langle 9\rangle} .}
\end{aligned}
$$

Once we already know the explicit values of all 10 expressions above we find out that the matrix $\Theta_{\mathcal{B}_{\mathcal{S}}}:=\left[\left[T_{1}\right]_{\mathcal{B}_{\mathcal{S}}}|\ldots|\left[T_{10}\right]_{\mathcal{B}_{\mathcal{S}}}\right] \in \mathbb{M}_{10}(\mathbb{Z})$ has really the required form $\Theta_{\mathcal{B}_{\mathcal{S}}}=\Theta$. Moreover, we have $\left[\mathrm{rk}\left(T_{i}\right)\right]_{i \in[10]}=\underline{\mathrm{rk}}_{\mathcal{B}_{S}} \cdot \Theta=[1,3,5,6,5,3,1,4,3,1]$. The shape of $\underline{\mu}(T)$ and $\underline{\mathrm{rk}}(T)$ implies that $\underline{\operatorname{deg}(T)}$ has also the form as in (a).

Next we prove that $T=\bar{\bigoplus}_{i \in[10]} T_{i}$ is a tilting sheaf. Observe that the family $\tilde{T}$ of quasi simple sheaves satisfies the condition (b) from Lemma 3.8 (one has to consider only the pairs $(i, j)=(4,8),(6,9),(7,10))$. Next we have to determine the matrix $G_{\tilde{T}}:=$ [eulf $\left.\left(\left[T_{i}\right],\left[T_{j}\right]\right)\right]_{i, j \in[10]}$ consisting of the values of the Euler form on all the pairs $\left(T_{i}, T_{j}\right)$. A direct calculation via the formula $G_{\tilde{T}}=\left(\Theta_{\mathcal{B}_{\mathcal{S}}}\right)^{t r} \cdot G_{\mathcal{B}_{\mathcal{S}}} \cdot \Theta_{\mathcal{B}_{\mathcal{S}}}$ yields the equality $G_{\tilde{T}}=$ 
$C_{\Lambda}$. Then a case by case verification of positivity of the respective coefficients of $C_{\Lambda}$ shows that also the condition (a) from Lemma 3.8 holds for $\tilde{T}$. Consequently, $T$ is a tilting sheaf and $C_{E}=C_{\Lambda}$, where $E=\operatorname{End}_{\mathbb{X}}(T)$.

To complete the proof note that $\Lambda \cong E$, by Proposition 3.9. Notice that the uniqueness, up to isomorphism, of $T$ follows from the fact that exceptional sheaves are determined uniquely by their Grothendieck classes $[14,17]$. In this way our proof is finished.

\section{$4 \Lambda$-Modules Versus Coherent Sheaves, Applications}

From now on we assume that $T$ is precisely as in the Theorem 3.6 and the isomorphism $\Lambda \cong$ $\operatorname{End}_{\mathbb{X}}(T)$ is given by the induced homomorphism $\bar{\psi}$, defined in the proof of Proposition 3.9. Moreover, all basic vectors $\theta_{j, i} \in \operatorname{Hom}_{\mathbb{X}}\left(T_{i}, T_{j}\right)$, for $(i, j) \in \Omega$, are chosen in a compatible way, i.e. $\theta_{j, i}$, for $(i, j) \in \Omega^{\prime}$, correspond to images of the arrows from $Q_{1}$ via $\bar{\psi}$ and satisfy the relations defining $\Delta$, whereas the remaining $\theta_{j, i}$, for $(i, j) \in \Omega \backslash \Omega^{\prime}$, are given as the respective compositions of the previous ones.

Under the setting above we have the following comparison result.

Theorem 4.1 (a) The full right derived functor $\Psi:=\mathbb{R} \operatorname{Hom}_{\mathbb{X}}(T,-): \mathcal{D}^{b} \operatorname{coh}(\mathbb{X}) \rightarrow$ $\mathcal{D}^{b} \bmod (\Lambda)$ for the functor $\operatorname{Hom}_{\mathbb{X}}(T,-): \operatorname{coh}(\mathbb{X}) \rightarrow \bmod (\Lambda)$, yields a triangle equivalence $\mathcal{D}^{b} \operatorname{coh}(\mathbb{X}) \simeq \mathcal{D}^{b} \bmod (\Lambda)$. In particular, the mapping $[\mathcal{F}] \mapsto\left[\operatorname{Hom}_{\mathbb{X}}(T, \mathcal{F})\right]-$ $\left[\operatorname{Ext}_{\mathbb{X}}^{1}(T, \mathcal{F})\right]$, for $\mathcal{F}$ in $\operatorname{coh}(\mathbb{X})$, yields an isomorphism $\mathrm{K}_{0}(\mathbb{X}) \cong \mathrm{K}_{0}(\Lambda)$ of the Grothendieck groups.

(b) Both, $\mathcal{B}_{T}:=\left\{\left[T_{i}\right]\right\}_{i \in[10]}$ and $\mathcal{B}_{\mathcal{E}}:=\left\{\left[S_{i}\right]\right\}_{i \in[10]}$, where $\left\{S_{i}\right\}_{i \in[10]}$ is a full list of standard simple $\Lambda$-modules, form bases of $\mathrm{K}_{0}(\mathbb{X})=\mathrm{K}_{0}(\Lambda)$. The base change matrix $A_{\mathcal{B}_{\mathcal{E}}, \mathcal{B}_{T}}:=\left[\left[T_{1}\right]_{\mathcal{B}_{\mathcal{E}}}|\ldots|\left[T_{10}\right]_{\mathcal{B}_{\mathcal{E}}}\right]$ is equal to $C_{\Lambda}$. Moreover,

$$
\underline{\mathrm{rk}}_{\mathcal{B}_{\mathcal{E}}}=[1,2,2,1,-1,-2,-1 ;-1,-2,-1], \quad \underline{\operatorname{deg}}_{\mathcal{B}_{\mathcal{E}}}=[0,1,1,1,0,-1,-1 ; 0,-1,-1]
$$

where $\underline{\mathrm{rk}}_{\mathcal{B}_{\mathcal{E}}}$ and $\underline{\mathrm{rk}}_{\mathcal{B}_{\mathcal{E}}}$ denote matrices of the forms $\mathrm{rk}$, deg $: \mathrm{K}_{0}(\mathbb{X}) \rightarrow \mathbb{Z}$ with respect to the basis $\mathcal{B}_{\mathcal{E}}$, respectively.

Proof Follows immediately from Theorem 3.6 and the shape of the respective matrices (see also the general result concerning the tilting procedure [7]).

As a straightforward consequence we obtain also an explanation of the actual role of the third matrix from the triple $(\dot{R}, \dot{S}, U)$, defined in Section 2.2.

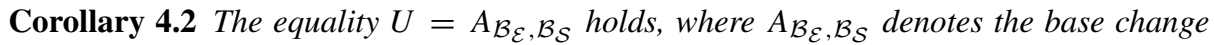
matrix with columns being the coordinate vectors of the consecutive elements of $\mathcal{B}_{\mathcal{S}}$ with respect to $\mathcal{B}_{\mathcal{E}}$.

Proof By Theorems 4.1(b) and 3.6(b), we have $A_{\mathcal{B}_{\mathcal{E}}, \mathcal{B}_{\mathcal{S}}}=A_{\mathcal{B}_{\mathcal{E}}, \mathcal{B}_{T}} \cdot A_{\mathcal{B}_{T}, \mathcal{B}_{\mathcal{S}}}=C_{\Lambda} \cdot \Theta^{-1}$, where $A_{\mathcal{B}_{T}, \mathcal{B}_{\mathcal{S}}}$ is a base change matrix formed by the coordinates of the consecutive members of $\mathcal{B}_{\mathcal{S}}$ with respect to $\mathcal{B}_{T}$. An easy check shows that the right hand side product of matrices just coincides with $U$. 


\section{3}

Due to Theorem 4.1 we can identify $\Lambda$-modules with the corresponding sheaves (or their copies shifted by the translation functor [1] in the derived category). Note that we have in our disposal the notion of rank and degree for $\Lambda$-modules, hence also of the slope (see Section 4.1(b) for the explicit formulas). We explain below details of this identification.

Now let $\operatorname{coh}_{+}(\mathbb{X})($ respectively coh_$(\mathbb{X}))$ be the full subcategory of $\operatorname{vect}(\mathbb{X})$ formed by all vector bundles whose indecomposable summands $\mathcal{F}$ satisfy the condition $\operatorname{Ext}_{\mathbb{X}}^{1}(T, \mathcal{F})=0$ (respectively $\left.\operatorname{Hom}_{\mathbb{X}}(T, \mathcal{F})=0\right)$. Further, we denote by $\bmod _{+}(\Lambda)\left(\operatorname{respectively} \bmod _{0}(\Lambda)\right.$, $\left.\bmod _{-}(\Lambda)\right)$ the full subcategories of $\bmod (\Lambda)$ formed by all $\Lambda$-modules whose indecomposable summands have positive rank (respectively zero rank, negative rank). Finally, $\operatorname{coh}_{\geq}(\mathbb{X})$ $\left(\right.$ respectively, $\bmod _{\geq}(\Lambda)$ ) denotes the additive closure of $\operatorname{coh}_{+}(\mathbb{X}) \cup \operatorname{coh}_{0}(\mathbb{X})$ (respectively, $\left.\bmod _{+}(\Lambda) \cup \bmod _{0}(\Lambda)\right)$.

Then under the equivalence $\Psi: \mathcal{D}^{b} \operatorname{coh}(\mathbb{X}) \stackrel{\simeq}{\rightarrow} \mathcal{D}^{b} \bmod (\Lambda)$

- $\operatorname{coh}_{+}(\mathbb{X})$ corresponds to $\bmod _{+}(\Lambda)$ by means of $\mathcal{F} \mapsto \operatorname{Hom}_{\mathbb{X}}(T, \mathcal{F})$,

- $\operatorname{coh}_{0}(\mathbb{X})$ corresponds to $\bmod _{0}(\Lambda)$ by means of $\mathcal{F} \mapsto \operatorname{Hom}_{\mathbb{X}}(T, \mathcal{F})$,

- $\quad$ coh_(X) $[1]$ corresponds to $\bmod _{-}(\Lambda)$ by means of $\mathcal{F}[1] \mapsto \operatorname{Ext}_{\mathbb{X}}^{1}(T, \mathcal{F})$.

The structure of the Auslander-Reiten quiver of $\bmod (\Lambda)$ and the shape of its components, for the algebra $\Lambda$ of tubular type can be now derived alternatively from the description of the Auslander-Reiten quiver of $\operatorname{coh}(\mathbb{X})$ by applying tilting theory [14, Theorem 5.7]. In particular, one can precisely reconstruct the one-parameter families of tubes forming $\mathcal{T}$ from those for $\operatorname{coh}(\mathbb{X})$ and present $\mathcal{T}$ in a form $\mathcal{T}=\left(\mathcal{T}^{q}\right)_{q \in \overline{\mathbb{Q}}}$, slightly different as originally. Namely, $\Gamma_{\Lambda}$ consists of the components described as follows:

- for each $q \in \overline{\mathbb{Q}}$ with $q>1$ or $q<0$ a family $\left(\mathcal{T}_{\lambda}^{q}\right)_{\lambda \in \mathbb{X}}=\left(\mathcal{T}_{\lambda}^{q}(\Lambda)\right)_{\lambda \in \mathbb{X}}$ of stable tubes, being the images of $\left(\mathcal{T}_{\lambda}^{q}(\mathbb{X})\right)_{\lambda \in \mathbb{X}}$ (in the second case in fact of $\left(\mathcal{T}_{\lambda}^{q}(\mathbb{X})[1]\right)_{\lambda \in \mathbb{X}}$ in $\operatorname{coh}(\mathbb{X})[1])$, whose ranks equal the weights of $\lambda$;

- a family $\left(\mathcal{T}_{\lambda}^{1}\right)_{\lambda \in \mathbb{X}}=\left(\mathcal{T}_{\lambda}^{1}(\Lambda)\right)_{\lambda \in \mathbb{X}}$ of tubes such that $\mathcal{T}_{\lambda}^{1}$, for $\lambda \in \mathbb{X} \backslash\left\{\lambda_{3}\right\}$, are stable tubes, being the images of $\left(\mathcal{T}_{\lambda}^{1}(\mathbb{X})\right)_{\lambda \in \mathbb{X} \backslash\left\{\lambda_{3}\right\}}$, whose ranks equal the weights of $\lambda$, and a tube $\mathcal{T}_{\lambda_{3}}^{1}$ is obtained from the stable tube $\mathcal{T}_{\lambda_{3}}^{1}(\mathbb{X})$ of rank 6 by deletion of 2 corays ending at the vertex $\tau_{\mathbb{X}} T_{7}$ and $\tau_{\mathbb{X}} T_{10}$, respectively $\left(\mathcal{T}_{\lambda_{3}}^{1}\right.$ contains 2 projective $\Lambda$ modules $P(7)$ and $P(10)$ );

- a family $\left(\mathcal{T}_{\lambda}^{0}\right)_{\lambda \in \mathbb{X}}=\left(\mathcal{T}_{\lambda}^{0}(\Lambda)\right)_{\lambda \in \mathbb{X}}$ of tubes such that $\mathcal{T}_{\lambda}^{0}$, for $\lambda \in \mathbb{X} \backslash\left\{\lambda_{3}\right\}$, are stable tubes, being the images of $\left(\mathcal{T}_{\lambda}^{0}(\mathbb{X})\right)_{\lambda \in \mathbb{X} \backslash\left\{\lambda_{3}\right\}}$ (in fact of $\left(\mathcal{T}_{\lambda}^{0}(\mathbb{X})[1]\right)_{\lambda \in \mathbb{X}}$ ), whose ranks equal the weights of $\lambda$, and a tube $\mathcal{T}_{\lambda_{3}}^{0}$ is obtained from the stable tube $\mathcal{T}_{\lambda_{3}}^{0}(\mathbb{X})$ of rank 6 by deletion of the ray starting at the vertex $T_{1}$, (in fact $T_{1}[1] ; \mathcal{T}_{\lambda_{3}}^{0}$ contains the injective $\Lambda$-module $I(1)$ );

- the preprojective and preinjective components $\mathcal{P}$ and $\mathcal{Q}$ are formed by the images of sheaves $\mathcal{F}$ from the tube families $\mathcal{T}^{q}(\mathbb{X})$, for $0 \leq \mu(\mathcal{F}) \leq 1$, such that $\operatorname{Ext}_{\mathbb{X}}^{1}(T, \mathcal{F})=0$ and $\operatorname{Hom}_{\mathbb{X}}(T, \mathcal{F})=0$, respectively.

Notice that the objects from the preprojective component $\mathcal{P}$ and the tubes $\left(\mathcal{T}_{\lambda}^{q}\right)_{\lambda \in \mathbb{X}}$ with $1 \leq q<\infty$ (respectively, from the preinjective component $\mathcal{Q}$ and the tubes $\left(\mathcal{T}_{\lambda}^{q}\right)_{\lambda \in \mathbb{X}}$ with $q \leq 0)$ form the category $\bmod _{+}(\Lambda)$ (respectively, $\left.\bmod _{-}(\Lambda)\right)$. Moreover, the objects from $\left(\mathcal{T}_{\lambda}^{\infty}\right)_{\lambda \in \mathbb{X}}$ form the subcategory $\bmod _{0}(\Lambda)$. 


\section{4}

Now we briefly compare the description of tube families in $\Gamma_{\Lambda}$ given above with the original one (see Section 3.3).

An indecomposable $\Lambda$-module $M$ lies in a tube $\mathcal{T}_{\lambda}^{q}$, for $\lambda \in \mathbb{X}$, of the Auslander-Reiten quiver $\Gamma_{\Lambda}$ of $\bmod (\Lambda)$ if and only if the equality $\mu(M)=q$ holds (can be immediately verified once we know $\operatorname{dim}_{k} M$ ). On other hand for a fixed $\gamma=\frac{\gamma_{\infty}}{\gamma_{0}} \in \overline{\mathbb{Q}}_{0}$ the dimension vectors of all $\Lambda$-modules from the tubes in the family $\mathcal{T}_{(\gamma)}$ have index $\gamma$ (see [19] for the precise definition). Recall that for each homogeneous tube they form the set $\mathbb{N}_{1} \cdot h_{\gamma}$ with $h_{\gamma}:=$ $\gamma_{0} h_{0}+\gamma_{\infty} h_{\infty}$ (in particular, $h_{1}=h_{0}+h_{\infty}$ ), where $h_{0}=[1,2,3,3,2,1,0 ; 2,1,0], h_{\infty}=$ $[0,1,2,3,3,2,1 ; 2,2,1] \in \mathbb{Z}^{10}$ are the standard generators of the radical spaces for the tame concealed algebras $\Lambda_{0}$ and $\Lambda_{\infty}$, respectively. (Note that $\operatorname{gcd}\left\{\left(h_{\gamma}\right)_{i}: i \in Q_{0}\right\}=1$, since we always assume that $\gamma_{\infty}, \gamma_{0} \in \mathbb{N}$ are coprime). Therefore, the number $q \in \overline{\mathbb{Q}}$, for $\gamma \in \overline{\mathbb{Q}}_{0}$, such that the full subcategories of $\bmod (\Lambda)$ formed by indecomposables from $\mathcal{T}_{(\gamma)}$ and $\mathcal{T}^{q}$ coincide, is determined by the equality $q=\mu\left(h_{\gamma}\right)$. Consequently, we infer that

$$
q=q(\gamma):=\frac{\gamma_{0}}{\gamma_{0}-\gamma_{\infty}}
$$

(see Section 2.2), $\operatorname{since} \operatorname{rk}\left(h_{0}\right)=\underline{\mathrm{rk}}_{\mathcal{B}_{\mathcal{E}}} \cdot h_{0}^{\text {tr }}=6, \operatorname{rk}\left(h_{\infty}\right)=\underline{\mathrm{rk}}_{\mathcal{B}_{\mathcal{E}}} \cdot h_{\infty}^{t r}=-6$ and $\operatorname{deg}\left(h_{0}\right)=$ $\underline{\operatorname{deg}}_{\mathcal{B}_{\mathcal{E}}} \cdot h_{0}^{\text {tr }}=6, \operatorname{deg}\left(h_{\infty}\right)=\underline{\operatorname{deg}}_{\mathcal{B}_{\mathcal{E}}} \cdot h_{\infty}^{\text {tr }}=0$. We have the following.

Lemma 4.5 The mapping $\gamma \mapsto q(\gamma)$ and its inverse $q \mapsto \gamma(q)$, given for $q=\frac{a}{b}$, with $a \in \mathbb{Z}$ and $b \in \mathbb{N}$ coprime, by the formula $\gamma(q)=\frac{a-b}{a}$, yield a bijection $\overline{\mathbb{Q}}_{0}^{\infty} \longleftrightarrow \overline{\mathbb{Q}}^{\prime}:=$ $\overline{\mathbb{Q}} \backslash(0,1)$, which translate the index of a family of tubes into its slope, and conversely. In particular, we have $\gamma(1)=0, \gamma(\infty)=1$ and $\gamma(0)=\infty$.

As a result of the translation formulas and Theorem 4.1 we obtain also a precise description of the quasi-simple objects in $\tilde{\mathcal{S}}$ in terms of sheaves. To formulate it we use the intuitively clear notation: if for $\mathcal{F}$ in $\operatorname{coh}(\mathbb{X})$ the object $M:=\Psi(\mathcal{F}[m])$ from $\mathcal{D}^{b} \bmod (\Lambda)$ belongs to $\bmod (\Lambda)$, where $m \in\{0,1\}$, then by $\widehat{\Psi}(\mathcal{F}[m])$ we always mean the $R$-module $\widehat{\Psi}(\mathcal{F}[m]):=\hat{M}$. Moreover, for any $q \in \overline{\mathbb{Q}}$ and $i \in[3]$, we denote by $\mathcal{F}_{q, i, j}:=\tau_{\mathbb{X}}^{-j} \mathcal{F}_{q, i, 0}$, $j \in \mathbb{Z}_{p_{i}}$, the "consecutive" quasi-simple sheaves in the exceptional tube of the rank $p_{i}$ in the family $\mathcal{T}^{q}(\mathbb{X})$, where $p_{1}=2, p_{2}=3, p_{3}=6$ (in the moment the choice of $\mathcal{F}_{q, i, 0}$ is not important, cf. Section 4.7).

Proposition 4.6 Let $\gamma \in \mathbb{Q}_{0}$ and $i \in[3]$ be a pair such that $(q, i) \neq(0,3)$. Then for any $s \in \mathbb{Z}_{p_{i}}$ we have an isomorphism $M_{i, s}(\gamma) \cong \widehat{\Psi}\left(\mathcal{F}_{(q(\gamma), i, \sigma(s))}\right)$, if $\gamma \leq 1$, respectively, $M_{i, s}(\gamma) \cong \widehat{\Psi}\left(\mathcal{F}_{(q(\gamma), i, \sigma(s))}[1]\right)$, if $\gamma>1$, in the category $\tilde{\mathcal{S}}$, where $\sigma=\sigma_{\gamma, i}$ is some cyclic permutation of $\mathbb{Z}_{p_{i}}$.

Proof Follows by the information from Sections 4.3, 4.4 and the final part of Section 3.3.

\section{7}

From now on we assume that for any $q \in \overline{\mathbb{Q}}$ and any pair $(i, j)$, with $i \in[3]$ and $j \in \mathbb{Z}_{p_{i}}$, the sheaves $\mathcal{F}_{q, i, j}$ are precisely equal to the images of the simple torsion sheaves $\mathcal{S}_{i, j}$ via the 
telescoping functor $\Phi_{q, \infty}$, i.e. $\mathcal{F}_{q, i, j}:=\Phi_{q, \infty}\left(\mathcal{S}_{i, j}\right)$. (Clearly, $\left.\mathcal{F}_{\infty, i, j}=\mathcal{S}_{i, j}\right)$. Moreover, for each pair $(\gamma, i) \in \mathbb{Q}_{0} \times[3],(\gamma, i) \neq(0,3)$, the indexing of the quasi-simple objects $M_{i, s}(\gamma)$, for $s \in \mathbb{Z}_{p_{i}}$, in the mouth of the corresponding tube in the category $\tilde{\mathcal{S}}$ is such that $\sigma_{\gamma, i}$ is the identity permutation.

Proof of Theorem 2.3. For $q \in \overline{\mathbb{Q}}^{\prime}$ and for the pair $(i, s)$, with $i \in[3]$ and $s \in \mathbb{Z}_{p_{i}}$, we set $X_{q, i, s}:=\Psi\left(\mathcal{F}_{q, i, s}\right)=\operatorname{Hom}\left(T, \mathcal{F}_{q, i, s}\right)$, if $q \geq 1$ and $(q, i, s) \neq(1,3,0),(1,3,4)$, and $X_{q, i, s}:=\Psi\left(\mathcal{F}_{q, i, s}[1]\right)=\operatorname{Ext}_{\mathbb{X}}^{1}\left(T, \mathcal{F}_{q, i, s}\right)$, if $q \leq 0$ and $(q, i, s) \neq(0,3,5)$. Then clearly the sets $\left\{X_{q, i, s}\right\}_{s \in \mathbb{Z}_{p_{i}}}, i \in[3]$ (with special treatment of the cases $(q, i)=(1,3),(0,3)$; cf. $L^{1}, L^{0}$ in Section 6), form full lists of the mouth $\Lambda$-modules in the consecutive exceptional tubes from the family $\mathcal{T}^{q}=\left(\mathcal{T}_{\lambda}^{q}(\Lambda)\right)_{\lambda \in \mathbb{X}}$, for a fixed $q \in \overline{\mathbb{Q}}^{\prime}$. To prove the assertion of Theorem 2.3 we determine the dimension vectors $\underline{\operatorname{dim}_{k}} X_{q, i, s}$ using the formula $3.12(* * *)$ (cf. [3]).

We know that $\left[\mathcal{F}_{q, i, s}\right]=\varphi_{q, \infty}\left(\left[\mathcal{S}_{i, s}\right]\right)$ in $\mathrm{K}_{0}(\mathbb{X})$, so $\left[\mathcal{F}_{q, i, s}\right]_{\mathcal{B}_{\mathcal{S}}}=\dot{\Phi}_{q, \infty} \cdot\left[\mathcal{S}_{i, s}\right]_{\mathcal{B}_{\mathcal{S}}}$. Hence, setting $\ddot{\Phi}_{q, \infty}:=U \cdot \ddot{\Phi}_{q, \infty}$, due to Corollary 4.2 we have $\left[\mathcal{F}_{q, i, s}\right]_{\mathcal{B}_{\mathcal{E}}}=\ddot{\Phi}_{q, \infty} \cdot\left[\mathcal{S}_{i, s}\right]_{\mathcal{B}_{\mathcal{S}}}$, and by applying the definition of $\mathcal{B}_{\mathcal{S}}$ also

$$
\left[\mathcal{F}_{q, i, s}\right]_{\mathcal{B}_{\mathcal{E}}}=\left\{\begin{array}{lll}
\ddot{\Phi}^{\langle 10\rangle}-\ddot{\Phi}^{\langle 2\rangle} & \text { if } \quad i=1, s=0, \\
\ddot{\Phi}^{\langle 2\rangle} & \text { if } \quad i=1, s=1, \\
\ddot{\Phi}^{\langle 10\rangle}-\ddot{\Phi}^{\langle 3,4\rangle} & \text { if } \quad i=2, s=0, \\
\ddot{\Phi}^{\langle s+2\rangle} & \text { if } \quad i=2, s=1,2, \\
\ddot{\Phi}^{\langle 10\rangle}-\ddot{\Phi}^{\langle 5,9\rangle} & \text { if } \quad i=3, s=0, \\
\ddot{\Phi}^{\langle s+4\rangle} & \text { if } \quad i=3, s=1, \ldots, 5,
\end{array}\right.
$$

where $\ddot{\Phi}=\ddot{\Phi}_{q, \infty}$. On the other hand, by the definition of $X_{q, i, s}$ we have the equality $\left[X_{q, i, s}\right]=\epsilon^{q}\left[\mathcal{F}_{q, i, s}\right]$ in $\mathrm{K}_{0}(\mathbb{X})$, where $\epsilon^{q}:=1$, if $q \geq 1$ (respectively, $\epsilon^{q}:=-1$, if $q \leq 0$ ); consequently,

(**)

$$
\operatorname{dim}_{k} X_{q, i, s}=\epsilon^{q} \cdot\left[\mathcal{F}_{q, i, s}\right]_{\mathcal{B}_{\mathcal{E}}} .
$$

Now the formula 2.3(**) from the assertion of the theorem follows immediately by $(*)$ and $(* *)$, since $\epsilon_{\gamma}=\epsilon^{q(\gamma)}, \Xi \gamma=\epsilon^{q(\gamma)} \ddot{\Phi}_{q(\gamma), \infty}, M_{i, s}(\gamma) \cong \hat{X}_{q(\gamma), i, s}$ and $\operatorname{dim}_{k} \hat{X}=$ $\xi\left(\operatorname{dim}_{k} X\right)$, for any $X$ in $\bmod (\Lambda)($ see $2.2(*), 3.12(* *)$; 4.6; 3.1; respectively). In this way the proof is complete.

\section{8}

The comparison theorem yields also a possibility to determine the mono-epi property (and eventually the mono-epi type) for indecomposable $\Lambda$-modules and $R$-modules being objects of $\tilde{\mathcal{S}}$. We start by formulating the basic definition.

Let $(Q, \Delta)$ be for a moment an arbitrary bounded quiver and $A=k Q / \Delta$ the associated $k$-algebra (respectively, $k$-category). As usually we identify right $A$-modules with representations of $(Q, \Delta)$. We denote by $\Omega=\Omega(Q, \Delta)$ the set of all oriented paths $\delta$ in $Q$ of positive length, which do not belong to $\Delta$. We say that $M=\left(M_{v}, M_{\gamma}\right)_{v \in Q_{0}, \gamma \in Q_{1}} \in \bmod (A)$ is a path mono-epi representation (mono-epi epresentation, in short), if for any $\delta=$ $\gamma_{1} \ldots \gamma_{n} \in \Omega$, where $n \geq 1$, the associated $k$-linear structure map $M_{\delta}:=M_{\gamma_{n}} \circ \ldots \circ M_{\gamma_{1}}$ : $M_{s(\delta)} \rightarrow M_{h(\delta)}$ is a monomorphism or an epimorphism. Clearly, if $M$ is as above and $\operatorname{dim}_{k} M=\left[d_{v}\right]_{v \in Q_{0}}$ then $M_{\delta}$ is a monomorphism if and only if $d_{s(\delta)} \leq d_{h(\delta)}$ (respectively, $\overline{M_{\delta}}$ is an epimorphism if and only if $d_{s(\delta)} \geq d_{h(\delta)}$ ), for any $\delta \in \Omega$. Moreover, for any pair $\delta, \delta^{\prime} \in \Omega$ with $s\left(\delta^{\prime}\right)=h(\delta)(=: v)$, the inequalities $d_{s(\delta)}, d_{h\left(\delta^{\prime}\right)} \geq d_{v}$ implies $d_{s(\delta)}=d_{v}$ or $d_{v}=d_{h\left(\delta^{\prime}\right)}$ (if $M_{\delta \delta^{\prime}}$ is a monomorphism or an epimorphism, respectively), in case $\delta \delta^{\prime} \in \Omega$; 
and $d_{s(\delta)}, d_{h\left(\delta^{\prime}\right)}, d_{v} \neq 0$ implies the inequality $d_{s(\delta)}+d_{h\left(\delta^{\prime}\right)} \leq d_{v}$, in case $\delta \delta^{\prime} \in \Delta\left(M_{\delta}\right.$ must be a monomorphism and $M_{\delta^{\prime}}$ an epimorphism with $\left.\operatorname{Im} M_{\delta} \subseteq \operatorname{Ker} M_{\delta^{\prime}}\right)$.

Note that if the direct sum $M:=M^{\prime} \oplus M^{\prime \prime}$ of $\operatorname{modules}$ in $\bmod (A)$ is a mono-epi representation then both, $M^{\prime}$ and $M^{\prime \prime}$ do so (the opposite implication is not true in general).

\section{9}

Returning to the setup from the beginning of Section 4, which precedes the formulation of Theorem 4.1, notice that the set $\Omega$ defined in Section 3.9 differs formally from $\Omega(Q, \Delta)$. Nevertheless, due to the commutativity relations in $\Delta$, to examine the mono-epi property for $M$ in $\bmod (\Lambda)$ it suffices to consider just the set $\Omega$, since for any pair of parallel oriented paths $\delta, \delta^{\prime}$ in $Q$ with $s\left(\delta^{\prime}\right)=s\left(\delta^{\prime}\right)=j$ and $h(\delta)=h\left(\delta^{\prime}\right)=i$, where $(i, j) \in \Omega$, we have the equality $M_{\delta}=M_{\delta^{\prime}}$.

Recall that $T$ is a tilting bundle, so as stated in Section 3.9, each $\theta_{j, i}: T_{i} \rightarrow T_{j}$, for $(i, j) \in \Omega$, is either a monomorphism (if $\operatorname{rk}\left(T_{i}\right) \leq \operatorname{rk}\left(T_{j}\right)$ ) or an epimorphism (if $\operatorname{rk}\left(T_{i}\right)>$ $\left.\operatorname{rk}\left(T_{j}\right)\right)$. We will show that for a suitable choice of $\mathcal{F} \in \operatorname{coh}_{\geq}(\mathbb{X})$ the module $M:=\Psi(\mathcal{F})=$ $\operatorname{Hom}_{\mathbb{X}}(T, \mathcal{F}) \in \bmod _{\geq}(\Lambda)$ is mono-epi.

More precisely, denote by $\Omega_{e}$ the set of all $(i, j) \in \Omega \operatorname{such}$ that $\operatorname{rk}\left(T_{i}\right)>\operatorname{rk}\left(T_{j}\right)$; i.e.

$$
\begin{aligned}
\Omega_{e}= & \{(4,5),(5,6),(6,7),(4,6),(4,7),(5,7),(3,6),(3,7),(2,7),(3,8), \\
& (4,9),(5,10),(8,9),(9,10),(8,10),(3,9),(4,10),(3,10),(2,10)\},
\end{aligned}
$$

and by $\Omega_{m}$ its completion $\Omega_{m}:=\Omega \backslash \Omega_{e}$ consisting of all $(i, j) \in \Omega$ such that $\operatorname{rk}\left(T_{i}\right) \leq$ $\operatorname{rk}\left(T_{j}\right)$. We also set

$$
\Omega_{m}^{1}:=\{(3,4),(1,6),(2,5),(1,9),(2,8)\}, \quad \Omega_{m}^{\infty}:=\{(2,6),(3,5),(1,10),(2,9)\}
$$

and $\Omega_{m}^{<1}:=\Omega_{m} \backslash\left(\Omega_{m}^{1} \cup \Omega_{m}^{\infty}\right)$.

Let $\mathcal{F}$ be a sheaf in $\operatorname{coh}_{+}(\mathbb{X})$. We set

$$
M_{(i, j)}:=\operatorname{Hom}_{\mathbb{X}}\left(\theta_{j, i}, \mathcal{F}\right): M_{j} \rightarrow M_{i}
$$

for any $(i, j) \in \Omega$, where $M_{l}:=\operatorname{Hom}_{\mathbb{X}}\left(T_{l}, \mathcal{F}\right)$ for all $l \in[10]$. Observe that the maps $M_{(i, j)}$, for $(i, j) \in \Omega$, are the all possible compositions of the maps $M_{\alpha}, \alpha \in Q_{1}$, which can be nonzero.

Proposition 4.10 Let $\mathcal{F}$ be an indecomposable sheaf in $\operatorname{coh}_{\geq}(\mathbb{X})$ such that $1 \leq \mu(\mathcal{F}) \leq$ $\infty$.

- $\quad$ The map $M_{(i, j)}$ is a monomorphism for every $(i, j) \in \Omega_{e}$ and an epimorphism for every $(i, j) \in \Omega_{m}^{<1}$, additionally it is a monomorphism for every $(i, j) \in \Omega_{m}^{\infty}$ and an epimorphism for every $(i, j) \in \Omega_{m}^{1}$, provided $1<\mu(\mathcal{F})<\infty$.

- If $\mu(\mathcal{F})=1$ then $M_{(i, j)}$ is a monomorphism for every $(i, j) \in \Omega_{e} \cup \Omega_{m}^{\infty}$ and an epimorphism for every $(i, j) \in \Omega_{m}^{<1}$; moreover, for each pair $(i, j) \in \Omega_{m}^{1}$, the map $M_{(i, j)}$ is an isomorphism for all $\mathcal{F}$ lying in any tube from the family $\mathcal{T}^{1}$ except of the one distinguished exceptional tube $\mathcal{T}_{\lambda(i, j)}^{1}, \lambda(i, j) \in\left\{\lambda_{1}, \lambda_{2}, \lambda_{3}\right\}$, and it is a monomorphism (respectively, an epimorphism) for all but precisely one quasi-simple objects $\mathcal{F}$ in $\mathcal{T}_{\lambda(i, j)}^{1}$, which one we will denote by $\mathcal{F}^{\prime}$ (respectively, $\mathcal{F}^{\prime \prime}$ ).

- If $\mu(\mathcal{F})=\infty$ then $M_{(i, j)}$ is a monomorphism for every $(i, j) \in \Omega_{e}$ and an epimorphism for every $(i, j) \in \Omega_{m}^{<1} \cup \Omega_{m}^{1}$; moreover, for each pair $(i, j) \in \Omega_{m}^{\infty}$, the map 
$M_{(i, j)}$ is an isomorphism for all $\mathcal{F}$ lying in any tube from the family $\mathcal{T}^{\infty}$ except of the one distinguished exceptional tube $\mathcal{T}_{\lambda(i, j)}^{\infty}, \lambda(i, j) \in\left\{\lambda_{1}, \lambda_{2}, \lambda_{3}\right\}$, and it is a monomorphism (respectively, an epimorphism) for all but precisely one quasi-simple objects $\mathcal{F}$ in $\mathcal{T}_{\lambda(i, j)}^{\infty}$, which one we will denote by $\mathcal{F}^{\prime}$ (respectively, $\mathcal{F}^{\prime \prime}$ ).

Proof Fix an indecomposable $\mathcal{F}$ in $\operatorname{coh}_{\geq}(\mathbb{X})$. Clearly, $M_{(i, j)}$ is always a monomorphism, if $\operatorname{rk}\left(T_{i}\right)>\operatorname{rk}\left(T_{j}\right)$. Now assume that $\operatorname{rk}\left(T_{i}\right) \leq \operatorname{rk}\left(T_{j}\right)$, equivalently, $\theta_{j, i}$ is a monomorphisms. We have a short exact sequence

$$
0 \rightarrow T_{i} \stackrel{\theta_{j, i}}{\longrightarrow} T_{j} \rightarrow Q_{i, j} \rightarrow 0
$$

where $Q_{i, j}:=\operatorname{Coker} \theta_{j, i}$. The cokernel $Q_{i, j}$ is an exceptional sheaf as a result of a mutation of the exceptional pair $\left(T_{i}, T_{j}\right)$ (see $[17,4.2]$ ), so it is indecomposable. Hence, $Q_{i, j}$ lies in exceptional tube in $\operatorname{coh}(\mathbb{X})$ with slope

$$
\mu\left(Q_{i, j}\right)=\frac{\operatorname{deg}\left(T_{j}\right)-\operatorname{deg}\left(T_{i}\right)}{\operatorname{rk}\left(T_{j}\right)-\operatorname{rk}\left(T_{i}\right)} .
$$

(The slopes of all $Q_{i, j}$, for $(i, j) \in \Omega_{m}$, can be easily computed by (**) and Theorem 3.6(a), see Table 1 below). Moreover, each $Q_{i, j}$ belongs to $\operatorname{coh}_{\geq}(\mathbb{X})$, since the functor $\operatorname{Ext}_{\mathbb{X}}^{1}(T,-)$ is right exact.

Consider the long exact sequence for the functor $\operatorname{Hom}_{\mathbb{X}}(-, \mathcal{F})$, induced by $(*)$. Due to the equality $\operatorname{Ext}_{\mathbb{X}}^{1}(T, \mathcal{F})=0$ its beginning looks as follows:

$$
0 \rightarrow \operatorname{Hom}_{\mathbb{X}}\left(Q_{i, j}, \mathcal{F}\right) \rightarrow \operatorname{Hom}_{\mathbb{X}}\left(T_{j}, \mathcal{F}\right) \rightarrow \operatorname{Hom}_{\mathbb{X}}\left(T_{i}, \mathcal{F}\right) \rightarrow \operatorname{Ext}_{\mathbb{X}}^{1}\left(Q_{i, j}, \mathcal{F}\right) \rightarrow 0 .
$$

Consequently, $M_{(i, j)}$ is a monomorphism if and only if $\operatorname{Hom}_{\mathbb{X}}\left(Q_{i, j}, \mathcal{F}\right)=0$. Notice that this is always the case if $\mu(\mathcal{F})<\mu\left(Q_{i, j}\right)$, or if $\mu(\mathcal{F})=\mu\left(Q_{i, j}\right)$ and $\mathcal{F}$ belongs to another tube than $Q_{i, j}$. Moreover, in the tube of $\operatorname{coh}(\mathbb{X})$ containing $Q_{i, j}$ there exists precisely one quasi-simple object $\mathcal{F}^{\prime}=\mathcal{F}^{\prime}(i, j)$ with $\operatorname{Hom}_{\mathbb{X}}\left(Q_{i, j}, \mathcal{F}^{\prime}\right) \neq 0$; namely, the quasi-top of $Q_{i, j}$. Note that it belongs to $\mathrm{coh}_{\geq}(\mathbb{X})$, since all epimorphic images of $Q_{i, j}$ have this property.

Similarly, $M_{(i, j)}$ is an epimorphism if and only if $\operatorname{Ext}_{\mathbb{X}}^{1}\left(Q_{i, j}, \mathcal{F}\right)=0$, or equivalently due to Serre duality if $\operatorname{Hom}_{\mathbb{X}}\left(\mathcal{F}, \tau_{\mathbb{X}}\left(Q_{i, j}\right)\right)=0$. In particular, this is always the case if $\mu(\mathcal{F})>\mu\left(Q_{i, j}\right)$, or if $\mu(\mathcal{F})=\mu\left(Q_{i, j}\right)$ and $\mathcal{F}$ belongs to another tube than $Q_{i, j}$. Moreover, in the tube of $\operatorname{coh}(\mathbb{X})$ containing $Q_{i, j}$ there exists precisely one quasi-simple object $\mathcal{F}^{\prime \prime}=\mathcal{F}^{\prime \prime}(i, j)$ with $\operatorname{Hom}_{\mathbb{X}}\left(\mathcal{F}^{\prime \prime}, \tau_{\mathbb{X}}\left(Q_{i, j}\right)\right) \neq 0$; namely, the quasi-socle of $\tau_{\mathbb{X}}\left(Q_{i, j}\right)$. We claim that if $\mu\left(Q_{i, j}\right) \geq 1$ then $\mathcal{F}^{\prime \prime}$ belongs to $\operatorname{coh}_{+}(\mathbb{X})$. Notice that eventual problems can appear only for the pairs $(i, j) \in \Omega_{m}$ with $\mu\left(Q_{i, j}\right)=1$, for those additionally $\rho\left(Q_{i, j}\right)=6$. Nevertheless our claim holds true also in this case, since by the shape of the dimension vectors of the $\Lambda$-modules $S$ from the mouths of the tubes $\mathcal{T}_{\lambda_{3}}^{1}$ and $\mathcal{T}_{\lambda_{2}}^{1}$ (can be immediately determined by the formulas (*) and (**) in Section 4.7) one easily indicates for each of the five pairs $(i, j) \in \Omega_{m}^{1}$ the (unique) module $S=S^{\prime \prime}(i, j)$ such that the structure map

Table 1 Data for the cokernels of maps between summands of $T$

\begin{tabular}{lllllllllllllllllll}
\hline$(i, j)$ & $(1,2)$ & $(2,3)$ & $(3,4)$ & $(1,3)$ & $(2,4)$ & $(1,4)$ & $(1,5)$ & $(1,6)$ & $(2,5)$ & $(2,6)$ & $(3,5)$ & $(1,8)$ & $(1,9)$ & $(1,10)$ & $(2,8)$ & $(2,9)$ \\
\hline $\operatorname{deg}\left(Q_{i, j}\right)$ & 1 & 1 & 1 & 2 & 2 & 3 & 3 & 2 & 2 & 1 & 1 & 2 & 2 & 1 & 1 & 1 \\
$\operatorname{rk}\left(Q_{i, j}\right)$ & 2 & 2 & 1 & 4 & 3 & 5 & 4 & 2 & 2 & 0 & 0 & 3 & 2 & 0 & 1 & 0 \\
$\mu\left(Q_{i, j}\right)$ & $\frac{1}{2}$ & $\frac{1}{2}$ & 1 & $\frac{1}{2}$ & $\frac{2}{3}$ & $\frac{3}{5}$ & $\frac{3}{4}$ & 1 & 1 & $\infty$ & $\infty$ & $\frac{2}{3}$ & 1 & $\infty$ & 1 & $\infty$ \\
\hline
\end{tabular}


$S_{(i, j)}: S_{j} \rightarrow S_{i}$ in $S$ is not an epimorphism (see $L^{1}$ from Section 6 and Table 1, cf. also Remark 4.11).

Now, the assertions follow from the considerations above by a simple analysis of Table 1 containing the slopes of all the cokernels $Q_{i, j}$, for $(i, j) \in \Omega_{m}$.

Remark 4.11 (a) For every $(i, j) \in \Omega_{m}^{1}$ (respectively, $\left.(i, j) \in \Omega_{m}^{\infty}\right)$ we have $\operatorname{deg}\left(Q_{i, j}\right) \in$ $\{1,2\}$ (respectively, $\operatorname{deg}\left(Q_{i, j}\right)=1$ ), so due to the formula 3.4(**) either $\rho\left(Q_{i, j}\right)=6$ and $\ell\left(Q_{i, j}\right) \leq 2$ or $\rho\left(Q_{i, j}\right)=3$ and $\ell\left(Q_{i, j}\right)=1$ (respectively, $\rho\left(Q_{i, j}\right)=6$ and $\ell\left(Q_{i, j}\right)=1$ ). Moreover, $\ell\left(Q_{i, j}\right)=1$ if and only if $\mathcal{F}^{\prime \prime}=\tau_{\mathbb{X}}\left(\mathcal{F}^{\prime}\right)$ and $\ell\left(Q_{i, j}\right)=2$ if and only if $\mathcal{F}^{\prime \prime}=$ $\tau_{\mathbb{X}}^{2}\left(\mathcal{F}^{\prime}\right)$. In particular, we have always $\mathcal{F}^{\prime} \neq \mathcal{F}^{\prime \prime}$ and the positions of the pair $\left(\mathcal{F}^{\prime}, \mathcal{F}^{\prime \prime}\right)$ allow to recover precisely the position of $Q_{i, j}$ in the tube.

(b) Using the list $L^{1}$, one can also easily indicate for each of the five pairs $(i, j) \in \Omega_{m}^{1}$ (the unique) $S$ from the union of the mouths of the tubes $\mathcal{T}_{\lambda_{3}}^{1}$ and $\mathcal{T}_{\lambda_{2}}^{1}$, such that the structure map $S_{(i, j)}: S_{j} \rightarrow S_{i}$ in $S$ is not a monomorphism. The same can be done for each of the four pairs $(i, j) \in \Omega_{m}^{\infty}$, in an epimorphism as well as a monomorphism version for the map $S_{(i, j)}$, where now $S$ belongs to the mouth of the tube $\mathcal{T}_{\lambda_{3}}^{\infty}$ (use $L^{\infty}$ from Section 6).

(c) For any $(i, j) \in \Omega_{m}^{1}$ we have $\lambda(i, j)=\lambda_{2}$ or $\lambda(i, j)=\lambda_{3}$ (i.e. $\rho\left(Q_{i, j}\right)=3$ or $\rho\left(Q_{i, j}\right)=6$, respectively). The sequence 6,6,3,3,6 describes the ranks of the tubes $\mathcal{T}_{\lambda(i, j)}^{1}$ (containing $Q_{i, j}$ ) for the consecutive five pairs $(i, j) \in \Omega_{m}^{1}$, ordered as in the definition of the set $\Omega_{m}^{1}$ (apply (b)). For any $(i, j) \in \Omega_{m}^{\infty}$ we have $\lambda(i, j)=\lambda_{3}$, since $\rho\left(Q_{i, j}\right)=6$ (see (a)).

Now we consider the analogous problem for modules $M:=\Psi(\mathcal{F}[1])=\operatorname{Ext}_{\mathbb{X}}^{1}(T, \mathcal{F}) \in$ $\bmod _{-}(\Lambda)$, where $\mathcal{F} \in$ coh_$_{-}(\mathbb{X})$.

We set

$$
\Omega_{e}^{0}:=\{(4,5),(3,6),(2,7),(3,8),(8,9),(3,9),(2,10)\}
$$

and $\Omega_{e}^{>0}:=\Omega_{e} \backslash \Omega_{e}^{0}$. Let $\mathcal{F}$ be as above then we set

$$
M_{(i, j)}:=\operatorname{Ext}_{\mathbb{X}}^{1}\left(\theta_{j, i}, \mathcal{F}\right): M_{j} \rightarrow M_{i}
$$

for any $(i, j) \in \Omega$, where $M_{l}:=\operatorname{Ext}_{\mathbb{X}}^{1}\left(T_{l}, \mathcal{F}\right)$ for all $l \in[10]$.

Proposition 4.12 Let $\mathcal{F}$ be an indecomposable sheaf in coh_( $\mathbb{X})$ such that $\mu(\mathcal{F}) \leq 0$.

- The map $M_{(i, j)}$ is an epimorphism for every $(i, j) \in \Omega_{m}$ and a monomorphisms for every $(i, j) \in \Omega_{e}^{>0}$, additionally it is a monomorphism for every $(i, j) \in \Omega_{e}^{0}$, provided $\mu(\mathcal{F})<0$.

- If $\mu(\mathcal{F})=0$ then $M_{(i, j)}$ is an epimorphism for every $(i, j) \in \Omega_{m}$ and a monomorphisms for every $(i, j) \in \Omega_{e}^{>0}$; moreover, for each pair $(i, j) \in \Omega_{e}^{0}$, the map $M_{(i, j)}$ is an isomorphism for all $\mathcal{F}$ lying in any tube from the family $\mathcal{T}^{0}$ except of the one distinguished exceptional tube $\mathcal{T}_{\lambda(i, j)}^{0}, \lambda(i, j) \in\left\{\lambda_{1}, \lambda_{2}, \lambda_{3}\right\}$, and it is a monomorphism (respectively, an epimorphism) for all but precisely one quasi-simple objects $\mathcal{F}$ in $\mathcal{T}_{\lambda(i, j)}^{0}$, which one we will denote by $\mathcal{F}^{\prime}$ (respectively, $\mathcal{F}^{\prime \prime}$ ).

Proof We use arguments similar to those from the proof of Proposition 4.10. Fix an indecomposable $\mathcal{F}$ in coh_( $\mathbb{X})$. The category $\operatorname{coh}(\mathbb{X})$ is hereditary so the functor $\operatorname{Ext}_{\mathbb{X}}^{1}(-, \mathcal{F})$ is right exact. Consequently, $M_{(i, j)}$ is an epimorphism for every $(i, j) \in \Omega_{m}$, since in this case $\theta_{j, i}: T_{i} \rightarrow T_{j}$ is a monomorphism. 
Now assume that $(i, j) \in \Omega_{e}$, equivalently, that $\theta_{j, i}$ is an epimorphism. Then we have a short exact sequence

$$
0 \rightarrow K_{i, j} \rightarrow T_{i} \stackrel{\theta_{j, i}}{\rightarrow} T_{j} \rightarrow 0
$$

where $K_{i, j}:=\operatorname{Ker} \theta_{j, i}$. The kernel $K_{i, j}$ is an exceptional sheaf as a result of a mutation of the exceptional pair $\left(T_{i}, T_{j}\right)$ (see $[17,4.2]$ ). Hence, $K_{i, j}$ belongs to an exceptional tube in $\operatorname{coh}(\mathbb{X})$ with slope

$$
\mu\left(K_{i, j}\right)=\frac{\operatorname{deg}\left(T_{i}\right)-\operatorname{deg}\left(T_{j}\right)}{\operatorname{rk}\left(T_{i}\right)-\operatorname{rk}\left(T_{j}\right)} .
$$

(The slopes of all $K_{i, j}$, for $(i, j) \in \Omega_{e}$, can be easily computed by (**) and Theorem 3.6(a), see Table 2 below).

Consider the long exact sequence for the functor $\operatorname{Hom}_{\mathbb{X}}(-, \mathcal{F})$, induced by $(*)$. Due to the equality $\operatorname{Hom}_{\mathbb{X}}(T, \mathcal{F})=0$ its nontrivial part looks as follows:

$$
0 \rightarrow \operatorname{Hom}_{\mathbb{X}}\left(K_{i, j}, \mathcal{F}\right) \rightarrow \operatorname{Ext}_{\mathbb{X}}^{1}\left(T_{j}, \mathcal{F}\right) \rightarrow \operatorname{Ext}_{\mathbb{X}}^{1}\left(T_{i}, \mathcal{F}\right) \rightarrow \operatorname{Ext}_{\mathbb{X}}^{1}\left(K_{i, j}, \mathcal{F}\right) \rightarrow 0 .
$$

Consequently, $M_{(i, j)}$ is an epimorphism if and only if $\operatorname{Ext}_{\mathbb{X}}^{1}\left(K_{i, j}, \mathcal{F}\right)=0$, or equivalently due to Serre duality if $\operatorname{Hom}_{\mathbb{X}}\left(\mathcal{F}, \tau_{\mathbb{X}}\left(K_{i, j}\right)\right)=0$. Notice that this is always the case if $\mu(\mathcal{F})>\mu\left(K_{i, j}\right)$, or if $\mu(\mathcal{F})=\mu\left(K_{i, j}\right)$ and $\mathcal{F}$ belongs to another tube than $K_{i, j}$. Moreover, in the tube in $\operatorname{coh}(\mathbb{X})$ containing $K_{i, j}$ there exists precisely one quasi-simple object $\mathcal{F}^{\prime \prime}=\mathcal{F}^{\prime \prime}(i, j)$ with $\operatorname{Hom}_{\mathbb{X}}\left(\mathcal{F}^{\prime \prime}, \tau_{\mathbb{X}}\left(K_{i, j}\right)\right) \neq 0$; namely, the quasi-socle of $\tau_{\mathbb{X}}\left(K_{i, j}\right)$.

Similarly, $M_{(i, j)}$ is a monomorphism if and only if $\operatorname{Hom}_{\mathbb{X}}\left(K_{i, j}, \mathcal{F}\right)=0$. This is always the case if $\mu(\mathcal{F})<\mu\left(K_{i, j}\right)$, or if $\mu(\mathcal{F})=\mu\left(K_{i, j}\right)$ and $\mathcal{F}$ belongs to another tube than $K_{i, j}$. Moreover, in the tube in $\operatorname{coh}(\mathbb{X})$ containing $K_{i, j}$ there exists precisely one quasi-simple object $\mathcal{F}^{\prime \prime}=\mathcal{F}^{\prime \prime}(i, j)$ with $\operatorname{Hom}_{\mathbb{X}}\left(K_{i, j}, \mathcal{F}^{\prime \prime}\right) \neq 0$; namely, the quasi-top of $K_{i, j}$.

We claim that if $\mu\left(K_{i, j}\right) \leq 0$ (in fact, $\mu\left(K_{i, j}\right)=0$, see Table 2) then both, $\mathcal{F}^{\prime \prime}$ and $\mathcal{F}^{\prime}$, belong to coh_( $\mathbb{X})$. Notice that eventual problems can appear only for the pairs $(i, j) \in \Omega_{e}$ with $\mu\left(K_{i, j}\right)=0$, for those additionally $\rho\left(K_{i, j}\right)=6$. Nevertheless, our claim is valid also in this case, since by the shape of the dimension vectors of the $\Lambda$-modules $S$ from the mouths of the tubes $\mathcal{T}_{\lambda_{3}}^{0}$ and $\mathcal{T}_{\lambda_{2}}^{0}$ (can be determined by the formulas (*) and (**) in Section 4.7, for each of the seven pairs $(i, j) \in \Omega_{e}^{0}$, one can easily indicate among the modules $S$ the (unique) pair, $S=S^{\prime \prime}(i, j)$ and $S=S^{\prime}(i, j)$, such that the structure map $S_{(i, j)}: S_{j} \rightarrow S_{i}$ in $S$ is not an epimorphism, for $S=S^{\prime \prime}(i, j)$, respectively is not a monomorphism, for $S=S^{\prime}(i, j)$ (see $L^{0}$ from Section 6 and Table 2, cf. also Remark 4.13).

Now the assertions follow from the considerations above by a simple analysis of Table 2 containing the slopes of all the kernels $K_{i, j}$, for $(i, j) \in \Omega_{e}$.

Remark 4.13 (a) For every $(i, j) \in \Omega_{e}^{0}$ we have now $\operatorname{rk}\left(K_{i, j}\right) \in\{1,2\}$, so due to $3.4(* *)$ either $\rho\left(K_{i, j}\right)=6$ and $\ell\left(K_{i, j}\right) \leq 2$ or $\rho\left(K_{i, j}\right)=3$ and $\ell\left(K_{i, j}\right)=1$. Moreover, $\ell\left(K_{i, j}\right)=1$ if and only if $\mathcal{F}^{\prime \prime}=\tau_{\mathbb{X}}\left(\mathcal{F}^{\prime}\right)$, and $\ell\left(K_{i, j}\right)=2$ if and only if $\mathcal{F}^{\prime \prime}=\tau_{\mathbb{X}}^{2}\left(\mathcal{F}^{\prime}\right)$. In particular, we

Table 2 Data for the kernels of maps between summands of $T$

$(\mathrm{i}, \mathrm{j}) \quad(4,5)(5,6)(6,7)(4,6)(4,7)(5,7)(3,6)(3,7)(2,7)(3,8)(4,9)(5,10)(8,9)(9,10)(8,10)(3,9)(4,10)(3,10)(2,10)$

\begin{tabular}{llllllllllllllllllll}
\hline $\operatorname{deg}\left(K_{i, j}\right)$ & 0 & 1 & 1 & 1 & 2 & 2 & 0 & 1 & 0 & 0 & 1 & 2 & 0 & 1 & 1 & 0 & 2 & 1 & 0 \\
$\operatorname{rk}\left(K_{i, j}\right)$ & 1 & 2 & 2 & 3 & 5 & 4 & 2 & 4 & 2 & 1 & 3 & 4 & 1 & 2 & 3 & 2 & 5 & 4 & 2 \\
$\mu\left(K_{i, j}\right)$ & 0 & $\frac{1}{2}$ & $\frac{1}{2}$ & $\frac{1}{3}$ & $\frac{2}{5}$ & $\frac{1}{2}$ & 0 & $\frac{1}{4}$ & 0 & 0 & $\frac{1}{3}$ & $\frac{1}{2}$ & 0 & $\frac{1}{2}$ & $\frac{1}{3}$ & 0 & $\frac{2}{5}$ & $\frac{1}{4}$ & 0 \\
\hline
\end{tabular}


have always $\mathcal{F}^{\prime} \neq \mathcal{F}^{\prime \prime}$ and the positions of the pair $\left(\mathcal{F}^{\prime}, \mathcal{F}^{\prime \prime}\right)$ allow to recover precisely the position of $K_{i, j}$ in the tube.

(b) For any $(i, j) \in \Omega_{e}^{0}$ we have $\lambda(i, j)=\lambda_{2}$ or $\lambda(i, j)=\lambda_{3}$ (i.e. $\rho\left(K_{i, j}\right)=3$ or $\rho\left(K_{i, j}\right)=6$, respectively). The sequence $3,3,3,3,6,6,6$ describes the ranks of the tubes $\mathcal{T}_{\lambda(i, j)}^{0}$ (containing $K_{i, j}$ ) for the consecutive seven pairs $(i, j) \in \Omega_{e}^{0}$, ordered as in the definition of the set $\Omega_{e}^{0}$.

(c) For $(i, j) \in \Omega_{e}^{0}$, the kernel $K_{i, j}$ belongs to coh_( $\left.\mathbb{X}\right)$ if and only if $(i, j) \neq(2,7)$, since then the equality $\operatorname{Hom}_{\mathbb{X}}\left(T, K_{i, j}\right)=0$ is equivalent to $\operatorname{Hom}_{\mathbb{X}}\left(T_{1}, K_{i, j}\right)=0$.

Corollary 4.14 Let $M$ be an indecomposable $\Lambda$-module and $\underline{\operatorname{dim}_{k}} M=\left[d_{l}\right]_{l \in[10]}$.

(a) If $\mu(M) \in \overline{\mathbb{Q}}^{\prime}$ and $M$ does not belong to the rank-6 tube in case $\mu(M)=\infty$ (respectively, to the union of the two biggest tubes, in case $\mu(M)=0,1$ ) then $M$ is a mono-epi representation with $M_{(3,8)}, M_{(4,9)}, M_{(5,10)}$ being monomorphisms. In particular,

(i) if $\mu(M) \in(1, \infty)$ then

$$
d_{1}+d_{7} \leq d_{6} \leq d_{2} \leq d_{5} \leq d_{3} \leq d_{4} \quad \text { and } \quad d_{10} \leq d_{1} \leq d_{9} \leq d_{2} \leq d_{8},
$$

(ii) if $\mu(M) \in(-\infty, 0)$ then

$$
d_{1}+d_{7} \leq d_{2} \leq d_{6} \leq d_{3} \leq d_{5} \leq d_{4} \quad \text { and } \quad d_{1} \leq d_{10} \leq d_{2} \leq d_{9} \leq d_{8},
$$

(iii) if $\mu(M)=\infty$ then $d_{2}=d_{6}, d_{3}=d_{5} ; d_{1}=d_{10}, d_{2}=d_{9}$ and $\operatorname{dim}_{k} M$ satisfies simultaneously the inequalities (i) and (ii),

(iv) if $\mu(M)=1$ then $d_{1}=d_{6}, d_{3}=d_{4}, d_{2}=d_{5} ; d_{1}=d_{9}, d_{2}=d_{8}$ and $\operatorname{dim}_{k} M$ satisfies the inequalities $(i)$,

(v) if $\mu(M)=0$ then $d_{2}=d_{7}, d_{3}=d_{6}, d_{4}=d_{5} ; d_{2}=d_{10}, d_{8}=d_{9}$ and $\operatorname{dim}_{k} M$ satisfies the inequalities (ii).

(b) If $\mu(M)=\infty$ and $M$ is a quasi simple module from the rank-6 tube then $M$ is a mono-epi representation with $M_{(3,8)}, M_{(4,9)}, M_{(5,10)}$ being monomorphisms

(c) If $\mu(M)=1$ and $M$ is a quasi-simple from one of the two biggest tubes then $M$ is a mono-epi representation with $M_{(3,8)}, M_{(4,9)}, M_{(5,10)}$ being monomorphisms

(d) If $\mu(M)=0$ and $M$ is a quasi-simple from one of the two biggest tubes then $M$ is a mono-epi representation, with $M_{(4,9)}, M_{(5,10)}$ being always monomorphisms, and $M_{(3,8)}$ being a monomorphism except for precisely one distinguished quasi-simple module $M=M^{\prime}$ in the biggest tube, for whom $M_{(3,8)}$ is an epimorphism.

Proof An immediate consequence of Propositions 4.10, 4.12 and Remarks 4.11, 4.13.

Remark 4.15 Applying this method one can also examine the remaining indecomposable $\Lambda$-modules $M$ with respect to the property of being mono-epi representation (i.e. the preprojective and preinjective ones).

\section{Interval Decompositions and Mono-epi Types}

\section{1}

Now we introduce the notion of a so-called mono-epi type which allows to understand in a more systematic and deeper way the class of mono-epi representations. In particular, 
we show that in the case of the category $\tilde{A}(6)$ the mono-epi types determine the interval decomposition of mono-epi representations which belong to these types. We apply a precise formula for their multiplicity vectors to prove Theorem 2.8 .

Let again, as in Section $4.8,(Q, \Delta)$ be for a moment an arbitrary bounded quiver and $A=k Q / \Delta$ the associated $k$-algebra (respectively, $k$-category). We fix the two element set $\{e, m\}$ of symbols. A map $t: \Omega \rightarrow\{e, m\}$, where $\Omega=\Omega(Q, \Delta)$ is as in Section 4.8 will be called a sincere abstract mono-epi type for the algebra $A$ (an abstract mono-epi type, in short), if it satisfies the following natural condition: for any pair $\delta, \delta^{\prime} \in \Omega$, with $h(\delta)=s\left(\delta^{\prime}\right)$ and $t(\delta)=t\left(\delta^{\prime}\right)$, we have $\delta \delta^{\prime} \in \Omega$ and $t\left(\delta \delta^{\prime}\right)=t(\delta)\left(=t\left(\delta^{\prime}\right)\right)$. Additionally, we say that $t$ as above is strict, if for any pair $\delta, \delta^{\prime} \in \Omega$, with $h(\delta)=s\left(\delta^{\prime}\right)$ and $\delta \delta^{\prime} \in \Omega$, the equality $t(\delta)=e$ always implies $t\left(\delta^{\prime}\right)=e$ (equivalently, $t\left(\delta^{\prime}\right)=m$ implies $t(\delta)=m$ ). If $Q$ is acyclic and $t(\delta)=t\left(\delta^{\prime}\right)$ for each pair of parallel paths $\delta, \delta^{\prime} \in \Omega$ (i.e. such that $s(\delta)=s\left(\delta^{\prime}\right)$ and $\left.h(\delta)=h\left(\delta^{\prime}\right)\right)$, then to any abstract mono-epi type $t$ we associate the relation $\prec=\prec_{t} \subseteq\left(Q_{0}\right)^{2}$ consisting of all the pairs $(s(\delta), h(\delta))$, for $\delta \in \Omega$ with $t(\delta)=m$, and $(h(\delta), s(\delta))$, for $\delta \in \Omega$ with $t(\delta)=e$. It occurs that in good situations the reflexive closure $\preceq=\preceq_{t}$ of the relation $\prec$ yields an ordering of the vertex set $Q_{0}$.

We say that the (mono-epi) representation $M$ in $\bmod (A)$ (respectively, the vector $d=$ $\left.\left[d_{v}\right]_{v \in Q_{0}} \in \mathbb{N}^{Q_{0}}\right)$ is of type $t$, or equivalently belongs to $t$, if for any $\delta \in \Omega$, the map $M_{\delta}$ is an epimorphism (respectively, $d_{s(\delta)} \geq d_{h(\delta)}$ ), provided $t(\delta)=e$, and it is a monomorphism (respectively, $d_{s(\delta)} \leq d_{h(\delta)}$ ), provided $t(\delta)=m$. Clearly, $M$ belongs to $t$ if and only if so does $\operatorname{dim}_{k} M=\left[d_{v}\right]_{v \in Q_{0}}$. Notice that in case $M_{\delta}$ is an isomorphism for some $\delta \in \Omega$, such $t$ (if it exists) is not necessarily uniquely determined by $M$. If $d_{s(\delta)} \neq d_{h(\delta)}$ for all $\delta \in \Omega$, then $t$ must be strict and it is already uniquely determined for $M$. Observe also that the direct sum $M:=M^{\prime} \oplus M^{\prime \prime}$ of modules in $\bmod (A)$ belongs to the abstract mono-epi type $t$ if and only if both, $M^{\prime}$ and $M^{\prime \prime}$, do so.

Finally, an abstract mono-epi type $t$ is called a (real) mono-epi type, if there exists a sincere $M$ in $\bmod (A)$, which belongs to $t$. Observe that if $Q^{\prime}$ is a full subquiver of $Q, \Delta^{\prime}$ a restriction of the ideal $\Delta$ to $k Q^{\prime}$ (in the sense of path categories) and $A^{\prime}:=k Q^{\prime} / \Delta^{\prime}$ then the restriction $t^{\prime}:=t_{\mid}: \Omega\left(Q^{\prime}, \Delta^{\prime}\right) \rightarrow\{e, m\}$ of mono-epi type $t$ for $A$ is always a mono-epi type for $A^{\prime}$, called the restricted type.

\section{2}

For any $n \in \mathbb{N}_{1}$, we denote by $Q^{(n)}$ the linear quiver

$$
\stackrel{\alpha_{1}}{\longleftarrow} \stackrel{\circ}{\stackrel{\alpha_{2}}{\longleftarrow}} \stackrel{\circ}{\longleftarrow} \ldots \ldots \ldots \ldots
$$

and for any $i, j \in[n]$ with $i<j$, by $\delta_{j, i}$ the path $\alpha_{j-1} \ldots \alpha_{i}$ (by $\delta_{i, i}$ we mean the respective empty path). Moreover, we set $A^{(n)}:=k Q^{(n)}$ and $\bar{A}^{(n)}=k Q^{(n)} / \Delta^{(n)}$, where $\Delta^{(n)}$ is the two-sided ideal $\left\langle\delta_{n-1,1}\right\rangle$ in the path algebra $k Q^{(n)}$, which is admissible, if $n \geq 3$.

Recall that any path $\delta_{j, i}$ determines an indecomposable representation $I:=I_{[i, j]}$, called an interval, which is given by setting $I_{l}:=k$, if $i \leq l \leq j, I_{\alpha_{l}}:=\mathrm{id}_{k}$, if $i \leq l<j$, and $I_{l}:=0$ for all $l$ out of $\delta_{j, i}$. The set $\mathbb{S}=\mathbb{S}_{A^{(n)}}:=\left\{I_{[i, j]}: 1 \leq i \leq j \leq n\right\}$ yields a list of all, up to isomorphism, indecomposable nonisomorphic objects in $\bmod \left(A^{(n)}\right)$. For any $M$ in $\bmod \left(A^{(n)}\right)$, we have the so called interval decomposition $M \cong \bigoplus_{i \in \mathbb{S}} I^{a_{I}}$, for some uniquely determined multiplicities $a_{I} \in \mathbb{N}$. It is clear that for the algebra $\bar{A}^{(n)}$ an analogous role is played by the set $\overline{\mathbb{S}}=\mathbb{S}_{\bar{A}^{(n)}}:=\left\{I_{[i, j]}: 1 \leq i \leq j \leq n,(i, j) \neq(1, n)\right\}$; in particular, for any $M$ in $\bmod \left(\bar{A}^{(n)}\right)$ we have the interval decomposition $M \cong \bigoplus_{i \in \overline{\mathbb{S}}} I^{a_{I}}$. 
Now we discuss the problem "mono-epi representations versus mono-epi types" for these two kinds of algebras.

Lemma 5.3 Let $A=A^{(n)}$ and $Q=Q^{(n)}$, where $n \geq 2$.

(a) The mapping $t \mapsto \preceq_{t}$ yields a bijection between the sets of all abstract mono-epi types $t: \Omega \rightarrow\{e, m\}$ for the algebra $A$, where $\Omega=\Omega(Q, 0)$, and all linear orderings $\preceq$ of the set $Q_{0}=[n]$, i.e. permutations of $[n]$. In particular, via the bijection above the strict types $t$ correspond precisely to the linear orderings such that $1 \prec 2 \prec \ldots \prec p$ and $n \prec n-1 \prec \ldots \prec p$, for some $p \in[n]$.

(b) For each abstract mono-epi type t there exists a unique canonical vector $d(t)=\left[d_{i}\right] \in$ $\mathbb{N}^{Q_{0}}$ of type $t$, such that $d(t) \in[n]^{Q_{0}}$ and $d_{i}<d_{j}$, for all $i \prec j$. Moreover, if additionally $t$ is strict then for every $d=\left[d_{i}\right] \in \mathbb{N} Q_{0}$, which belongs to $t$, there exists precisely one, up to isomorphism, mono-epi representation $M=M(d)$ in $\bmod (A)$ with $\operatorname{dim}_{k} M=d$.

(c) Each mono-epi representation $M$ in $\bmod (A)$ belongs to some strict mono-epi type $t$.

Proof For $n=2$ all the assertions are trivially satisfied, so we assume that $n \geq 3$.

(a) First we briefly show that $\preceq=\preceq_{t}$ yields an ordering of [ $\left.n\right]$. (Note that now $\Omega=$ $\left.\left\{\delta_{j, i}: i<j\right\}\right)$. Antisymmetry of $\preceq$ holds, since $i \prec j$ means that $t\left(\delta_{j, i}\right)=e$, if $i<j$, and $t\left(\delta_{i, j}\right)=m$, if $i>j$; hence we have $j \nprec i$. To check transitivity of $\preceq$ fix $i, j, l \in[n]$ such that $i \prec j$ and $j \prec l$. Then there are six possible different $<$-orderings of the set $\{i, j, l\}$. If $i<j<l$ then $t\left(\delta_{l, j}\right)=t\left(\delta_{j, i}\right)=e$, so $t\left(\delta_{l, i}\right)=e$. In the case $i, l<j$ we have $t\left(\delta_{j, i}\right)=e$ and $t\left(\delta_{j, l}\right)=m$, and additionally $\delta_{j, i}=\delta_{j, l} \delta_{l, i}$, so $t\left(\delta_{l, i}\right)=e$, if $l>i$ (respectively, $\delta_{j, l}=\delta_{j, i} \delta_{i, l}$, so $t\left(\delta_{i, l}\right)=m$, if $i>l$ ); hence $i \prec l$. The remaining three cases are dual to the above onces. Notice that $\preceq$ is linear, since any two different vertices of $Q$ are connected by an oriented path (lying out of the ideal (0)). To finish the proof of the first part of (a), we associate to any linear ordering $\preceq$ of $[n]$ the map $t=t_{\preceq}: \Omega \rightarrow\{e, m\}$, given by setting $t\left(\delta_{j, i}\right)=e$, if $i \prec j$, and $t\left(\delta_{j, i}\right)=m$, if $j \prec i$, for any $i<j$. It is easily seen that $t$ is an abstract mono-epi type and that the mappings $t \mapsto \preceq_{t}$ and $\preceq \mapsto t_{\preceq}$ are mutually inverse. (Of course, each linear ordering $\preceq=\preceq_{t}$, interpreted as a chain of elements of $[n]$ increasing in the sense of $\prec$, can be also uniquely encoded by the sequence $(\sigma(1), \sigma(2), \ldots, \sigma(n))$, where $\sigma=\sigma_{t}$ is a permutation of $[n]$ such that $(*): i \prec j$ if and only if $\sigma^{-1}(i)<\sigma^{-1}(j)$; in particular, for any $i<j$ we have: $t\left(\delta_{j, i}\right)=e \Leftrightarrow \sigma^{-1}(i)<\sigma^{-1}(j)$, respectively, $\left.t\left(\delta_{j, i}\right)=m \Leftrightarrow \sigma^{-1}(i)>\sigma^{-1}(j)\right)$. If now an abstract mono-epi type $t$ is additionally strict then either $t\left(\delta_{n, n-1}\right)=e$ (respectively, $t\left(\delta_{2,1}\right)=m$ ) and then $\preceq$ is given by the sequence $(1,2, \ldots, n)$ (respectively, $(n, n-1, \ldots, 1)$ ), or otherwise $p-1, p+1 \prec p$, for some $p \in[n] \backslash\{1, n\}$. Then applying induction we infer that $1 \prec 2 \prec \ldots \prec p$ and $n \prec n-1 \prec \ldots \prec p$. Conversely, let $\preceq$ be a linear ordering of $[n]$, for which there exists $p \in[n]$ with the required property. Set $t:=t_{\leq}$. We can assume that $1<p<n$, since in the opposite case, for all $i<j$ we have $t\left(\delta_{j, i}\right)=e$, if $p=n$ (respectively, $t\left(\delta_{j, i}\right)=m$, if $p=1)$. Then for any $i<j<l$, the equality $t\left(\delta_{l, j}\right)=e$ implies $j<p$, and hence $t\left(\delta_{j, i}\right)=e$. Consequently, the type $t$ is always strict.

(b) Set $d(t):=\left[\sigma_{t}^{-1}(i)\right]_{i \in[n]}$. The first part of the assertion follows from the formula (*). To prove the second one fix a strict abstract mono-epi type $t$ with $p \in[n]$ as in (a) and assume that $1 \prec n$. Then there exist $i_{1}, \ldots, i_{r} \in[n]$, where $i_{1}=1, i_{2}=n, i_{r-1}=p$ and $i_{r}=p-1$, if $r$ is even (respectively, $i_{r}=p+1$, if $r$ is odd), such that $i_{1}<i_{3}<i_{5}<$ 
$\ldots \leq p+1, i_{2}>i_{4}>i_{6}>\ldots \geq p-1$ and $\sigma_{t}$ is given by the following sequence

$$
\left(i_{1}, \ldots, i_{3}-1 ; i_{2}, \ldots, i_{4}+1 ; i_{3}, \ldots, i_{5}-1 ; i_{4}, \ldots, i_{6}+1 ; \ldots, p\right) \text {. }
$$

(The cases $r$ even and $r$ odd correspond to the presence of the inequalities $p-1 \prec p+1$ and $p+1 \prec p-1$ in $\left([n], \preceq_{t}\right)$, respectively). It is not hard to see that in this situation the list of intervals

$$
I_{\left[i_{1}, i_{2}\right]}, \ldots, I_{\left[i_{3}-1, i_{2}\right]} ; I_{\left[i_{3}, i_{2}\right]}, \ldots, I_{\left[i_{3}, i_{4}+1\right]} ; I_{\left[i_{3}, i_{4}\right]}, \ldots, I_{\left[i_{5}-1, i_{4}\right]} ; \ldots ;
$$

which ends with

$$
I_{\left[i_{r-3}, i_{r-2}\right]}, \ldots, I_{\left[i_{r-1}-1, i_{r-2}\right]} ; I_{\left[i_{r-1}, i_{r-2}\right]}, \ldots, I_{\left[i_{r-1}, i_{r}+1\right]}
$$

if $r$ is even, and with

$$
I_{\left[i_{r-2}, i_{r-3}\right]}, \ldots, I_{\left[i_{r-2}, i_{r-1}+1\right]} ; I_{\left[i_{r-2}, i_{r-1}\right]}, \ldots, I_{\left[i_{r}-1, i_{r-1}\right]}
$$

if $r$ is odd, contains all, up to isomorphism, indecomposable representations in $\bmod (A)$ (intervals) which belong to $t$. We denote the set formed by all these intervals by $\mathbb{S}(t)=$ $\mathbb{S}_{A}(t)$ and call it the interval support of $t$. Simple calculation shows that always $|\mathbb{S}(t)|=n$.

Let $d=\left[d_{i}\right] \in \mathbb{N}^{[n]}$ be a dimension vector of type $t$. Then the following equalities hold:

$$
0 \leq d_{i_{1}} \leq \cdots \leq d_{i_{3}-1} \leq d_{i_{2}} \leq \ldots \leq d_{i_{4}+1} \leq d_{i_{3}} \leq \ldots \leq d_{i_{5}-1} \leq d_{i_{4}} \leq \cdots \leq d_{i_{6}+1} \leq \ldots .
$$

We set

$$
\begin{aligned}
& M(d):=\bigoplus_{i=1}^{i_{3}-i_{1}} I_{\left[\sigma(i), i_{2}\right]}^{\left(d_{\sigma(i)}-d_{\sigma(i-1)}\right)} \oplus \bigoplus_{i=i_{3}-i_{1}+1}^{i_{3}-i_{1}+i_{2}-i_{4}} I_{\left[i_{3}, \sigma(i)\right]}{ }^{\left(d_{\sigma(i)}-d_{\sigma(i-1)}\right)} \oplus \\
& \bigoplus_{i=i_{3}-i_{1}+i_{2}-i_{4}+1}^{i_{3}-i_{1}+i_{2}-i_{4}+i_{5}-i_{3}} I_{\left[\sigma(i), i_{4}\right]}\left(d_{\sigma(i)}-d_{\sigma(i-1)}\right) \oplus \ldots
\end{aligned}
$$

where $\sigma=\sigma_{t}$ and $d_{\sigma(0)}:=0$. Then $M(d)$ is a mono-epi representation of type $t$, since so do all indecomposable direct summands in the decomposition above. Moreover, it is easy to verify that $\operatorname{dim}_{k} M(d)=d$.

Let now $\bar{M}$ be a mono-epi representation with $\operatorname{dim}_{k} M=d$. Then $M$ is of type $t$; hence, so do all indecomposable direct summands in the interval decomposition of $M$. Therefore, $M$ has the shape

$$
M \cong \bigoplus_{I \in \mathbb{S}(t)} I^{a_{I}}
$$

for some $a_{I} \in \mathbb{N}$. We can determine precisely the dimension vector $\operatorname{dim}_{k}\left(\bigoplus_{I \in \mathbb{S}(t)} I^{a_{I}}\right)$, expressing it in terms of multiplicities $\left(a_{I}\right)_{I \in \mathbb{S}(t)}$. Comparing the result to $d$, we obtain an $n \times n$ system $(u)$ of linear equations over $\mathbb{Z}$ in the variables $a_{I}$, for $I \in \mathbb{S}(t)$. Once the equations are ordered according to $\prec$, whereas the variables as in the list defining $\mathbb{S}(t)$, the system $(u)$ becomes lower unitriangular. Hence, $(u)$ has precisely one (integral) solution, so the multiplicities $\left(a_{I}\right)_{I \in \mathbb{S}(t)}$ coincide with those from the formula defining $M(d)$; consequently, $M \cong M(d)$.

The dual case $n \prec 1$ requires exactly the same treatment.

(c) Let $M$ be an arbitrary mono-epi representation and $\operatorname{dim}_{k} M:=\left[d_{i}\right]_{i \in[n]}$. We show first that $\left(d_{i}\right)_{i \in[n]}$ forms an ascending-descending sequence, ${\overline{\text { i.e. } d_{1}}}_{1} \leq d_{2} \leq \ldots \leq d_{p} \geq \ldots \geq$ $d_{n-1} \geq d_{n}$, for some $p \in[n]$. Suppose this is not the case. Then there exists $1 \leq i_{1}<i_{2}<n$ 
such that $d_{i_{1}}>d_{i_{1}+1}=d_{i_{2}}<d_{i_{2}+1}$. But this is impossible, since for any $i<j<l$ we have $\delta_{l, i}=\delta_{l, j} \delta_{j, i} \in \Omega$ and the inequalities $d_{i}, d_{l} \geq d_{j}$ imply $d_{i}=d_{j}$ or $d_{l}=d_{j}$ (cf. Section 4.8). Consequently, such $p \in[n]$ always exists. If $p=n$ (respectively, $p=1$ ) then for a strict abstract mono-epi type $t$ such that $M$ belongs to $t$ we can take $t_{\preceq}$, where $\preceq$ is given by the sequence $(1, \ldots, n)$ (respectively, $(n, \ldots, 1))$. Assume now that $1<p<n$. Consider two linear orderings $\preceq_{1}$ of $[p]$ and $\preceq_{2}$ of $[n] \backslash[p-1]$, which are defined by the chains $1 \prec_{1} \ldots \prec_{1} p$ and $n \prec_{2} \ldots \prec_{2} p$, respectively. Then any integration of these two chains into the one, yields always a linear ordering $\preceq$ on $[n]$ such that the associated type $t_{\preceq}$ is strict. It is easily seen that with a slight care, applying the arguments similar to these involving the sequences $i_{1}<i_{3}<\ldots$ and $i_{2}>i_{4}>\ldots$, the integration process can be provided in a way respecting the property: $d_{i} \leq d_{j}$, if $i \prec j$, where $i<p$ and $j>p$, or $i>p$ and $j<p$. Clearly, the representation $M$ belongs to the type $t=t_{\preceq}$, for the such ordering $\preceq$.

We say that the ordering $\preceq$ of the set $[n]$, where $n \geq 2$, is almost linear if for each pair $(i, j) \neq(1, n)$ of members of $[n]$, the elements $i$ and $j$ are $\preceq$-comparable. We also set $[n]^{\prime}:=[n] \backslash\{1, n\}$ and we denote by $\left(A^{\prime}\right)^{(n)}(\cong A(n-2))$ the path algebra of the full subquiver $\left(Q^{\prime}\right)^{(n)}$ of $Q^{(n)}$ spanned by the vertex set $[n]^{\prime}$.

Proposition 5.4 Let $A=\bar{A}(n)$ and $Q=Q^{(n)}$, where $n \geq 3$.

(a) The mapping $t \mapsto \preceq=\preceq_{t}$ yields a bijection between the sets of all abstract monoepi types $t: \Omega \rightarrow\{e, m\}$ for the algebra A, where $\Omega=\Omega\left(Q, \Delta^{(n)}\right)$, and all almost linear orderings $\preceq$ of the set $Q_{0}=[n]$. In particular, via the bijection above the strict types $t$ correspond precisely to the almost linear orderings such that $1, n \prec i$, for all $i \in[n]^{\prime}$, and $2 \prec \ldots \prec p, n-1 \prec \ldots \prec p$, for some $p \in[n]^{\prime}$; so via the restriction to the orderings $\preceq_{t^{\prime}}$ of $[n]^{\prime}$, for all strict abstract mono-epi types $t^{\prime}$ for the algebra $A^{\prime}:=\left(A^{\prime}\right)^{(n)}$.

(b) For each strict abstract mono-epi type there exists a unique canonical vector $d(t)=$ $\left[d_{i}\right] \in \mathbb{N}^{Q_{0}}$ of type $t$, such that $d(t) \in[n]{ }^{Q_{0}}, d_{1}=d_{n}=1, d_{i}<d_{j}$ for all $i \prec j$, and $d_{1}+d_{n}<d_{i}$, for all $i \in[n]^{\prime}$. Moreover, for every $d=\left[d_{i}\right] \in \mathbb{N} Q_{0}$ such that $d_{1}+d_{n} \leq d_{i}$, for all $i \in[n]^{\prime}$, which belongs to $t$, there exists precisely one, up to isomorphism, mono-epi representation $M=\bar{M}(d)$ in $\bmod (A)$ with $\operatorname{dim}_{k} M=d$.

(c) Let $M$ be a mono-epi representation in $\bmod (A)$ and $\operatorname{dim}_{k} M=\left[d_{i}\right]$. If $M$ is sincere then $\operatorname{dim}_{k} M$ belongs to some strict mono-epi type $t$; $\overline{\text { moreover, }} d_{1}+d_{n} \leq d_{i}$, for all $i \in\left[\overline{n]^{\prime} . \text { If }} d_{n}=0\right.$ (respectively, $d_{1}=0$ ) then $\operatorname{dim}_{k} M$ belongs to some strict mono-epi type $t$ if and only if $d_{1} \leq d_{n-1}$ (respectively, $\overline{d_{n} \leq} d_{2}$ ); in particular, this is always the case if $d_{1}=d_{n}=0$. If $M$ is not sincere and $d_{1}=d_{n} \neq 0$ then $d_{i}=0$, for all $i \in[n]^{\prime}$.

Proof (a) Due to the proof of Lemma 5.3, to show that $\preceq=\preceq_{t}$ yields an ordering of $[n]$ we need only to verify the transitivity property for $\preceq$. (Notice that now $\Omega=\left\{\delta_{j, i}: i<\right.$ $j,(i, j) \neq(1, n)\})$. Fixing $i, j, l \in[n]$ such that $i \prec j$ and $j \prec l$, observe that if $i<j<l$ then $\delta_{l, j}, \delta_{j, i} \in \Omega$ and $t\left(\delta_{l, j}\right)=t\left(\delta_{j, i}\right)=e$, so $\delta_{l, i}=\delta_{l, j} \delta_{j, i} \in \Omega$ and $t\left(\delta_{l, i}\right)=e$; hence $i \prec l$. The case $i>j>l$ is dual to that above. In the remaining four cases we always have $\{i, l\} \neq\{1, n\}$, so automatically $\delta_{l, i} \in \Omega$, if $i<l$ (respectively, $\delta_{i, l} \in \Omega$, if $l<i$ ), and comparing to the proof of Lemma 5.3, we do not need any extra arguments. Note that by the shape of $\Omega$ the ordering $\preceq$ is almost linear. Let now $\preceq$ be an arbitrary almost linear ordering of $[n]$. We define the map $t=t_{\leq}: \Omega \rightarrow\{e, m\}$ by the same formula as in the case of $A^{(n)}$. It is well defined, since for any $i<j$ such that $(i, j) \neq(1, n)$, the elements $i$ and $j$ are 
$\preceq$-comparable. Moreover, $t$ is an abstract mono-epi type, since if we have $t\left(\delta_{l, j}\right)=$ $t\left(\delta_{j, i}\right)=e$, for $i<j<l$, then by the transitivity of $\preceq$ it follows that $i \prec l$, so $(i, l) \neq$ $(1, n), \delta_{l, j} \delta_{j, i}=\delta_{l, i} \in \Omega$ and $t\left(\delta_{l, i}\right)=e$ (similarly in the case $t\left(\delta_{l, j}\right)=t\left(\delta_{j, i}\right)=m$ ). It is clear that the mappings $t \mapsto \preceq_{t}$ and $\preceq \mapsto t_{\preceq}$ are mutually inverse, so we obtain the required bijection. Notice that each $\preceq$ as above can be uniquely encoded by the pair $\left(r, \sigma^{\prime}\right)$ (and viewed as $\left.\left(\sigma^{\prime}(2) \ldots, \sigma^{\prime}(r),\{1, n\}, \sigma^{\prime}(r+1), \ldots, \sigma^{\prime}(n-1)\right)\right)$, where $r \in[n-1]$ and $\sigma^{\prime}$ is a permutation of $[n]^{\prime}$; in particular, simply by $\sigma^{\prime}$, under the assumption that $1, n \prec i$, for all $i \in[n]^{\prime}$. Let now $t$ be a strict abstract mono-epi type for the algebra $A$ and $\preceq_{:=\iota_{t}}$. Then there exists an integer $p \in[n]^{\prime}$ such that $2 \prec \ldots \prec p$ and $n-1 \prec \ldots \prec p$, since the restriction $t^{\prime}$ of $t$ to the set $\Omega^{\prime}:=\left\{\delta_{j, i}: 2 \leq i<j \leq n-1\right\}$ is a strict abstract mono-epi type for the path algebra $A^{\prime}$, and $\preceq_{t^{\prime}}$ coincides with the restriction $\preceq_{\mid[n]^{\prime}}$ of $\preceq$ to $[n]^{\prime}$. If $p=n-1$ then the second chain trivializes and the first one has the shape $2 \prec \ldots \prec n-1$, hence we have $1 \prec 2$, since $t\left(\delta_{n-1,2}\right)=e$ implies $t\left(\delta_{2,1}\right)=e$. Moreover, we have also $n \prec i$, for every $i \in[n]^{\prime}$, since $i \prec n$ yields $t\left(\delta_{n, i}\right)=e=t\left(\delta_{i, 1}\right)$, so $\delta_{n, 1} \in \Omega$, a contradiction. Dually, we show that $n \prec n-1$ and $1 \prec i$, for every $i \in[n]^{\prime}$, if $p=2$. In the case $2<p<n-1$, applying the analogous arguments, we show first that $1 \prec 2$ and $n \prec n-1$, and next that $n \prec 2$ and $1 \prec n-1$. Since $[n]^{\prime}$ is linearly ordered by $\preceq_{t^{\prime}}$, its smallest element is either 2 or $n-1$ and we have always that $1, n \prec i$, for every $i \in[n]^{\prime}$. In this way $\preceq=\preceq_{t}$, for a strict $t$, has always the required properties. Observe that the converse implication holds by the analogous fact for the algebra $A^{\prime}$, since in our situation the equalities $t\left(\delta_{l, j}\right)=e$ and $t\left(\delta_{j, i}\right)=m$, for $i<j<l$, imply $i, l \in[n]^{\prime}$. Finally note that the assertion concerning the correspondence $t \mapsto t^{\prime}=t_{\mid \Omega^{\prime}}$ (equivalently, $\preceq \preceq_{t} \mapsto \preceq_{\mid[n]^{\prime}}$ ) follows immediately from the descriptions of the mono-epi types for $A$ in terms of permutations $\sigma^{\prime}$ of $[n]^{\prime}$, given above.

(b) To define the vector $d(t)$, for a fixed strict abstract mono-epi type $t$ for the algebra $A$, we set $d_{1}, d_{n}:=1$ and $d_{i}:=\left(\sigma^{\prime}\right)^{-1}(i)+1$, for all $i \in[n]^{\prime}$, where $\sigma^{\prime}=\sigma_{t^{\prime}}$ and $t^{\prime}$ are as above. By the previous considerations and Lemma 5.3(b) the vector $d(t)$ has the required properties. Observe also that now the set $\mathbb{S}(t)=\mathbb{S}_{A}(t)$ of all intervals in $\bmod (A)$ of type $t$ has the shape

$$
\mathbb{S}(t)=\left\{I_{[1, n-1]}, I_{[2, n]}\right\} \cup \mathbb{S}_{A^{\prime}}\left(t^{\prime}\right) .
$$

(We treat $A^{\prime}$-modules as $A$-modules by means of the standard extension by zeros embedding $\left.\bmod (A)^{\prime} \hookrightarrow \bmod (A)\right)$. It again consists of $n$ elements, since $\left|\mathbb{S}_{A^{\prime}}\left(t^{\prime}\right)\right|=n-2$. It is called the interval support of $t$.

Let $d=\left[d_{i}\right]_{i \in[n]}$ be a vector of type $t$ with the assumed properties. Then the vector $d^{\prime}:=\left(d_{i}-\left(d_{1}+d_{n}\right)\right)_{i \in[n]^{\prime}}$ belongs to the strict type $t^{\prime}$ for the algebra $A^{\prime}$. We set

$$
\bar{M}(d):=I_{[1, n-1]}^{d_{1}} \oplus I_{[2, n]}^{d_{n}} \oplus M\left(d^{\prime}\right) .
$$

$\bar{M}(d)$ is a mono-epi representation of type $t$, since so do all indecomposable direct summands in the decomposition above; moreover, $\operatorname{dim}_{k} \bar{M}(d)=d_{1} \cdot \operatorname{dim}_{k} I_{[1, n-1]}+d_{n}$. $\operatorname{dim}_{k} I_{[2, n]}+\operatorname{dim}_{k} M\left(d^{\prime}\right)=d$. If now $M$ is an arbitrary mono-epi representation with $\overline{\operatorname{dim}_{k}} M=d$ then $M$ is of type $t$ and so do all its indecomposable direct summands in the interval decomposition. Therefore, $M$ has the shape

$$
M \cong I_{[1, n-1]}^{a_{1}} \oplus I_{[2, n]}^{a_{n}} \oplus M^{\prime}
$$

where $a_{1}, a_{n} \in \mathbb{N}$ and $M^{\prime}=\bigoplus_{I \in \mathbb{S}_{A^{\prime}}\left(t^{\prime}\right)} I^{a_{I}}$, for some $a_{I} \in \mathbb{N}$. We have $M_{1}=\left(I_{[1, n-1]}{ }^{a_{1}}\right)_{1}$ and $M_{n}=\left(I_{[2, n]}{ }^{a_{n}}\right)_{n}$, so $a_{1}=d_{1}$ and $a_{n}=d_{n}$; hence, $\underline{\operatorname{dim}_{k}}\left(\bigoplus_{I \in \mathbb{S}_{A^{\prime}}\left(t^{\prime}\right)} I^{a_{I}}\right)=d^{\prime}$. Due to Lemma 5.3(b), we infer that $M^{\prime} \cong M\left(d^{\prime}\right)$, and consequently $M \cong \bar{M}(d)$. 
(c) If the mono-epi representation $M$ of $A$ is sincere then we have $d_{1}, \ldots, d_{n} \neq 0$ and $\delta_{n, 1}=\delta_{n, i} \delta_{i, 1} \in \Delta^{(n)}$, for any $i \in[n]^{\prime}$; hence, $d_{1}+d_{n} \leq d_{i}$ (see Section 4.8). On the other hand the restriction $M^{\prime}:=M_{\mid Q^{\prime}}$ of $M$ to $Q^{\prime}=\left(Q^{\prime}\right)^{(n)}$ is a mono-epi representation of the algebra $A^{\prime}$, so by Lemma 5.3(c) the vector $\operatorname{dim}_{k} M^{\prime}$ belongs to some strict mono-epi type $t^{\prime}$ for $A^{\prime}$. Let now $t$ be the unique strict abstract mono-epi type for $A$ such that $t_{\mid \Omega^{\prime}}=t^{\prime}$. It is clear that $\operatorname{dim}_{k} M$ belong to $t$, since $\preceq_{t^{\prime}}=\preceq_{\mid[n]^{\prime}}$, where $\preceq=\preceq_{t}$.

If now $M$ is not sincere with $d_{n}=0$ and $d_{1} \leq d_{n-1}$ then $d_{1} \leq d_{i}$, for every $i \in$ $[n]^{\prime}$, since the inequality $d_{i}<d_{1}\left(\leq d_{n-1}\right)$ is impossible due to the fact that $\delta_{n-1,1}=$ $\delta_{n-1, i} \delta_{i, 1} \in \Omega$ (see Section 4.8). Now constructing $t$ in the same way as above we again infer that $\operatorname{dim}_{k} M$ belongs to $t$. Note that the converse implication is obvious due to (a). The case $d_{1}=0$ is analogous.

Finally, suppose that $d_{1}, d_{n} \neq 0$ and $d_{i} \neq 0$, for some $i \in[n]^{\prime}$. Then for any $1<j<i$ (respectively $i<j<n$ ) we have $d_{j} \neq 0$ due to the arguments as above, a contradiction. In this way the proof is complete.

From now on the formulation strict mono-epi type (if there is no other concrete specification for $A$ ) will always mean that we deal with a strict abstract mono-epi type $t$ for the algebra $A$, where $A=A^{(n)}$ or $A=\bar{A}^{(n)}$, for some suitable $n \in \mathbb{N}$.

Remark 5.5 Let $i, j \in[n]$ be a pair of integers such that $1 \leq i<j \leq n$ and $2 \leq j-i<$ $n-1, Q^{\prime \prime}$ a full subqiuver of $Q=Q^{(n)}$ spanned on the vertex set $[i, j] \subseteq Q_{0}$ and $t^{\prime \prime}$ an arbitrary strict mono-epi type for the path algebra $A^{\prime \prime}:=k Q^{\prime \prime}$. Then there exists a strict extension $t$ of $t^{\prime \prime}$ to $A:=A^{(n)}$, i.e. a strict mono-epi type $t$ for $A$, with the property that for any sincere $A^{\prime \prime}$-representation of type $t^{\prime \prime}$ its extension $\tilde{M}$ to $A$ by zeros belongs to $t$. (If $1<i<j<n$, the type $t$ is not uniquely determined, since the integration of the chains $1 \prec \ldots \prec i-1$ and $n \prec \ldots j+1$ into one chain, which has to be clearly located in $\preceq=\preceq_{t}$ "below" the chain given by $\preceq_{t}$ ", can be provided in an arbitrary way). Moreover, for the algebra $A=\bar{A}^{(n)}$ the same holds always true, provided $1<i<j<n$. For $i=1$ (respectively $j=n$ ), a strict extension $t$ of $t^{\prime \prime}$ exists if and only if $j=n-1$ (respectively $i=2$ ) and additionally $t^{\prime \prime}$ is such that $1 \prec t^{\prime \prime} n-1$ (respectively $n \prec t^{\prime \prime} 2$ ).

Below we formulate some important observations which follow directly from the proofs Sections 5.3 and 5.4.

\section{Corollary 5.6 (a) If $t$ is a strict mono-epi type for A then:}

- $I_{[1, n]}$ belongs to $\mathbb{S}_{A}(t)$ and for any $I_{[i, j]}, I_{\left[i^{\prime}, j^{\prime}\right]} \in \mathbb{S}_{A}(t)$ we have $[i, j] \subseteq\left[i^{\prime}, j^{\prime}\right]$ or $\left[i^{\prime}, j^{\prime}\right] \subseteq[i, j]$, in case $A=A^{(n)}$;

- $I_{[2, n-1]}, I_{[1, n-1]}, I_{[2, n]}$ belong to $\mathbb{S}_{A}(t)$ and for any $I_{[i, j]}, I_{\left[i^{\prime}, j^{\prime}\right]} \in \mathbb{S}_{A}(t) \backslash$ $\left\{I_{[1, n-1]}, I_{[2, n]}\right\}$ we have $[i, j] \subseteq\left[i^{\prime}, j^{\prime}\right] \subseteq[2, n-1]$ or $\left[i^{\prime}, j^{\prime}\right] \subseteq[i, j] \subseteq[2, n-1]$, in case $A=\bar{A}^{(n)}$.

(b) $M$ in $\bmod (A)$ is a mono-epi representation of type $t$ if and only if $M \cong \bigoplus_{I \in \mathbb{S}_{A}(t)} I$.

It is interesting that we can also characterize strict mono-epi types in terms of sections in the Auslander-Reiten quiver $\Gamma_{A}$, where by a section we mean any connected full subquiver $\Sigma$ of $\Gamma_{A}$, which has a one element intersection with each $\tau_{A}$-orbit in $\Gamma_{A}$ (see [1]). Formulating our result we will identify intervals with their isomorphism classes. 
Theorem 5.7 The mapping $t \mapsto \mathbb{S}_{A}(t)$ yields a bijection between the sets of all strict mono-epi types for $A$ and all sections $\Sigma$ in $\Gamma_{A}$, which is determined by the equality $\Sigma_{0}=\mathbb{S}_{A}(t)$. In particular, for $A=\bar{A}^{(n)}$ the sections in $\Gamma_{A}$ correspond bijectively via the mapping $\Sigma \mapsto \Sigma \cap \Gamma_{A^{\prime}}$ to the sections in $\Gamma_{A^{\prime}}$, where $A^{\prime}=\left(A^{\prime}\right)^{(n)}$ and $\Gamma_{A^{\prime}} \hookrightarrow \Gamma_{A}$ is a canonical embedding; for any strict mono-epi type $t$ for $A$ we have $\mathbb{S}_{A}(t) \cap\left(\Gamma_{A^{\prime}}\right)_{0}=$ $\mathbb{S}_{A}(t) \backslash\left\{I_{[1, n-1]}, I_{[2, n]}\right\}=\mathbb{S}_{A^{\prime}}\left(t^{\prime}\right)$, where $t^{\prime}=t_{\mid \Omega^{\prime}}$.

Proof Since further we do not use this fact, we only outline the arguments of the proof. Assume first that $A=A^{(n)}$. We say that for a pair of intervals $I_{[i, j]}, I_{\left[i^{\prime}, j^{\prime}\right]} \in \mathbb{S}_{A}$ the condition (*) holds if : either $i^{\prime}=i+1$ and $j^{\prime}=j$ or $i^{\prime}=i$ and $j^{\prime}=j-1$. Observe that $\Sigma$ is a section in $\Gamma_{A}$ if and only if $\Sigma_{0}$ consists of intervals $I_{\left[i_{1}, j_{1}\right]}, \ldots, I_{\left[i_{n}, j_{n}\right]}$ such that (after suitable change of indexing) each pair of consecutive intervals satisfies $(*)$; in particular, we have $(* *): i_{1}=1 \leq i_{2} \leq \ldots \leq i_{n}=j_{n} \leq j_{n-1} \leq \ldots \leq j_{n}=n$, and $\operatorname{dim}_{k} I_{\left[i_{l}, j_{l}\right]}=n-l+1$, for every $l$. Now it is clear that $\mathbb{S}_{A}(t)$, for strict $t$, forms a section, since $\left|\mathbb{S}_{A}(t)\right|=n$ and for any two consecutive elements of the list in the proof of Lemma 5.3(b) the condition $(*)$ is satisfied.

Let $\Sigma$ be an arbitrary section, with $\Sigma_{0}=\left\{I_{\left[i_{l}, j_{l}\right]}: l \in[n]\right\}$ as above. We associate to $\Sigma$ the abstract mono-epi type $t=t(\Sigma)$, for $A$, by defining the permutation $\sigma$ of the elements of $[n]$. We set $\sigma(l):=i_{l}$, if $j_{l+1}=j_{l}$, and $\sigma(l):=j_{l}$, if $i_{l+1}=i_{l}$, for $l<n$; and $\sigma(n):=i_{n}=j_{n}$. Notice that $t$ determined by $\sigma$ is strict, with $p=i_{n}=j_{n}$, since by the construction we have $i_{l} \preceq i_{l^{\prime}}$ and $j_{l^{\prime}} \preceq j_{l}$, if $l<l^{\prime}$, where $\preceq=\preceq_{t}$ (cf. (**)). Now it is easy to check that $\mathbb{S}_{A}(t(\Sigma))=\Sigma_{0}$, for any section $\Sigma$, and $t(\Sigma)=t$, for any strict $t$, where $\Sigma$ is a section such that $\Sigma_{0}=\mathbb{S}_{A}(t)$.

Finally note that the case $A=\bar{A}^{(n)}$ follows easily from the previous one by the description of strict mono-epi types $t$ in Proposition 5.4(a), the formula $\mathbb{S}(t)=\left\{I_{[1, n-1]}, I_{[2, n]}\right\} \cup$ $\mathbb{S}_{A^{\prime}}\left(t^{\prime}\right)$ and the fact that $I_{[1, n-1]}, I_{[2, n]}, I_{[2, n-1]} \in \Sigma_{0}$, for any section $\Sigma$ in $\Gamma_{A}$. The remaining assertions are evident.

\section{8}

Let $\tilde{A}:=\tilde{A}(r)$, for $r \geq 2$, be the locally bounded category of the bounded quiver $\left(Q^{(\infty)}, \Delta^{(\infty)}(r)\right)$, where $\Delta^{(\infty)}(r)$ is the two-sided admissible ideal $\left\langle\alpha_{i+r} \ldots \alpha_{i+1}: i \in \mathbb{Z}\right\rangle$ in the path category $k Q^{(\infty)}$. Clearly $\tilde{A}$ is equipped with a natural action of the grup $\mathbb{Z}$, defined on vertices by the mapping $(z, i) \mapsto z+i$, which induces in a standard way the action of $\mathbb{Z}$ on $\bmod (\tilde{A})$ (cf. Section 2.1 ). For any $i \leq j$, we have a full faithful exact embedding $\mathcal{E}_{[i, j]}: \bmod \left(\tilde{A}_{[i, j]}\right) \hookrightarrow \bmod (\tilde{A})$, given by extension by zeros, where $\tilde{A}_{[i, j]}$ is a full subcategory of $\tilde{A}$ formed by the interval $[i, j]$. The embeddings $\mathcal{E}_{[i, j]}$ are compatible with the group actions. Further, we will usually identify the categories $\bmod \left(\tilde{A}_{[i, j]}\right)$ with their images via $\mathcal{E}_{[i, j]}$, spanning the subcategories which are closed under extensions. Notice that $\tilde{A}_{[1, r+1]}$ (respectively, $\tilde{A}_{[1, j]}$, for $j \leq r$ ) can be treated in a canonical way as a locally bounded category of the algebra $\bar{A}^{(r+1)}$ (respectively, $A^{(j)}$ ), so in fact $\mathcal{E}_{[1, j]}$, for $j \leq r+1$, yield canonical embeddings $\bmod \left(\bar{A}_{\tilde{A}}^{(r+1)}\right)=\bmod \left(\tilde{A}_{[1, r+1]}\right) \subseteq \bmod (\tilde{A})(\operatorname{respec}-$ tively, $\left.\bmod \left(A^{(j)}\right)=\bmod \left(\tilde{A}_{[1, j]}\right) \subseteq \bmod (\tilde{A})\right)$. Composing $\mathcal{E}_{[i, j]}$ 's with the appropriate $\mathbb{Z}$-shifts on $\bmod (\tilde{A})$, we have also that $\bmod \left(\bar{A}^{(r+1)}\right) \cong \bmod \left(\tilde{A}_{[i, j]}\right) \subseteq \bmod (\tilde{A})$, if $j-i=r$, and $\bmod \left(A^{(j-i+1)}\right) \cong \bmod \left(\tilde{A}_{[i, j]}\right) \subseteq \bmod (\tilde{A})$, if $j-i<r$. Similarly as for $r=6$, the set 
$\tilde{\mathbb{S}}=\mathbb{S}_{\tilde{A}}$ of the intervals $I=I_{[i, j]}$, for all $i \leq j$ with $j-i<r$, which are defined analogously as in Section 2.7, yields a full list of all, up to isomorphism, indecomposable objects in $\bmod (\tilde{A})$. (The intervals $I=I_{[i, j]}$ can be also regarded as $\mathbb{Z}$-shifts of the corresponding intervals from $\bmod \left(\bar{A}^{(r+1)}\right)$, or simpler, the intervals from $\bmod \left(\tilde{A}_{[i, j]}\right)$, where $\left.j-i<r\right)$. Consequently, for any $M$ in $\bmod (\tilde{A})$ we have the interval decomposition $M \cong \bigoplus_{I \in \tilde{\mathbb{S}}} I^{a_{I}}$, where $a_{I}=0$ for almost all $I$.

We say that the representation $M$ in $\bmod (\tilde{A})$ is connected, if its support is connected, i.e. $\operatorname{supp} M=[i, j]$, for some $i \leq j$.

Proposition 5.9 For any connected mono-epi representation $M$ in $\bmod \tilde{A}$ we have $\operatorname{supp} M=[i, j]$, where $j-i \leq r$; in particular, up to $\mathbb{Z}$-shift, $M$ belongs to $\bmod \left(\bar{A}^{(r+1)}\right)$.

Proof Let $M$ be a connected mono-epi representation with supp $M=[1, j]$. Suppose that $j \geq r+2$. Then the restriction $M_{\mid}:=M_{\mid[1, r+1]}$ of $M$ to $\tilde{A}_{[1, r+1]}$ is a sincere mono-epi representation of $A:=\bar{A}^{(r+1)}$, so by Proposition 5.4 it belongs to some strict abstract mono-epi type $t$ for $A$ and $M_{\mid} \cong \bigoplus_{I \in \mathbb{S}_{A}(t)} I^{a_{I}}$, with $a_{I_{[2, r+1]}}=d_{r+1}(\neq 0)$, where $\operatorname{dim}_{k} M=$ $\left(d_{i}\right)_{i \in \mathbb{Z}}$. Moreover, for any direct summand $I^{\prime}$ in the interval decomposition $M \cong \bigoplus_{I^{\prime} \in \tilde{\mathbb{S}}} I^{\prime}$ of $M$ we have $I_{r+1}^{\prime}=0$, provided $I_{r+2}^{\prime} \neq 0$, since the inequalities $I_{r+1}^{\prime}, I_{r+2}^{\prime} \neq 0$ imply $I_{\mid[1, r+1]}^{\prime} \cong I_{[2, r+1]}$, so $[2, r+2] \subseteq \operatorname{supp} I^{\prime} ;$ a contradiction. Hence, $M_{\alpha_{r+1}}: M_{r+2} \rightarrow$ $M_{r+1}$ is a zero map with $M_{r+1}, M_{r+2} \neq 0$, but this is impossible due to the mono-epi property of $M$. Consequently, we infer that supp $M \subseteq[1, r+1]$ and the assertion is proved.

Let $t$ be a strict mono-epi type for some $\tilde{A}_{[i, j]}$, where $0 \leq j-i \leq r$ (in fact, for the algebra $A$ corresponding to $\left.\tilde{A}_{[i, j]}\right)$. Furtheron each such $t$ will be called a local mono-epi type for $\tilde{A}$. We say that a connected mono-epi representation $M \operatorname{in} \bmod (\tilde{A})$ is of local mono-epi type $t$ (or belongs to $t$ ), if $\operatorname{supp} M \subseteq[i, j]$ and the restriction $M_{\mid[i, j]}$ (of $M$ to $\left.\tilde{A}_{[i, j]}\right)$ belongs to $t$.

\subsection{0}

Returning back to our basic setup we assume from now on that $r=6$, hence $\tilde{A}=\tilde{A}(6)$. In the proof of Theorem 2.8 an important role is played by two pairs of special local mono-epi types for $\tilde{A}$, whose shape is motivated by Corollary 4.14 .

Using the introduced notions, we denote by $t_{+}^{a}$ and $t_{-}^{a}$ the local mono-epi types for $\tilde{A}(=$ $\tilde{A}^{a}$ ), which are given by the almost linear orderings of the set [7], defined by the chains $(1,7 \prec) 6 \prec 2 \prec 5 \prec 3 \prec 4$ and $(1,7 \prec) 2 \prec 6 \prec 3 \prec 5 \prec 4$; moreover, by $t_{+}^{s}$ and $t_{-}^{s}$ the local mono-epi types for $\tilde{A}\left(=\tilde{A}^{s}\right)$, which are given by linear orderings of the set [5], defined by the chains $5 \prec 1 \prec 4 \prec 2 \prec 3$ and $1 \prec 5 \prec 2 \prec 4 \prec 3$, respectively. (Here, by $\tilde{A}^{a}$ and $\tilde{A}^{s}$ we mean two different copies of $\tilde{A}$ defined as in Section 2.7; namely, the full subcategories of $R$ with the object sets $\mathbb{Z}$ and $\mathbb{Z}^{\prime}$, respectively).

Proposition 5.11 The interval supports of the mono-epi types defined above look as follows:

$$
\begin{aligned}
& \mathbb{S}_{+}^{a}:=\mathbb{S}\left(t_{+}^{a}\right)=\left\{I_{[1,6]}, I_{[2,7]}, I_{[2,6]}, I_{[2,5]}, I_{[3,5]}, I_{[3,4]}, I_{[4,4]}\right\}, \\
& \mathbb{S}_{-}^{a}:=\mathbb{S}\left(t_{-}^{a}\right)=\left\{I_{[1,6]}, I_{[2,7]}, I_{[2,6]}, I_{[3,6]}, I_{[3,5]}, I_{[4,5]}, I_{[4,4]}\right\},
\end{aligned}
$$


and

$$
\begin{aligned}
& \mathbb{S}_{+}^{s}:=\mathbb{S}\left(t_{+}^{s}\right)=\left\{I_{[1,5]}, I_{[1,4]}, I_{[2,4]}, I_{[2,3]}, I_{[3,3]}\right\}, \\
& \mathbb{S}_{-}^{s}:=\mathbb{S}\left(t_{-}^{s}\right)=\left\{I_{[1,5]}, I_{[2,5]}, I_{[2,4]}, I_{[3,4]}, I_{[3,3]}\right\} .
\end{aligned}
$$

In particular, $N$ in $\bmod (\tilde{A})$, with $\operatorname{dim}_{k} N=\left[d_{z}\right]_{z \in \mathbb{Z}}$, is a mono-epi representation of local mono-epi type $t_{+}^{a}$, respectively $t_{-}^{a}$, if and only if the interval decomposition of $N$ has the shape $N \cong \bigoplus_{I \in \mathbb{S}_{+}^{a}} I$, respectively $N \cong \bigoplus_{I \in \mathbb{S}_{-}^{a}} I$; more precisely,

$$
\begin{gathered}
(*)_{+}^{a} \quad N \cong I_{[1,6]} d^{d_{1}} \oplus I_{[2,7]}^{d_{7}} \oplus I_{[2,6]}{ }^{d_{6}-d_{1}-d_{7}} \oplus I_{[2,5]} d^{d_{2}-d_{6}} \oplus I_{[3,5]}^{d_{5}-d_{2}} \\
\oplus I_{[3,4]}{ }^{d_{3}-d_{5}} \oplus I_{[4,4]}{ }^{d_{4}-d_{3}}
\end{gathered}
$$

and respectively,

$$
\begin{gathered}
(*)_{-}^{a} \quad N \cong I_{[1,6]}^{d_{1}} \oplus I_{[2,7]}^{d_{7}} \oplus I_{[2,6]}^{d_{2}-d_{1}-d_{7}} \oplus I_{[3,6]}^{d_{6}-d_{2}} \oplus I_{[3,5]}^{d_{3}-d_{6}} \\
\oplus I_{[4,5]}{ }^{d_{5}-d_{3}} \oplus I_{[4,4]}^{d_{4}-d_{5}} .
\end{gathered}
$$

Similarly, $N$ is a mono-epi representation of local mono-epi type $t_{+}^{s}$, respectively $t_{-}^{s}$, if and only if the interval decomposition of $N$ has the shape $N \cong \bigoplus_{I_{s} \in \mathbb{S}_{+}^{s}}$, respectively $N \cong \bigoplus_{I_{a} \in \mathbb{S}_{-}^{s}} I$; more precisely,

$(*)_{+}^{s} \quad N \cong I_{[1,5]}{ }^{d_{5}} \oplus I_{[1,4]}^{d_{1}-d_{5}} \oplus I_{[2,4]}^{d_{4}-d_{1}} \oplus I_{[2,3]}^{d_{2}-d_{4}} \oplus I_{[3,3]} d_{3}-d_{2}$

and respectively,

$(*)^{s}$

$$
N \cong I_{[1,5]}^{d_{1}} \oplus I_{[2,5]}^{d_{5}-d_{1}} \oplus I_{[2,4]}^{d_{2}-d_{5}} \oplus I_{[3,4]}^{d_{4}-d_{2}} \oplus I_{[3,3]}^{d_{3}-d_{4}}
$$

Proof Follows easily by Lemma 5.3 and Proposition 5.4 (see also the proofs).

\subsection{2}

We say that a module $M=\left(M^{s}, M^{a}, \varphi_{M}\right)$ in $\bmod (R)$ belongs to a mono-epi type $\left(t_{+}^{s}, t_{+}^{a}\right)$ (respectively, $\left.\left(t_{-}^{s}, t_{-}^{a}\right)\right)$ if $M^{s}$ belongs to $t_{+}^{s}$ (respectively, $\left.t_{-}^{s}\right)$ and $M^{a}$ belongs to $t_{+}^{a}$ (respectively, $\left.t_{-}^{a}\right)$. A module $M$ in $\bmod (\Lambda)$ belongs to $\left(t_{+}^{s}, t_{+}^{a}\right)$ (respectively, $\left.\left(t_{-}^{s}, t_{-}^{a}\right)\right)$ if so does the $R$-module $\hat{M}=\left(\hat{M}^{s}, \hat{M}^{a}, \varphi_{\hat{M}}\right)$ associated to $M$.

Proof of Theorem 2.8. For any quadruple $q, i, s, l$, where $q \in \overline{\mathbb{Q}}, i \in[3], s \in \mathbb{Z}_{p_{i}}$ and $l \in$ $\mathbb{N}_{1}$, we denote by $\mathcal{G}_{q, i, s, l}$ the indecomposable sheaf of quasi-length $l$ in the tube $\mathcal{T}_{\lambda_{i}}^{q}(\mathbb{X})$ with quasi-socle $\mathcal{F}_{q, i, s}$ (cf. Section 4.7; clearly $\mu\left(\mathcal{G}_{q, i, s, l}\right)=q, \rho\left(\mathcal{G}_{q, i, s, l}\right)=p_{i}$ and $\mathcal{G}_{q, i, s, 1}=$ $\left.\mathcal{F}_{q, i, s}\right)$. Moreover, we set $Y_{q, i, s, l}:=\Psi\left(\mathcal{G}_{q, i, s, l}\right)=\operatorname{Hom}\left(T, \mathcal{G}_{q, i, s, l}\right)$, if $q \geq 1$ and $\mathcal{G}_{q, i, s, l}$ is in $\operatorname{coh}_{\geq}(\mathbb{X})$, and $Y_{q, i, s, l}:=\Psi\left(\mathcal{G}_{q, i, s, l}[1]\right)=\operatorname{Ext}_{\mathbb{X}}^{1}\left(T, \mathcal{G}_{q, i, s, l}\right)$, if $q \leq 0$ and $\mathcal{G}_{q, i, s, l}$ is in coh_( $\mathbb{X}$ ) (clearly $Y_{q, i, s, 1}=X_{q, i, s}$ ).

We start by observing that $M_{i, s, l}(\gamma) \cong \hat{Y}_{q(\gamma), i, s, l}$, for all $\gamma \in \mathbb{Q}_{0}, i \in[3], s \in \mathbb{Z}_{p_{i}}$ and $l \in \mathbb{N}_{1}$ such that $(\gamma, i) \neq(0,3)$. Moreover, by Corollary 4.14, we infer that $Y_{q, i, s, l}$ 
belongs to the mono-epi type $\left(t_{+}^{s}, t_{+}^{a}\right)$, if $1 \leq q \leq \infty$ and $(q, i) \neq(\infty, 3),(1,2),(1,3)$ (equivalently, if $0 \leq \gamma:=\gamma(q) \leq 1$ and $(\gamma, i) \neq(1,3),(0,2),(0,3))$, and it belongs to $\left(t_{-}^{s}, t_{-}^{a}\right.$ ), if $q<0$ (equivalently, if $1<\gamma(q)<\infty$ ). Now the assertions follow by inserting into the decompositions from Proposition 5.11 the formulas for the dimension vectors from Corollary 2.5. More precisely, in case of the assertions (a), (c) and (d) we use $(*)_{+}^{a}$ and $(*)_{+}^{s}$, whereas in case of (b) the decompositions $(*)_{-}^{a}$ and $(*)_{-}^{s}$. Notice that the equalities in the points (iii) and (iv) of Corollary 4.14 imply that in the cases $\gamma=1$ and $\gamma=0$ (the assertions (c) and (d)) some of the members of $\mathbb{S}_{+}^{a}$ and $\mathbb{S}_{+}^{s}$ in the interval decompositions $(*)_{+}^{a}$ and $(*)_{+}^{s}$ appear always with multiplicity zero.

Remark 5.13 All the modules $M_{i, s, l}(1)$, for $i=1,2$, belong simultaneously to the type $\left(t_{+}^{s}, t_{+}^{a}\right)$ as well to $\left(t_{-}^{s}, t_{-}^{a}\right)$, so in the interval decompositions they admit also the alternative (equivalent) pairs of the exponent collections, which appear in $(*)_{-}^{a}$ and $(*)_{-}^{s}$ (cf. Corollary 4.14).

\subsection{4}

Proof of Corollary 2.9. The first assertion follows immediately by the decomposition formulas in Theorem 2.8, since for a fixed $\gamma \in \mathbb{Q}_{0}$ and $l \in \mathbb{N}_{1}$, the $R$-modules $M_{i, s, l p_{i}}(\gamma)$, for suitable $i$ and $s$, in an obvious way have the same dimension vectors, which are equal to $l \xi\left(h_{\gamma}\right)$. To prove the second one, for any $q, \lambda, l$, with $q \in \overline{\mathbb{Q}}, \lambda \in \mathbb{P}^{1}(k) \backslash\left\{\lambda_{1}, \lambda_{2}, \lambda_{3}\right\}$ and $l \in \mathbb{N}_{1}$, we denote by $\mathcal{G}_{q, \lambda, l}$ the indecomposable sheaf of quasi-length $l$ in the homogeneous tube $\mathcal{T}_{\lambda}^{q}(\mathbb{X})$ (clearly $\mu\left(\mathcal{G}_{q, \lambda, l}\right)=q$ and $\rho\left(\mathcal{G}_{q, \lambda, l}\right)=1$; moreover, we have $\left.\mathcal{G}_{q, \lambda, l} \cong \Phi_{q, \infty}\left(\mathcal{G}_{\infty, \lambda, l}\right)\right)$. Setting $Y_{q, \lambda, l}:=\Psi\left(\mathcal{G}_{q, \lambda, l}\right)=\operatorname{Hom}\left(T, \mathcal{G}_{q, \lambda, l}\right)$, if $q \geq 1$, and $Y_{q, \lambda, l}:=\Psi\left(\mathcal{G}_{q, \lambda, l}[1]\right)=\operatorname{Ext}_{\mathbb{X}}^{1}\left(T, \mathcal{G}_{q, \lambda, l}\right)$, if $q \leq 0$, we infer that $Y_{q, \lambda, l}$ belongs to the mono-epi type $\left(t_{+}^{s}, t_{+}^{a}\right)$, in the first case, and to the type $\left(t_{-}^{s}, t_{-}^{a}\right)$, in the second one (see Corollary 4.14). If now $M$ is an object (in $\tilde{\mathcal{S}}$ ) from a homogeneous tube in the family $\mathcal{T}_{\gamma}$ and has quasi-length $l$, where $\gamma \in \mathbb{Q}_{0}$ and $l \in \mathbb{N}_{1}$ are fixed, then we have $M \cong \hat{Y}_{q(\gamma), \lambda, l}$, for some $\lambda$, and the assertion follows by Proposition 5.11 and the theorem, since $\underline{\operatorname{dim}_{k}} M=l \xi\left(h_{\gamma}\right)=\underline{\operatorname{dim}_{k}} M_{1,0,2 l}(\gamma)$.

\section{Examples}

In this section we present series of examples which demonstrate in practice the application of our main results, Theorems 2.3 and 2.8, for some particular values of $\gamma$.

General Content For each of the selected indices $\gamma \in \overline{\mathbb{Q}}_{0}$, we give the continued fraction presentation $c(q)$ of the associated slope $q=q(\gamma) \in \overline{\mathbb{Q}}^{\prime}$, if $\gamma \neq 1$, and next we form the matrix $\Xi_{\gamma} \in \mathbb{M}_{10}(\mathbb{Z})$. We also provide the table $L^{q}\left(=: L_{\gamma}\right)$, which by editorial reasons has the form of a pair of tables, with two invariants concerning the quasisimple objects $M_{i, s}(\gamma)$, for $i \in[3]$ and $s \in \mathbb{Z}_{p_{i}}$. The upper rows of the tables $L^{q}$ contain the dimension vectors $\operatorname{dim}_{k} M_{i, s}(\gamma)$, the lower rows the interval multiplicity vectors $m\left(M_{i, s}(\gamma)\right)$. The columns of the tables correspond to all the consecutive (with respect 
to the coherent indexing introduced in Section 4.7) quasi-simple exceptional objects in $\tilde{\mathcal{S}}$ of index $\gamma$. More precisely, the consecutive columns of the first table contain the date for $M_{1,0}(\gamma), M_{1,1}(\gamma) ; M_{2,0}(\gamma), M_{2,1}(\gamma), M_{2,2}(\gamma)$, the columns of the second one these for $M_{3,0}(\gamma), \ldots, M_{3,5}(\gamma)$.

Format of Data The way we display the dimension vectors over $R$ in the tables is consistent with that introduced in Section 2.2, the first from the left coordinates in the rows correspond to the vertices $1^{\prime}$ and 1 in $\tilde{Q}$, respectively. As a reminder of the action of $\xi$, we use the "hat notation" for easier visualization of the recovery procedure of the dimension vectors $\operatorname{dim}_{k} X_{q, i, s}$ for quasi-simple exceptional $\Lambda$-modules $X_{q, i, s}$ with slope $q$ from $\operatorname{dim}_{k} M_{i, s}(\gamma)$ 's (see Section 4.7 for the precise definition). It is just realized by deleting the "hat coordinates" but formally one should also forget the last two zeros in the upper row of $\operatorname{dim}_{k} M_{i, s}(\gamma)$. To encode multiplicity vectors we introduce the shorten notation format which is explained by the following example: $m(M)=\frac{\left|14^{2}\right| 24|23|}{\left|16^{2}\right| 26|25| 34^{3} \mid}$, for $M=\left(M^{s}, M^{a}, \varphi_{M}\right)$ in $\bmod (R)$, means exactly that $M^{s} \cong \oplus I_{[1,4]}{ }^{2} \oplus I_{[2,4]}{ }^{1} \oplus I_{[2,3]}^{1}$ and $M^{a} \cong I_{[1,6]}^{2} \oplus I_{[2,6]}^{1} \oplus I_{[2,5]}^{1} \oplus I_{[3,4]^{3}}^{3}$.

Computation Aspects For the indices $\gamma \in \mathbb{Q}_{+}, \gamma \neq 1$, the entries of the tables $L^{q(\gamma)}$, i.e. the dimension vectors and the interval multiplicity vectors for the $M_{i, s}(\gamma)$ 's, are computed directly by the formulas from Theorems 2.3 and 2.8 , respectively. The same holds in the case $\gamma=1$, with an exception of the lower row in the second table, containing the vectors $m\left(M_{3, s}(1)\right)$. Since by Corollary 4.14 all the $M_{3, s}(1)$ 's are mono-epi representations of $R$, so even if some of them belong neither to the type $\left(t_{+}^{s}, t_{+}^{a}\right)$ nor to $\left(t_{-}^{s}, t_{-}^{a}\right)$, their interval decompositions can be immediately recovered from very simple shapes of $\operatorname{dim}_{k} M_{3, s}(1)$ 's. In the case $\gamma=0$, for the first table the situation is similar to this above. $\overline{\text { Now }}$ an exception is formed by the vectors $m\left(M_{2, s}(0)\right)$, which can be easily determined "by hand". The second table contains the invariants for the $R$-modules $M_{3, s}(0):=\hat{X}_{1,3, s}$, where $s=1,2,3,5$, from the tube $\mathcal{T}_{0}$ in $\tilde{\mathcal{S}}$, which were not formally defined in Section 2.1. The dimension vectors $\operatorname{dim}_{k} M_{3, s}(0)$ are computed by use of the standard formula involving the matrix $\Xi_{0}$ (Theorem 2.3 formally does not cover this case, so we apply $(*)$ and $(* *)$ from Section 4.7), whereas the multiplicity vectors $m\left(M_{3, s}(0)\right)$ similarly as for the previous exceptions. The "stars" in empty columns indicate the lack of data for the images of $\Psi\left(\mathcal{F}_{1,3,0}\right)$ and $\Psi\left(\mathcal{F}_{1,3,4}\right)$ by the functor $\left(^{\wedge}\right)$ (absence by obvious reasons). The case $\gamma=\infty$, added for some symmetry, concerns the data for the $R$-modules $M_{i, s}(\infty):=\hat{X}_{0, i, s}$, where $i \in[3]$ and $s \in \mathbb{Z}_{p_{i}}$, with $(i, s) \neq(3,5)$ (not considered yet). It does not yield to much new information on invariants for the exceptional quasi-simple objects from $\mathcal{D}$ (the two smaller tubes shifted by -1 coincide with those for $\gamma=0, M_{3,1}(\infty)$ does not even belong to $\tilde{\mathcal{S}}$ ). Nevertheless, it completes the description of the corresponding data for exceptional quasisimple $\Lambda$-modules in the lacking slope $q=0$. The entries of the table are computed in a very similar way as those in the case $q=1$. The analogy is almost full with only difference that the matrix $\Xi_{\infty}$ is not defined in Section 2.2, so using the standard formula we have do this now (cf. Section 4.7), and also that in the second table appears only one empty column (result of obvious absence of the image of $\Psi\left(\mathcal{F}_{0,3,5}\right)$ by $\left(^{\wedge}\right)$ ). 


\section{Tables:}

- $\gamma=1: \quad q=\infty, \quad \Xi_{\gamma}=U$

$L^{\infty}$

\begin{tabular}{|c|c||c|c|c|}
\hline$\hat{1} \hat{2} 22100$ & $\hat{0} \hat{1} 21000$ & $\hat{0} \hat{1} 11000$ & $\hat{1} \hat{1} 21100$ & $\begin{array}{c}\hat{0} \hat{1} 11000 \\
01234321\end{array}$ \\
0122210 & 012211 & 112210 & 0112110 \\
\hline$\frac{|15| 224 \mid}{|16| 27|35| 44 \mid}$ & $\frac{|24| 33 \mid}{|26| 35 \mid}$ & $\frac{|24|}{|27| 35 \mid}$ & $\frac{|1533|}{|16| 35 \mid}$ & $\frac{|24|}{|26| 44 \mid}$ \\
\hline
\end{tabular}

\begin{tabular}{|c|c|c|c|c|c|}
\hline $\begin{array}{l}0 \hat{0} 000000 \\
0011110 \\
\end{array}$ & $\begin{array}{l}\text { ô̂110000 } \\
0111111\end{array}$ & $\begin{array}{l}\text { ÔÔ111000 } \\
0011000\end{array}$ & $\begin{array}{l}\text { Ôô000000 } \\
0001100\end{array}$ & $\begin{array}{l}\text { Î1111000 } \\
1111110\end{array}$ & $\begin{array}{l}\text { Ôî11100 } \\
0111100\end{array}$ \\
\hline$\frac{1}{|36|}$ & $\frac{|23|}{|27|}$ & $\mid \frac{|34|}{|34|}$ & $\frac{11}{45 \mid}$ & $\mid \frac{|14|}{|16|}$ & $\mid \frac{|25|}{|25|}$ \\
\hline
\end{tabular}

$\left.\begin{array}{lllllllllll}1 & 3 & 2 & 2 & 0 & 0 & 1 & 0 & 1 & 2 \\ 1 & 4 & 3 & 3 & 2 & 1 & 1 & 1 & 2 & 5 \\ 1 & 5 & 3 & 3 & 2 & 1 & 2 & 1 & 2 & 9 \\ 1 & 4 & & & 1 & 1 & 1 & 1 & 2\end{array}\right]$

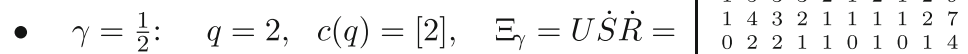

$\begin{array}{llllllllll}0 & 1 & 1 & 0 & 1 & 0 & 0 & 0 & 0 & 1\end{array}$

$\begin{array}{llllllllll}1 & 3 & 2 & 3 & 1 & 1 & 1 & 1 & 1 & 6\end{array}$

$\begin{array}{llllllllll}1 & 2 & 1 & 2 & 1 & 0 & 1 & 1 & 1 & 4\end{array}$

$\left[\begin{array}{llllllllll}1 & 1 & 0 & 1 & 0 & 0 & 0 & 0 & 1 & 1\end{array}\right]$

$L^{2}$ :

\begin{tabular}{|c|c||c|c|c|}
\hline$\hat{1} \hat{2} 32000$ & $\hat{1} \hat{3} 32100$ & $\hat{0} \hat{1} 11000$ & $\hat{1} \hat{2} 21000$ & $\hat{1} \hat{2} 32100$ \\
1244320 & 1345421 & 0123210 & 1233321 & 1233210 \\
\hline$\frac{|14| 24|33|}{|16| 25|35| 34 \mid}$ & $\frac{|15| 24|23|}{16|27| 25|35| 44 \mid}$ & $\frac{|24|}{|26| 35|44|}$ & $\frac{|14| 23 \mid}{|16| 24|35|}$ & $\frac{|15| 24|33|}{|16| 25|34|}$ \\
\hline
\end{tabular}

\begin{tabular}{|c|c|c|c|c|c|}
\hline$\hat{0} \hat{1} 10000$ & $\hat{0} \hat{1} 11000$ & $\hat{0} 0 \hat{10000}$ & $\hat{1} \hat{1} 11000$ & $\hat{0} \hat{1} 11000$ & $\hat{1} \hat{1} 11100$ \\
0111110 & 0122111 & 0011100 & 1112110 & 0111100 & 1122210 \\
\hline$\frac{|23|}{|26|}$ & $\frac{|24|}{|27| 34 \mid}$ & $\frac{|33|}{|35|}$ & $\frac{|14|}{|16| 44 \mid}$ & $\frac{|24|}{|25|}$ & $\frac{|15|}{|16| 35 \mid}$ \\
\hline
\end{tabular}

$\gamma=\frac{1}{3}: \quad q=\frac{3}{2}, \quad c(q)=[1 ; 2], \quad \Xi_{\gamma}=U \dot{S} \dot{R}^{2}=$

$\left[\begin{array}{llllllllll}0 & 1 & 1 & 1 & 0 & 0 & 1 & 0 & 1 & 3\end{array}\right.$

$\begin{array}{lllllllllll}0 & 3 & 2 & 3 & 1 & 1 & 1 & 1 & 1 & 7\end{array}$

$\begin{array}{llllllllll}0 & 5 & 3 & 4 & 2 & 2 & 2 & 1 & 2 & 11\end{array}$

$\begin{array}{llllllllll}0 & 5 & 4 & 4 & 2 & 2 & 2 & 2 & 2 & 12\end{array}$

$\begin{array}{llllllllll}0 & 4 & 3 & 3 & 1 & 2 & 1 & 1 & 2 & 9\end{array}$

$\begin{array}{llllllllll}0 & 2 & 2 & 2 & 1 & 1 & 1 & 0 & 1 & 5\end{array}$

$\begin{array}{llllllllll}0 & 0 & 0 & 1 & 0 & 1 & 0 & 0 & 0 & 1\end{array}$

$\begin{array}{llllllllll}0 & 4 & 2 & 3 & 1 & 1 & 2 & 1 & 1 & 8\end{array}$

$\begin{array}{llllllllll}0 & 2 & 1 & 2 & 1 & 0 & 1 & 1 & 1 & 5\end{array}$

$\begin{array}{llllllllll}-1 & 0 & 0 & 0 & 0 & 0 & 0 & 0 & 0 & 1\end{array}$

$L^{\frac{3}{2}}$ :

\begin{tabular}{|c|c||c|c|c|}
\hline$\hat{2} \hat{4} 43100$ & $\hat{1} \hat{3} 42000$ & $\hat{1} \hat{2} 32100$ & $\hat{1} \hat{2} 21000$ & $\hat{1} \hat{3} 32000$ \\
2467531 & 1355420 & 1244310 & 1234320 & 1344321 \\
\hline$\frac{|15| 14|24| 23 \mid}{\left|16^{2}\right| 27|25| 35|34|}$ & $\frac{|14| 24|23| 33 \mid}{|16| 26|25| 35|34|}$ & $\frac{|15| 24|33|}{|16| 25|35| 34 \mid}$ & $\frac{|14| 23 \mid}{|16| 26|35| 44 \mid}$ & $\frac{|14| 24|23|}{|16| 27|25| 34 \mid}$ \\
\hline
\end{tabular}

\begin{tabular}{|c|c|c|c|c|c|}
\hline$\hat{1} \hat{2} 21100$ & $\hat{0} \hat{1} 11000$ & $\hat{0} \hat{1} 10000$ & $\hat{1} \hat{1} 21000$ & $\hat{0} \hat{1} 11000$ & $\hat{1} \hat{1} 11000$ \\
1222210 & 0122110 & 0122211 & 1122110 & 0112100 & 1122210 \\
\hline$|15| 23 \mid$ & $\frac{|24|}{|26| 34 \mid}$ & $\frac{|23|}{|27| 35 \mid}$ & $\frac{|12| 33 \mid}{|16| 34 \mid}$ & $\frac{|24|}{|25| 44 \mid}$ & $\frac{|14|}{|16| 35 \mid}$ \\
\hline
\end{tabular}

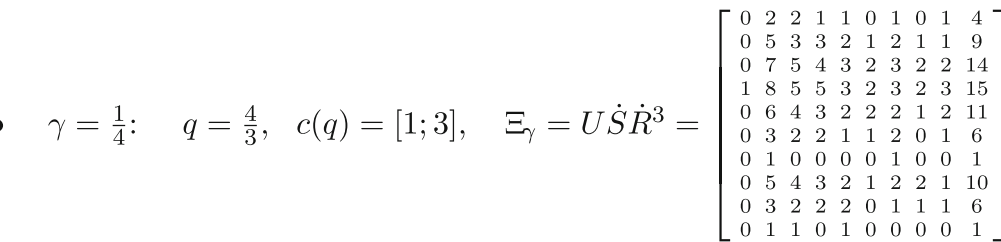

$L^{\frac{4}{3}}:$

\begin{tabular}{|c|c||c|c|c|}
\hline$\hat{2} \hat{4} 53000$ & $\hat{2} \hat{5} 53100$ & $\hat{1} \hat{3} 32000$ & $\hat{2} \hat{3} 42100$ & $\hat{1} \hat{3} 32000$ \\
2477530 & 2578631 & 1355421 & 2355420 & 1345320 \\
\hline$\frac{\left|14^{2}\right| 24|23|}{\left|16^{2}\right| 26|25| 34^{2} \mid}$ & $\frac{|15| 14|24| 23^{2} \mid}{\left|16^{2}\right| 27\left|25^{2}\right| 35|34| 44 \mid}$ & $\frac{|14| 24|23|}{|16| 27|25| 35|34|}$ & $\frac{|15| 14|23| 33 \mid}{\left|16^{2}\right| 25|35| 34 \mid}$ & $\frac{|14| 24|23|}{|16| 26|25| 34|44|}$ \\
\hline
\end{tabular}

\begin{tabular}{|c|c|c|c|c|c|}
\hline$\hat{1} \hat{2} 21000$ & $\hat{1} \hat{2} 22100$ & $\hat{0} \hat{1} 10000$ & $\hat{1} \hat{2} 21000$ & $\hat{0} \hat{1} 21000$ & $\hat{1} \hat{1} 11000$ \\
1222210 & 1233210 & 0122210 & 1233221 & 0122100 & 1123210 \\
\hline$\frac{|14| 23 \mid}{|16| 25 \mid}$ & $\frac{|15| 24 \mid}{|16| 25|34|}$ & $\frac{|23|}{|26| 35 \mid}$ & $\frac{|14| 23 \mid}{|16| 27|34|}$ & $\frac{|24| 33 \mid}{|25| 34 \mid}$ & $\frac{|14|}{|16| 35|44|}$ \\
\hline
\end{tabular}




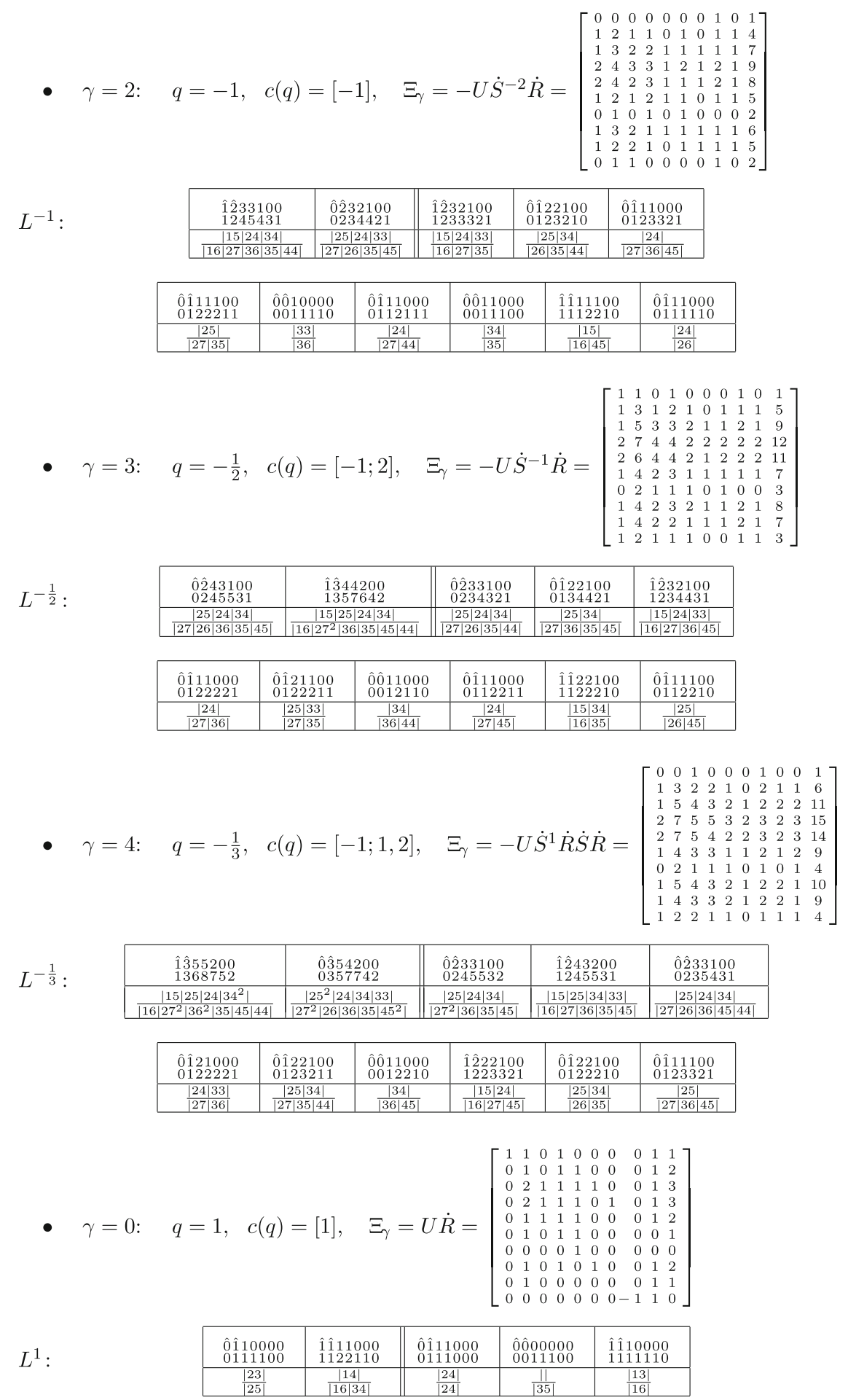




\begin{tabular}{|c|c|c|c|c|c|}
\hline$*_{0}$ & $\begin{array}{c}\hat{0} \hat{1} 00000 \\
0111111\end{array}$ & $\begin{array}{c}\hat{0} 0 \hat{1} 10000 \\
0010000\end{array}$ & $\begin{array}{c}\hat{0} \hat{0} 00000 \\
0001000\end{array}$ & $* 4$ & $\begin{array}{l}\hat{1} \hat{1} 11100 \\
1111100\end{array}$ \\
\hline$* *_{0}$ & $\frac{|22|}{|27|}$ & $\frac{|33|}{|33|}$ & $\frac{||}{|44|}$ & $* *_{4}$ & $\frac{|15|}{|15|}$ \\
\hline
\end{tabular}

\begin{tabular}{|c|c|c|c|c|c|c|c|}
\hline - $\quad \gamma=\infty$ & $q=0, \quad c(c$ & $=[0]$ & $\Xi_{\gamma}:=-U$ & $\dot{S}^{-1} \dot{R}=$ & $-\begin{array}{lllll}0 & 0 & 0 & 0 & 0 \\
1 & 0 & 0 & 1 & 0 \\
1 & 1 & 0 & 1 & 0 \\
1 & 1 & 1 & 1 & 0 \\
1 & 1 & 1 & 1 & 0 \\
1 & 1 & 1 & 1 & 0 \\
0 & 0 & 0 & 1 & 0 \\
1 & 1 & 0 & 1 & 1 \\
1 & 1 & 0 & 1 & 0 \\
0 & 0 & 0 & 0 & 0\end{array}$ & $\begin{array}{lll}0 & 0 & 1 \\
0 & 0 & 1 \\
0 & 0 & 1 \\
1 & 0 & 1 \\
0 & 1 & 1 \\
0 & 0 & 1 \\
0 & 0 & 0 \\
0 & 0 & 1 \\
1 & 0 & 1 \\
0 & 0 & 1\end{array}$ & $\begin{array}{ll}1 & 0 \\
0 & 1 \\
0 & 2 \\
0 & 3 \\
0 & 3 \\
0 & 2 \\
0 & 1 \\
0 & 2 \\
0 & 2 \\
0 & 1\end{array}$ \\
\hline \multirow[t]{4}{*}{$L^{0}$ : } & $\begin{array}{l}\hat{0} \hat{1} 11100 \\
0112211\end{array}$ & $\begin{array}{l}\hat{0} 0 \hat{011000} \\
0011110\end{array}$ & $\begin{array}{l}\hat{0} 0 \hat{011100} \\
0011100\end{array}$ & $\begin{array}{l}\hat{0} 0000000 \\
0001110\end{array}$ & $\begin{array}{l}\hat{0} \hat{1} 11000 \\
0111111\end{array}$ & & \\
\hline & $\frac{|25|}{|27| 45 \mid}$ & $\frac{|34|}{|36|}$ & $\frac{|35|}{|35|}$ & $\frac{\| 1}{|46|}$ & $\frac{|24|}{|27|}$ & & \\
\hline & $\begin{array}{l}\hat{0} 0000000 \\
0011111\end{array}$ & $\begin{array}{l}\hat{0} 0 \hat{1} 10000 \\
0000000\end{array}$ & $\begin{array}{l}\text { ôô01000 } \\
0001000 \\
\end{array}$ & $\begin{array}{l}\hat{0} 0000000 \\
0000100\end{array}$ & $\begin{array}{l}\hat{1} \hat{1} 11100 \\
1111110\end{array}$ & $*_{5}$ & \\
\hline & $\frac{11}{|37|}$ & $\frac{|33|}{\|}$ & $\frac{|| 44 \mid}{|44|}$ & $\frac{11}{|55|}$ & $\mid \frac{|15|}{|16|}$ & $* *_{5}$ & \\
\hline
\end{tabular}

Final Remark This rather natural selection of the rationals $\gamma$ provided above is not completely accidental, it is motivated by some further applications. Namely, most of the information contained in the presented tables $L^{q(\gamma)}$ play an important role in the proof of the so-called " 0 -1-property" for the class of all indecomposable objects in $\tilde{\mathcal{S}}$ lying in exceptional tubes (by the very definition this means that each such object admits an $R$ module matrix presentation involving only the coefficients 0 or 1). This result is a topic of a forthcoming publication [5].

\section{References}

1. Assem, I., Simson, D., Skowroński, A.: Elements of Representation Theory of Associative Algebras, Volume 1, Techniques of Representation Theory, London Math. Soc. Student Texts 65, Cambridge Univ. Press, Cambridge - New York (2006)

2. Birkhoff, C.: Subgroups of Abelian groups. Proc. Lond. Math. Soc. II. Ser. (1934)

3. Dowbor, P., Hübner, T.: A computer algebra approach to sheaves over weighted projective lines. In: Computational Methods for Representations of Groups and Algebras, Progress in Math. 173, Birhäuser, pp. 187-200 (1999)

4. Dowbor, P., Meltzer, H., Mróz, A.: An algorithm for the construction of exceptional modules over tubular canonical algebras. J. Algebra 323(10), 2710-2734 (2010)

5. Dowbor, P., Meltzer, H., Schmidmeier, M.: The "0-1-property" of exceptional objects for nilpotent operators of degree 6 with one invariant subspace. Preprint 57 pp., Torun (2017)

6. Gabriel, P.: Unzerlegbare Darstellungen. Manuscripta Math 6, 71-103 (1972). Berichtig Ibid 6 (309)

7. Geigle, W., Lenzing, H.: A class of weighted projective curves arising in representation theory of finite dimensional algebras. In: Singularities, representations of algebras, and vector bundles. Springer Lecture Notes in Mathematics 1273, pp. 265-297 (1987)

8. Happel, D.: Triangulated Categories in the Representation Theory of Finite Dimensional Algebras, London. Math. Soc. Lecture Notes Series 119 (1988)

9. Happel, D., Ringel, C.M.: Tilted Algebras. Trans. AMS 274(2), 399-443 (1982)

10. Kussin, D., Lenzing, H., Meltzer, H.: Nilpotent operators and weighted projective lines. J. Reine Angew. Math. 685, 33-71 (2013)

11. Kussin, D., Lenzing, H., Meltzer, H.: Triangle singularities, ADE-chains, and weighted projective lines. Adv. Math. 237, 194-251 (2013)

12. Kussin, D., Lenzing, H., Meltzer, H.: Invariant flags for nilpotent operators and weighted projective lines. Preprint, Paderborn (2017) 
13. Kussin, D., Meltzer, H.: Indecomposable modules for domestic canonical algebras. J. Pure Appl. Algebra 211(2), 471-483 (2007)

14. Lenzing, H., Meltzer, H.: Sheaves on a weighted projective line of genus one and representations of a tubular algebra. In: Representations of Algebras, Sixth International Conference, Ottawa 1992. CMS Conf. Proc. 14, pp. 317-337 (1993)

15. Lenzing, H., Meltzer, H.: Tilting sheaves and concealed-canonical algebras. In: Representation theory of algebras, Seventh International Conference, August 22-26, 1994, Cocoyoc, Mexico. Providence, RI: American Mathematical Society. CMS Conf. Proc. 18, pp. 455-473 (1996)

16. Meltzer, H.: Tubular mutations. Colloq. Math. 74(2), 267-274 (1997)

17. Meltzer, H.: Exceptional vector bundles, tilting sheaves and tilting complexes for weighted projective lines. Mem. Am. Math. Soc. 808 (2004)

18. Meltzer, H.: Exceptional modules for tubular canonical algebras. Algebr. Represent. Theory 10(5), 481497 (2007)

19. Ringel, C.M.: Tame algebras and integral quadratic forms. Springer Lecture Notes in Mathematics (1984)

20. Ringel, C.M.: Exceptional modules are tree modules. Linear Algebra Appl. 275-276, 471-493 (1998)

21. Ringel, C.M., Schmidmeier, M.: Bounded submodules of modules. J. Pure Appl. Algebra 203, 45-82 (2005)

22. Ringel, C.M., Schmidmeier, M.: Submodule categories of wild representation type. J. Pure Appl. Algebra 205, 412-422 (2006)

23. Ringel, C.M., Schmidmeier, M.: Invariant subspaces of nilpotent linear operators. I. J. Reine Angew. Math. 614, 1-52 (2008)

24. Ringel, C.M., Schmidmeier, M.: The Auslander-Reiten translation in submodule categories. Trans. Am. Math. Soc. 360(2), 691-716 (2008)

25. Simson, D.: Chain categories of modules and subprojective representations of posets over uniserial algebras. Rocky Mt. J. Math. 32(4), 1627-1650 (2002) 